\title{
Planning and Optimization of Tracking Areas for Long Term Evolution Networks
}

Sara Modarres Razavi

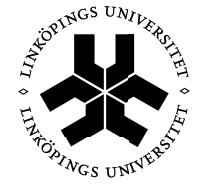

Linköping University INSTITUTE OF TECHNOLOGY

Norrköping 2014 


\section{Planning and Optimization of Tracking Areas for Long Term Evolution Networks}

Sara Modarres Razavi

Cover photo from Michael J. Photography [www.michaelslagter.com].

Linköping studies in science and technology. Dissertations, No. 1588

Copyright (C2014 Sara Modarres Razavi, unless otherwise noted.

ISBN: 978-91-7519-360-1 , ISSN: 0345-7524

Printed by LiU-Tryck, Linköping, Sweden, 2014. 


\begin{abstract}
Along with the evolution of network technologies, user's expectations on performance and mobile services are rising rapidly. To fulfill customer demands, the operators are facing a great amount of network signaling overhead in terms of Location Management (LM), which tracks and pages User Equipments (UEs) in the network. Hence, sustaining a reliable and cost-efficient LM system for future mobile broadband networks has become one of the major challenges in mobile telecommunications. Tracking Area (TA) in Long Term Evolution (LTE), is a logical grouping of cells that manages and represents the locations of UEs. This dissertation deals with planning and optimization of TAs.

TA design must be revised over time in order to adapt to changes and trends in UE location and mobility patterns. Re-optimization of a once optimized design subject to different cost budgets is one of the problems considered in this dissertation. By re-optimization, the design is successively improved by reassigning some cells to TAs other than their original ones. An algorithm based on repeated local search is developed in order to solve the resulting problem.

The next topic of research is the trade-off between the performance in terms of the total signaling overhead of the network and the reconfiguration cost. This trade-off is modeled as a bi-objective optimization problem to which the solutions are characterized by Paretooptimality. Solving the problem delivers a host of potential tradeoffs, among which the selection can be based on the preferences of a decision-maker. An integer programming model and a genetic algorithm heuristic are developed for solving the problem in large-scale networks.

In comparison to previous generations of cellular networks, LTE systems allow for a more flexible configuration of TA design by means of Tracking Area List (TAL). How to utilize this flexibility in applying TAL to large-scale networks is still an open problem. In this dissertation, three approaches for allocating and assigning TALs are presented, and their performances are compared with each other, as well as with the conventional TA scheme. Moreover, a linear programming model is developed to minimize the total signaling overhead of the network based on overlapping TALs.

In this dissertation, the problem of mitigating signaling congestion is thoroughly studied both for the specific train scenario and also for the general network topology. For each signaling congestion scenario, a related linear programming model based on minimizing the maximum signaling due to tracking area update or paging is developed. As a major advantage of the modified overlapping TAL scheme
\end{abstract}


for signaling congestion avoidance, information of individual UE mobility is not required.

Automatic reconfiguration of LM is an important element in LTE. The network continuously collects UE statistics, and the management system adapts the network configuration to changes in UE distribution and demand. In this dissertation an evaluation of dynamic configuration of TA design, including the use of overlapping TAL for congestion mitigation, is performed and compared to the static configuration by using a case study. 


\section{Populärvetenskaplig sammanfattning}

Samtidigt som mobila kommunikationstekniken utvecklas ökar användarnas förväntningar på mobila nätverkstjänster allt snabbare. För att uppfylla kundernas efterfrågan måste operatörerna hantera en stor mängd signaleringsdata kopplad till lokalisering av de mobila enheterna i nätverket. Att kunna upprätthålla pålitliga och kostnadseffektiva funktioner för hantering av de mobila terminalernas lokalisering har blivit en av de stora utmaningarna för framtidens mobila bredbandsnät. Lokaliseringsområden (Eng. Tracking Area, TA) i Long Term Evolution (LTE) är en logisk gruppering av celler som hanterar och representerar de mobila terminalernas lokalisering i nätverket. Denna avhandling handlar om planering och optimering av TA:s.

För att anpassa sig till förändringar och trender i användarnas mobilitetsmönster, måste TA-utformningen modifieras över tiden. Återoptimering av en tidigare optimerad design med olika kostnadsavvägningar är ett av de problem som behandlas i denna avhandling. Genom återoptimering kan designen successivt förbättras genom att åter tilldela vissa celler till andra TA-områden än de sina ursprungliga. För att lösa det resulterande problemet har en algoritm baserad på upprepad lokalsökning utvecklas.

Nästa problemställning som studerats handlar om prestandaavvägningar mellan den totala signaleringskostnaden i nätet och omkonfigureringskostnaden. Avvägningen är modellerad som ett optimeringsproblem med multipla målfunktioner, där Pareto-optimalitet karakteriserar lösningarna. Att lösa problemet levererar en mängd möjliga avvägningar mellan vilka valet kan avgöras baserat på beslutsfattarens preferenser. En heltalsmodell och en heuristik baserad på genetiska algoritmer är utvecklade för att lösa problemet i storskaliga nätverk.

Jämfört med tidigare generationers mobilnät, möjliggör LTE-system en mer flexibel utformning av TA-konstruktionen med hjälp av en så kallad TA-lista. Hur man kan utnyttja denna flexibilitet som TA-listor erbjuder till storskaliga nätverk är fortfarande ett öppet problem. I avhandlingen presenteras tre metoder för att konstruera och tilldela TA-listor och dess prestanda jämförs inbördes samt med en konventionell TA-konfiguration. Dessutom har en linjärprogrammeringsmodell utvecklats för att minimera den totala mängden signaleringsdata $\mathrm{i}$ nätverket baserat på överlappande TA-listor.

Avhandlingen studerar även problemet med att undvika överbelastning i nätet på grund av signaleringsdata, både för ett specifikt tåg-scenario samt för en generell nätverkstopologi. För varje scenario utvecklades en scenario-specifik linjärprogrammeringsmodell som syftar på att minimera den maximala signaleringen på grund av att anropa användare eller uppdatera lokaliseringsinformation. En stor fördel 
med det modifierade schemat för överlappande TA-listor för att hantera singaleringsöverbelastning är att schemat inte kräver information om enskilda användarens rörelser.

Automatisk omkonfigurering av funktioner för lokalisering av användare är ett viktigt inslag i LTE. Nätverket samlar kontinuerligt in användarstatistik och systemet för lokalisering av användare anpassar nätverkskonfigurationen efter förändringar i användarnas fördelning i rummet samt efterfrågan. I denna avhandling görs en utvärdering av dynamisk konfiguration av TA-listor, inklusive användning av överlappande TA-listor för att minimera risken för stockning i nätverket, och denna jämförs med den statiska konfigurationen med hjälp av en fallstudie. 


\section{Acknowledgements}

As I am not certain if the standard scheme for writing an "acknowledgement" would have the impact which I require, here I want to use a still unexplored flexibility!

I had the honor of having Prof. Di Yuan as my supervisor. It is always hard to meet the expectations of someone who has such an outstanding academic career; however, thanks to his guidance, patience and encouragement I hope I have been able to accomplish this goal. I would particularly like to thank him for sharing his experience and helping me learn how to become an independent researcher. His support was essential in my success here.

When it comes to this dissertation, I would like to acknowledge Dr. Fredrik Gunnarson and Dr. Johan Moe at Ericsson Research for our fruitful discussions, paper collaborations and for providing me with large-scale data sets. I am also grateful for the financial support I received from CENIIT, Linköping Institute of Technology, the Swedish Research Council (Vetenskapsrådet), and the MESH-WISE project. I should perhaps thank the Swedish Institute (SI) for my MSc. scholarship, without which I would never have thought of living in Scandinavia. I would especially like to thank Dr. Vangelis Angelakis for his valuable and detailed technical comments that helped me to significantly improve the quality of this work.

While thinking about all these $\mathrm{PhD}$ years, I would like to thank all my wonderful colleagues at the Department of Science and Technology, and in particular everyone at the Division of Communication and Transport Systems. My appreciation goes to Prof. Jan Lundgren and Prof. Johan M. Karlsson. I would like to say a special thank you to Lei Chen, who is the person with whom I have shared most of my memories during these years, and who has gone from being a helpful colleague and good room-mate to a true friend I can always count on. The assistance, cooperation and experience of some of my colleagues at KTS has been an enormous help in all these years, and for this I would particularly like to thank David, Anders, Joakim, Erik, Zhuangwei, and of course Viveka! There were some people who made my everyday $\mathrm{PhD}$ life one enjoyable experience, and for this I am most grateful to YuanYuan, Fahimeh, Sasan, Parisa and Ning.

Most importantly, none of this would have been possible without the love and patience of my family, who have been constant sources of love, concern, support and strength all these years. I would like to express my heart-felt gratitude to my parents, Farzaneh and Reza, and to my sister and her husband, Sonia and Navid, who are quite simply the best one can ask for. Thank you to the day-by-day improvement of 
technology, which lessens the guilt of not being beside them, where I belong and should be.

Can you imagine a person, feeling love and support at the many coffee-breaks and lunches at work? having someone to share all the difficulties of research and work at home? having someone who believes in her, even when she doubts the most? Well, I am her, and Mahziar, you are someone to whom I am not able to give a proper title: husband, friend, colleague, class-mate, all of these and more, so thanking you for your love and support properly is still hard, even in my unconventional way of acknowledgement.

Finally, I would like to dedicate this work to my two-year-old daughter, Nila, who was, of course, the reason I postponed this dissertation for one year, but her presence is my only real contribution in this world. ${ }^{1}$

Norrköping, 2014

Sara Modarres Razavi

\footnotetext{
${ }^{1}$ In the case that you find any remarks in this dissertation, now you know the reason behind it, and in terms of any lack, of course you should blame me that with all these opportunities I have fallen short in my research.
} 


\section{Abbreviations}

$\begin{array}{ll}\text { 3GPP } & \text { 3rd Generation Partnership Project } \\ \text { DCI } & \text { Downlink Control Information } \\ \text { EA } & \text { Evolutionary Algorithm } \\ \text { eNB } & \text { extended Node B } \\ \text { eNodeB } & \text { extended Node B } \\ \text { GA } & \text { Genetic Algorithm } \\ \text { GPRS } & \text { Global Packet Radio System } \\ \text { GSM } & \text { Global System for Mobile Communication, } \\ & \text { originally Group Spécial Mobile } \\ \text { HO } & \text { HandOver } \\ \text { IP } & \text { Integer Programming } \\ \text { LA } & \text { Location Area } \\ \text { LAU } & \text { Location Area Update } \\ \text { LM } & \text { Location Management } \\ \text { LP } & \text { Linear Programming } \\ \text { LS } & \text { Local Search } \\ \text { LTE } & \text { Long Term Evolution } \\ \text { MIP } & \text { Mixed Integer Programming } \\ \text { MM } & \text { Mobility Management } \\ \text { MME } & \text { Mobility Management Entity } \\ \text { MOP } & \text { Multi-objective Optimization Problem } \\ \text { MS } & \text { Mobile Station } \\ \text { MSC } & \text { Mobile Switching Center } \\ \text { MT } & \text { Mobile Terminal } \\ \text { MTAU } & \text { Maximum Tracking Area Update } \\ \text { NP } & \text { Non-deterministic Polynomial time } \\ \text { OPEX } & \text { Operating Expenditure } \\ \text { PDCCH } & \text { Physical Downlink Control Channel } \\ \text { PV } & \text { Preference Value } \\ \text { QoS } & \text { Quality of Service } \\ \text { RA } & \text { Routing Area } \\ \text { RACH } & \text { Random Access Channel } \\ \text { RAN } & \text { Radio Access Network } \\ \text { RLS } & \text { Repeated Local Search } \\ \text { RRM } & \text { Radio Resource Management } \\ \text { SAE } & \text { System Architecture Entity } \\ \text { SMS } & \text { Short Message Service } \\ \text { SON } & \text { Self Organizing Network } \\ \text { SGW } & \text { Serving Gateway } \\ \text { STA } & \text { Standard Tracking Area } \\ \text { TA } & \text { Tracking Area } \\ & \text { Tracking Area Code } \\ \text { TA } & \end{array}$




\section{Abbreviations (cont.)}

TAL Tracking Area List

TAO Tracking Area Optimization

TAP Tracking Area Planning

TAR Tracking Area Re-optimization

TAU Tracking Area Update

TPAG Total additional Paging

UE User Equipment

UMTS Universal Mobile Telecommunications System 


\section{Contents}

$\begin{array}{ll}\text { Contents } & \text { xi }\end{array}$

List of Tables $\quad$ Xv

List of Figures $\quad$ xvii

1 Introduction 1

1.1 Scope of the Dissertation . . . . . . . . . . . . . . 2

1.2 Mathematical Modeling and Optimization ........ 4

1.3 Contributions ................. 7

1.4 Publications ........................ 9

1.5 Dissertation Outline . . . . . . . . . . . . . . 10

2 Location Management in LTE $\quad 13$

2.1 Mobility Management . . . . . . . . . . . . . . . . . 13

2.2 Location Management . . . . . . . . . . . . . . . . . 15

2.3 Optimization Problems . . . . . . . . . . . . . . 22

2.4 System Model and Basic Notation . . . . . . . . . . . . . . . 24

2.5 Signaling Overhead Calculation and Unit . . . . . . . . . . 25

2.6 Description of the Lisbon Network . . . . . . . . . . . . . 26

3 Tracking Area Re-optimization 29

3.1 Problem Definition ... . . . . . . . . . . 30

3.2 Complexity and Solution Characterization . . . . . . . . . 31

3.3 A Solution Approach Based on Repeated Local Search . . . . . 33

3.4 Numerical Results . . . . . . . . . . . . . . . . . . . 37

3.5 Conclusions .................... 40

4 Trade-off in TA Reconfiguration $\quad 43$

4.1 System Model . . . . . . . . . . . . . . . . . 44

4.2 An Integer Programming Model . . . . . . . . . . . . . . . . . . . . . . . . . . 44

4.3 Dominance-based Approach . . . . . . . . . . . . . . . . 45

4.4 Heuristic Algorithm . . . . . . . . . . . . . . . . . . . . . . 47

4.5 Efficiency Improvement . . . . . . . . . . . . . 52 
4.6 Performance Evaluation $\ldots \ldots \ldots \ldots$. . . . . . . . 54

4.7 Conclusions . . . . . . . . . . . . . . . . . . . 59

5 Tracking Area List 63

5.1 Limitations of Standard TA . . . . . . . . . . . . . . . 63

5.2 Tracking Area List . . . . . . . . . . . . . . . . . . . 68

5.3 Challenges in Applying TAL . . . . . . . . . . . . . . 69

6 Applying TAL in Cellular Networks 73

6.1 Signaling Overhead Calculation for TAL . . . . . . . . . . 74

6.2 How to Design TAL? . . . . . . . . . . . . . . . . . . . . 77

6.3 Performance Evaluation of the Proposed TAL Schemes . . . . 84

6.4 Numerical Results . . . . . . . . . . . . . . . . . . . . . . . . 85

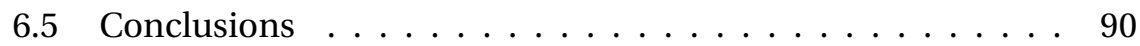

$\begin{array}{llr}7 & \text { Reducing Signaling Overhead } & 93\end{array}$

7.1 The Linear Programming Model . . . . . . . . . . . . . . . . 94

7.2 Solution Characteristics . . . . . . . . . . . . . . . 95

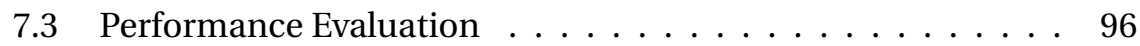

7.4 Conclusions . . . . . . . . . . . . . . . . . . 103

8 Signaling Congestion Mitigation of the Train Scenario 105

8.1 Modified Overlapping Tracking Area List . . . . . . . . . . 106

8.2 An LP Model for the Train Scenario . . . . . . . . . . . . . . . . 109

8.3 Performance Evaluation . . . . . . . . . . . . . . 111

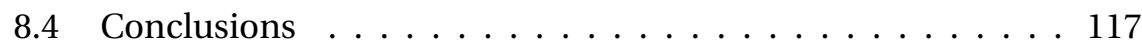

9 Signaling Congestion Mitigation $\quad 119$

9.1 An Instructive Example . . . . . . . . . . . . . . . . . . . 120

9.2 Congestion Mitigation of TAU . . . . . . . . . . . . . 122

9.3 Congestion Mitigation of Paging . . . . . . . . . . . . 123

9.4 Performance Evaluation . . . . . . . . . . . . . . . . . . . 124

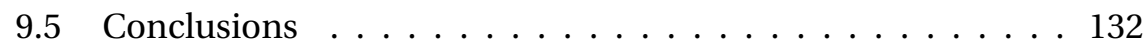

10 Study of Static and Dynamic Designs 135

10.1 SON Dynamic Framework . . . . . . . . . . . . . . . . . 136

10.2 Congestion Mitigation in SON . . . . . . . . . . . . . 137

10.3 Performance Evaluation . . . . . . . . . . . . . . 138

10.4 Conclusions . . . . . . . . . . . . . . . . . . . 147 
11 Conclusions and Future Research $\quad 149$

11.1 Conclusions . . . . . . . . . . . . . . . . . . . 149

11.2 Suggestions for Future Work . . . . . . . . . . . . . . 150

$\begin{array}{ll}\text { Bibliography } & 153\end{array}$

Appendix A Mathematical Models Notation 165

Appendix B UE-traces Scenario $\quad 169$

B.1 Generating UE-traces Scenario . . . . . . . . . . . . . . . . . . 169

B.2 Aggregating Data from UE-traces Scenario . . . . . . . . . . 170 



\section{LIST OF TABLES}

3.1 Numerical assumptions and values for performance evaluation. 39

3.2 Results of TA re-optimization. . . . . . . . . . . . . . . 40

4.1 Numerical assumptions and values for performance evaluation. 55

4.2 Minimum-overhead solutions found by the two approaches. . . . 56

6.1 Numerical assumptions and values for performance evaluation. 86

6.2 Signaling overheads of the STA design. . . . . . . . . . . . . 87

6.3 Signaling overheads of the TAL design obtained from Scheme 1. . 87

6.4 Signaling overheads of the TAL design obtained from Scheme 2. . 88

6.5 Signaling overheads of STA and TAL3 designs for 1250 UEs. . . . . 89

6.6 Signaling overheads of the TAL design obtained from Scheme 3. . 90

7.1 Numerical assumptions and values for performance evaluation. $\quad 97$

7.2 Signaling overhead comparisons of Figures 7.1 and 7.2. Figure 7.2 represents the standard TA design. . . . . . . . . . . 98

7.3 The LP model results and computation time for each group . . . 100

7.4 Load and handover data of Group 1 with their optimum TAL . . 100

7.5 Performance comparison of the two schemes in an ideal case. . . 101

8.1 Possible TA configurations, $C^{U}$ and $C^{P}$ of the ABC network. . . 107

8.2 The list of TALs for each cell in the ABC network of Figure 8.1. . . 108

8.3 Numerical assumptions and values for performance evaluation. 111

8.4 Optimal solutions for $\beta=0.3 \ldots \ldots \ldots \ldots \ldots \ldots$

8.5 Signaling overhead results for $\beta=0.3 \ldots \ldots \ldots \ldots \ldots$

9.1 Numerical assumptions and values for performance evaluation. 125

9.2 Performance comparison for TAU congestion mitigation. . . . . . 127

9.3 Performance comparison for paging congestion mitigation. . . . 130

10.1 Numerical assumptions and values for performance evaluation. 141 



\section{LIST OF FigURES}

2.1 The reporting cell scheme with bounded topology. . . . . . . . . 17

2.2 The reporting cell scheme with unbounded topology. . . . . . . 17

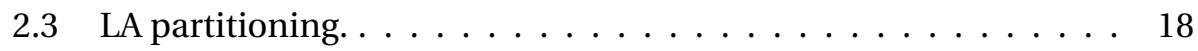

2.4 The time-based scheme. . . . . . . . . . . . . . . . . . 19

2.5 The movement-based scheme. . . . . . . . . . . . . . . . 19

2.6 The distance-based scheme. . . . . . . . . . . . . . . . 20

2.7 The shortest-distance-first scheme. . . . . . . . . . . . . . . 21

2.8 An illustration of the TAU and paging trade-off. . . . . . . . . . 22

2.9 Merging and splitting TAs. . . . . . . . . . . . . . . 23

2.10 An illustration of the Lisbon network, and the reference scenario. 27

3.1 An example of the dependency between cell moves. . . . . . . . 33

3.2 An illustration of scenario I. . . . . . . . . . . . . . . . . 38

3.3 TA design $\mathbf{t}^{0}$ (optimum of the reference scenario) . . . . . . . . 39

3.4 Re-optimized TA design for scenario I, $B^{\prime}=5 \%$. . . . . . . . . 41

4.1 An illustration of the PV definition. . . . . . . . . . . . . . 47

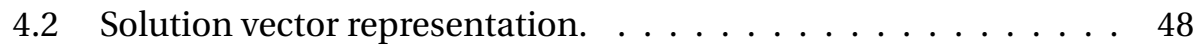

4.3 Principle design in finding Pareto-optimal configurations. . . . . 48

4.4 Applying local search to create the initial pool. . . . . . . . . . 50

4.5 The 2-point crossover method in GA. . . . . . . . . . . . 51

4.6 Quantization of the overhead and the reconfiguration cost. . . . 53

4.7 An example of the visited and PV matrices. . . . . . . . . . 53

4.8 Pareto-optimal solutions of Network 1 . . . . . . . . . . . 56

4.9 Pareto-optimal solutions of Network 2. . . . . . . . . . . . . 57

4.10 Pareto-optimal solutions of Network 3 . . . . . . . . . . . . 58

4.11 The initial TA design $\mathbf{t}^{0}$ of Network $3 \ldots \ldots \ldots$. . . . . . . 61

4.12 A Pareto-optimal solution of Network $3 \ldots \ldots \ldots 1$

5.1 (a) ping-pong effect, (b) generalized ping-pong effect. . . . . . . . 64

5.2 Example of TAU storm at the border of two TAs. . . . . . . . . 65

5.3 A three-cell network. . . . . . . . . . . . . . . . 66

5.4 An example of reducing the signaling congestion by assigning different TALs to the UEs inside the train. . . . . . . . . . . . . 69 


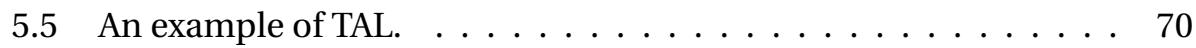

5.6 UEs are assigned to different TALs in one cell. . . . . . . . . . . 71

6.1 Parts of a network involved in one-hop estimation of the $s_{i j}(\mathbf{t}) . \quad .75$

6.2 Parts of a network involved in two-hops estimation of the $s_{i j}(\mathbf{t}) . \quad 76$

6.3 An example of the dependency between elements of $\mathbf{S}(\mathbf{t}) \ldots \ldots$

6.4 An example of how to collect part of UE traces. . . . . . . . . . 83

7.1 An illustration of the site groupings for the overlapping TAL scheme. 98

7.2 An illustration of the optimum standard TA design. . . . . . . . . 99

7.3 Evaluating the overlapping TAL scheme by Method I. . . . . . . . 102

7.4 Evaluating the overlapping TAL scheme by Method II. . . . . . . . 103

7.5 Comparison of the overlapping TAL overheads by Method I and II. 104

8.1 Three cells along a train path. . . . . . . . . . . . 107

8.2 Definition of variables for the train scenario. . . . . . . . . . 110

8.3 Evaluating the two schemes for the baseline scenario. . . . . . . . 112

8.4 Congestion Scenario 1. . . . . . . . . . . . . . . . . . 113

8.5 Evaluating the two schemes for Scenario 1. . . . . . . . . . . 113

8.6 Congestion Scenario 2. . . . . . . . . . . . . . . . . . . 115

8.7 Evaluating the two schemes for Scenarios 1 and 2. . . . . . . 115

9.1 A small network with four TAs. . . . . . . . . . . . . . . . . 121

9.2 The underlying TA design. . . . . . . . . . . . . . . . . 127

9.3 Evaluating the TAU congestion mitigation. . . . . . . . . . . . 128

9.4 The standard TA design for a chosen maximum TAU. . . . . . . . 129

9.5 The overlapping TAL design for a chosen maximum TAU. . . . . . 130

9.6 Evaluating the paging congestion mitigation. . . . . . . . . . 131

10.1 An illustration of the number of mobility in the Lisbon network on a Monday dawn (3:00-4:00). . . . . . . . . . . . . . 139

10.2 An illustration of the number of mobility in the Lisbon network on a Friday evening (17:00-18:00) . . . . . . . . . . . . 140

10.3 The total signaling overhead of the static and SON standard TA schemes. . . . . . . . . . . . . . . . . . . . 141

10.4 The TAU signaling overhead of the static and SON standard TA schemes. . . . . . . . . . . . . . . . . . . . . . 142

10.5 The maximum TAU signaling congestion of the static and SON standard TA schemes. . . . . . . . . . . . . . . . . . . . . 143 
10.6 The average TAU signaling congestion of the static and SON standard TA schemes. . . . . . . . . . . . . . . . . . . . . . . 144

10.7 The TAU signaling congestion mitigation by the overlapping TAL scheme $(\beta=0.1) \ldots \ldots \ldots \ldots \ldots \ldots \ldots \ldots$

10.8 Total additional paging $(\beta=0.1) . \ldots \ldots \ldots \ldots \ldots \ldots$

10.9 The TAU signaling congestion mitigation by the overlapping TAL scheme $(\beta=0.05) \ldots \ldots \ldots \ldots \ldots \ldots \ldots$

10.10 Total additional paging $(\beta=0.05) \ldots \ldots \ldots \ldots \ldots \ldots$

B.1 An example of a row in the scenario matrix. . . . . . . . . . 170 



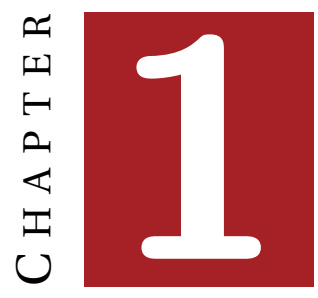

\section{INTRODUCTION}

The enormous competition in the telecommunications market results in the necessity of optimized and cost-efficient networks for the operators and service providers. Long Term Evolution (LTE) was initiated to bring mobile broadband via new technology, applications and services to the wireless cellular networks. This results in new architectures and configurations.

Managing mobility has become a prerequisite for any wireless network and telecommunication service. Mobility management (MM) aims to track user equipment (UE) and to allow calls, SMS and other mobile phone services to be delivered to UE. Any mobility protocol involves the solving of two separate problems. One is the location management (LM), sometimes called reachability, that keeps track of the positions of a UE in the mobile network. The other is handover management, sometimes called session continuity, which makes it possible for a UE to continue its sessions while moving to another cell and changing access point. The focus of this dissertation is on location management issues.

Tracing the mobile UE cost-efficiently is one of the major challenges of the study of location management in wireless cellular networks. The Tracking Area (TA) is a logical grouping of cells in LTE networks. TA manages and represents the location of UE. The concept of TA is counterpart to the concepts of Location Area (LA) in GSM and Routing Area (RA) in GPRS and UMTS networks [1]. One well-known performance consideration is the signaling overhead of tracking area update (TAU) versus that of paging. This dissertation deals with the planning and optimization of tracking area configuration in LTE networks.

There are two new concepts that have been standardized by the 3rd Generation Partnership Project (3GPP) in LTE networks, that need to be more 
thoroughly explored and investigated with the objective of improving the network performance. One is the TA list (TAL) [2] in location management, which gives more flexibility to the network's configuration and has the potential of improving the network performance in terms of location management. The other concept is that of self-organizing networks (SON) [6, 9], which aim to reduce the cost of network set-up and the operating expenditure (OPEX) cost by continuously optimizing radio resource management (RRM) parameters for coverage, throughput, load balancing, and other performance targets. TA optimization is one of the target case studies for SON, as the TA design must be revised over time, due to changes in user distribution and mobility patterns [10]. Both of these concepts are explored and studied in this dissertation with the main aim of reducing the OPEX cost and improving the overall performance of location management in LTE.

\subsection{Scope of the Dissertation}

The dissertation aims to address some TA planning and optimization problems and concepts in the location management of LTE networks. In designing the layout of TAs, signaling overheads generated by the TAU and paging signaling pose a major concern, which lead to two types of optimization problems. The first is to minimize the sum (or equivalently the average) of TAU and paging signaling overheads in the network. The second takes a load balancing perspective, and has the performance target of reducing congestion, i.e., avoiding heavy amounts of signaling overhead in any part of the network caused by many UEs behaving in a similar manner, e.g., the massive and simultaneous UE mobility in a train scenario. Both sets of problems have been extensively studied in this dissertation.

In the standard scheme of TAU and paging, the Mobility Management Entity (MME) records the TA in which the UE is registered. When a UE moves to a new TA, there will be a TAU signaling overhead. The paging signaling overhead happens when the UE is being called. In order to place the call to the UE, MME broadcasts a paging message in all cells of the UE's registered TA.

Consider a TA design that is optimized for a network in the planning phase. As UE distribution and mobility patterns change over time, the optimized TA configuration will no longer perform satisfactorily. Therefore a TA reconfiguration is required to reduce the signaling overhead. This dissertation suggests a re-optimization approach for revising a given TA design. The approach is justified by the fact that once a TA design is in use, it is not feasi- 
ble to deploy a green-field design that significantly differs from the current available one.

Reconfiguring TA, such as moving a cell from its original TA to another, usually requires restarting the cell, and consequently results in service interruption. Thus, there is a trade-off between approaching minimum signaling overhead and the cost resulting from reconfiguration. In this study, a bi-objective optimization framework is proposed to solve the TA reconfiguration problem.

The Tracking Area List (TAL) is a scheme that was introduced in 3GPP Release 8 [2]. In this scheme, instead of assigning one TA to each UE, a UE can have a list of TAs. The UE receives a TA list from a cell, and keeps this list until it moves to a cell that is not included in the list. In LTE standards, a cell is also able to give different lists to different UEs. The UE location is known in the MME to at least the accuracy of the TAL allocated to that UE. If the information about each individual UE's movement was available to the network, then designing an optimum TAL, which could essentially result in the elimination of TAU signaling overhead, would become trivial. However, this information is virtually impossible to obtain. The dissertation presents solution approaches and novel analyses to shed light on TAL assignment.

One of the advantages of TAL that is explained in this dissertation is signaling congestion mitigation. The rationale for avoiding signaling congestion is to ensure no significant degradation in the quality of service which occur due to resource exhaustion for tracking or paging UE. There are several types of mobility patterns that may cause TAU congestion. For example, in densely-populated cities, there is a drastic difference in the day and night mobility behavior of UE. A very large number of UEs moving concurrently into a central area (typically on a weekday early morning) may generate excessive signaling around the center $[3,74,78]$. Conversely, heavy paging may occur as a result of massive and close-to-static UEs simultaneously located at some hotspot (e.g., a large stadium).

In LTE, there is a possibility to change the TAL assigned to each cell in short time intervals without any cost of service interruption. This is the main reason for exploring the SON dynamic framework in LTE systems. In SON, an automated and efficient deployment of updated configurations is possible. For a stable optimization, a global view of the UEs' movements and call arrival rate statistics is a requirement. In a static TA, the average of this statistic is used for the TA design. However, the dynamic framework which is presented in the dissertation incorporates both time of day and day of the week data patterns into the design, which can further strengthen the perfor- 
mance of the network over the static TA.

\subsection{Mathematical Modeling and Optimization}

Mathematical modeling characterizes the planning process such that it allocates resources in the best possible way. Optimization is the science of making the best decision, which in engineering tasks means minimizing costs and maximizing profits. Mathematical modeling and optimization methods are the main tools used for approaching the TA planning and optimization problems described in this dissertation. There is an extensive literature on optimization theory and applications (interested readers are referred to $[57,72,77,113])$. Here a brief introduction to the mathematical formulations and heuristic solution algorithms which are used in this study, are presented.

A general optimization problem can be formulated as follows;

$$
\begin{aligned}
& \min f(\mathbf{x}) \\
& \text { s. t. } \mathbf{x} \in X .
\end{aligned}
$$

where $f(\mathbf{x})$ is an objective function which depends on decision variables $\mathbf{x}$. The set $X$ defines the feasible solutions to the problem. Usually $X$ is expressed by constraints. Different optimization problem classes are obtained as a result of the specifications of $\mathbf{x}$ and set $X$. Below some of these classes are presented further.

\subsubsection{Linear Programming}

When the constraint functions defining $X$ are linear and the variables are continuous, the formulation is a linear programming problem (LP) $[26,37]$. An LP can be written in the following general form:

$$
\begin{aligned}
& \min \sum_{j=1}^{n} c_{j} x_{j} \\
& \text { s. t. } \sum_{j=1}^{n} a_{i j} x_{j} \geq b_{i}, \quad i=1, . ., m \\
& x_{j} \geq 0, \quad j=1, \ldots, n \text {. }
\end{aligned}
$$


Converting the above formulation to a maximization linear program is straightforward. LP problems can be solved to optimality by the simplex method [41], which finds the optimal solution by moving along the edges of the polyhedron from one vertex to another adjacent vertex with the goal of not worsening the objective function. The logic behind the method is the fact the optimum occurs either at a vertex of the polyhedron or on its edge or facet. Another method for solving LP models is the interior-point algorithm [63], which reaches an optimal solution by moving through the interior of the feasible region.

\subsubsection{Integer Programming and Combinatorial Optimization}

Integer programming (IP) and mixed-integer programming (MIP) are extensions of LP where a subset of the variables (at least one) are defined as integer variables. An MIP can be written in the following general form:

$$
\begin{aligned}
& \min \sum_{j=1}^{m} c_{j} x_{j}+\sum_{k=1}^{n} h_{k} y_{k} \\
& \text { s.t. } \sum_{j=1}^{m} a_{i j} x_{j}+\sum_{k=1}^{n} g_{i k} y_{k} \geq b_{i}, \quad i=1, . ., p \\
& x_{j} \in Z^{+}, \quad j=1, . ., m \\
& y_{k} \in R^{+}, \quad k=1, \ldots, n \text {. }
\end{aligned}
$$

In practice, IP and MIP formulations are frequently the candidates for modeling many engineering problems. This is first because the integer variables can represent discrete quantities, and second, the decisions which can only take zero and one are integer variables. The set of problems which involves finding an optimal solution from a finite set of solutions is referred to as the combinatorial optimization. These problems are often formulated as an IP. For a comprehensive study of different examples of combinatorial optimization and integer programming, readers are referred to $[38,62,90$, $103,115]$.

\subsubsection{Multi-objective Optimization}

Real problems in the industry are usually large complex optimization problems involving many criteria, and they are seldom mono-objective. The optimal solution to a multi-objective optimization problem (MOP) is not a 
single solution, but a set of solutions defined as Pareto optimal solutions. The generation of Pareto-optimal or non-dominated solutions is the primary goal of solving MOP problems. A solution is called Pareto-optimal if it is not possible to improve a given objective without deteriorating at least one other objective [108]. Clearly it does not make sense to choose a solution that is not Pareto-optimal. A large number of references for MOP are available in the literature $[104,105,108]$.

\subsubsection{NP-hardness}

In computational complexity theory, NP-hardness refers to a class of problems for which there so far exists no polynomial algorithm that can guarantee optimality. A problem is considered not hard when it can be solved in polynomial time, in this case with using efficient algorithms and powerful computers, it is possible to solve that problem to optimality. NP-complete problems are a subset of $N P$ class problems. Any $N P$-complete problem can be transformed to any other $N P$-complete problem with a polynomial time technique. Until now no polynomial algorithm exists which solves any of the $N P$-complete problems. If one founds an efficient technique for solving one $N P$-complete problem, then all the problems in this class could be solved efficiently by using the same technique. A problem is NP-hard if and only if there exists an $N P$-complete problem that is reducible to that problem in a polynomial time. One main strategy for proving the $N P$-hardness of a specific problem is to transform any instance of an NP-complete problem to a defined instance of that specified problem. For more information about $N P$ class problems, readers are referred to $[50,58]$.

\subsubsection{Optimization Methods}

Depending on its complexity, a problem may be solved either by an exact or an approximate method. Exact methods, such as dynamic programming [25], branch-and-X family algorithms (branch-and-bound [67], branch-andcut [77], branch-and-price [21]), etc. obtain solutions and guarantee their optimality. Some of these exact algorithms are integrated into optimization solvers such as CPLEX [60] and GUROBI [56]. Unless $P=N P$ (which is so far unknown), the exact algorithms are non-polynomial in time for NPcomplete problems.

Heuristics are the most common methodology applied for NP-hard problems. Heuristic techniques are the approximate rule-of-thumb techniques 
where optimality cannot be assured. They are usually applied for solving optimization problems which take enormous amounts of computational time. Some examples of heuristic approaches are genetic algorithms (GA) [59, 65, 98], simulated annealing [11, 64], Tabu search [51, 52, 53], local search (LS) [12], etc. In practice, the choice of heuristic depends on the structure of the problem, and it is not a trivial task. Below the two heuristic approaches which have been used in this dissertation are explained.

\subsubsection{Local Search (LS)}

The LS algorithms move from one feasible solution to another in the search space by applying local changes, until a local optimum is found or some stopping criterion is met. LS finds the best solution within a neighborhood, and unless the neighboring solutions cover the entire solution space, the final solution is often a local optimum. Algorithms such as simulated annealing and Tabu search, are enhanced local search mechanisms providing techniques for escaping from local optima. As LS tends to get stuck in suboptimal regions, the repeated local search (RLS) algorithm is used in this dissertation. In RLS, the local search is restarted in some ways by multiple initial solutions, and hence improves the performance of LS.

\subsubsection{Genetic Algorithm (GA)}

GAs are a popular class of evolutionary algorithms (EAs) which use a probabilistic selection that mimics the adaptive processes of natural systems such as the natural immune system $[54,59,108]$. The rationale behind a GA algorithm is the fact that strong species tend to survive and adapt, while the weak species tend to "die". A GA iteratively applies operations such as crossover and mutation to a population of possible solutions starting with an initial set. A mutation operation randomly perturbs a part of a candidate solution to promote diversity. There are methods for selecting the candidate solutions which are suitable to allow into the crossover and mutation operations.

\subsection{Contributions}

The main contributions of the dissertation can be summarized as follows. The corresponding chapter where the contribution has been presented is given inside the parentheses. 
- Formulating the TA re-optimization problem as an IP model. The formulation aims to optimize the trade-off between TAU and paging signaling overheads in a network with a budget constraint on the amount of reconfiguration (Chapter 3).

- Developing a heuristic approach for solving the above trade-off problem close to optimality, by using a repeated local search algorithm (Chapter 3).

- Developing two solution approaches to deliver the Pareto-optimal solutions of a bi-objective optimization problem. The computational results of both solution approaches are given for several real-life, largescale networks (Chapter 4).

- Exploiting the concept of TAL in order to improve the performance of LTE networks and presenting three schemes to design TAL for a largescale network (Chapters 5 and 6).

- Exploring the challenges in the TAL scheme and suggesting a formulation to calculate the signaling overhead in TAL. The performance of the three suggested schemes for assigning and allocating TALs are compared with this signaling overhead formulation for a large-scale network (Chapters 5 and 6).

- Formulating the TAL allocation problem with the objective of reducing the total signaling overhead as an LP model. The formulation is able to explore the potential of the list concept to a wider extent compared to the three previous proposed approaches (Chapter 7).

- Presenting an optimization framework which overcomes the uncertainty in modeling UE mobility by applying modified overlapping TALs. A main advantage of the framework is that no mobility model or detailed UE mobility information is required (Chapters 8 and 9).

- Formulating the mitigation of signaling congestion problem as two separate LP models. The formulations aim to minimize the maximum TAU or paging in the network by allowing additional amount of increase on the other (Chapters 8 and 9).

- Presenting a SON dynamic framework which smoothly reduces the impact of the older data sets. The performance of the standard TA 
scheme is evaluated for static and dynamic frameworks. The problem of congestion mitigation is explored in a dynamic framework of a large-scale network for a one-week data scenario (Chapter 10).

\subsection{Publications}

This dissertation is based on the author's material and studies which have been presented and previously appeared in the following journal publications:

- $\quad$ S. Modarres Razavi, D. Yuan, F. Gunnarsson, and J. Moe. Performance and cost trade-off in tracking area reconfiguration: A pareto-optimization approach. Computer Networks, Elsevier, 56:157-168, 2012.

- $\quad$ S. Modarres Razavi and D. Yuan. Mitigating signaling congestion in LTE location management by overlapping tracking area lists. Computer Communications, Elsevier, 35:22272235, 2012.

- $\quad$ S. Modarres Razavi, D. Yuan, F. Gunnarsson and J. Moe. On dynamic congestion mitigation by overlapping tracking area lists, submitted to Journal of Network and Computer Applications, Elsevier, 2013.

Parts of the dissertation have been published and presented at the following conferences:

- S. Modarres Razavi and D. Yuan. Performance improvement of LTE tracking area design: A re-optimization approach. In Proc. of the 6th ACM International Workshop on Mobility Management and Wireless Access (MobiWac '08), pages 77-84, 2008.

- $\quad$ S. Modarres Razavi, D. Yuan, F. Gunnarsson and J. Moe. Optimizing the trade-off between signaling and reconfiguration: A novel bi-criteria solution approach for revising tracking area design. In Proc. of IEEE Vehicular Technology Conference (VTC'09-Spring), 2009. 
- $\quad$ S. Modarres Razavi, D. Yuan, F. Gunnarsson and J. Moe. Exploiting tracking area list for improving signaling overhead in LTE. In Proc. of IEEE Vehicular Technology Conference (VTC'10-Spring), 2010.

- $\quad$ S. Modarres Razavi, D. Yuan, F. Gunnarsson and J. Moe. Dynamic tracking area list configuration and performance evaluation in LTE. In Proc. of Global Communications (GLOBECOM) Workshops, 2010.

- $\quad$ S. Modarres Razavi and D. Yuan. Mitigating mobility signaling congestion in LTE by overlapping tracking area lists. In Proc. of the 14th ACM International Conference on Modeling, Analysis and Simulation of Wireless and Mobile Systems (MSWiM'11), pages 285-292, 2011.

- $\quad$ S. Modarres Razavi and D. Yuan. A dynamic overlapping tracking area list model for mitigating signaling congestion. Poster presentation in Swedish Communication Technologies Workshop (Swe-CTW'13), 2013.

- $\quad$ S. Modarres Razavi and D. Yuan. Reducing signaling overhead by overlapping tracking area list in LTE. Accepted in the 7th IFIP Wireless and Mobile Networking Conference (WMNC'14), 2014.

The dissertation is a development of the author's Licentiate thesis.

- $\quad$ S. Modarres Razavi. Tracking Area Planning in Cellular Networks. Licentiate Thesis No. 1473, Linköping Studies in Science and Technology, Linköping University, 2011.

\subsection{Dissertation Outline}

This dissertation is written as a monograph in order to provide the opportunity of presenting the ideas and the work without the restrictions imposed by the publications in terms of templates and page limitations, and also to avoid the overlap in the papers. The rest of the dissertation is organized as follows. 
Chapter 2 presents a brief review on the previous studies done in location management, and then explains the standard TA scheme. In this chapter, the basic notation, the signaling overhead formulation and the description of the Lisbon network used throughout this dissertation are presented.

Chapter 3 presents the re-optimization approach for revising the TA design. The service interruption caused by TA reconfiguration is explicitly taken into account. The complexity and solution characterization of the resulting optimization problem are investigated. In this chapter, an algorithm which is able to deliver high-quality solutions in short computing time is developed.

Chapter 4 proposes the bi-objective optimization framework for solving the trade-off between the signaling overhead and the cost of TA reconfiguration. To obtain the Pareto-optimal solutions, two approaches have been suggested and compared. For performance evaluation, the approaches have been applied to several real-life large-scale networks.

In Chapter 5, the reader is introduced to the concept of Tracking Area List in LTE systems. This chapter illustrates the potential of TAL by clarifying the limitations of the standard TA scheme. The challenge in applying TAL to a large-scale network is explained.

Chapter 6 proposes a formula for calculating the signaling overhead in TAL. The chapter presents three schemes to design TAL with the available data at hand, and discusses the pros and cons of each scheme. For performance evaluation, a method is presented to calculate the exact total signaling overhead. A thorough study of the numerical results is presented to compare the standard TA scheme with the three suggested TAL schemes.

Chapter 7 presents an optimization model which can solve the problem of minimizing the total signaling overhead with the TAL concept. The solution characterization of the resulting optimization problem is investigated. The standard limitation of the number of TAs in a TAL is explicitly taken into account. Two methods are presented for comparing the TAL design solution obtained by the model with the standard TA scheme on a large-scale network.

Chapter 8 considers the signaling congestion problem of the train scenario. A model which is based on overlapping TALs to mitigate the TAU signaling congestion by allowing a limited additional increase in the overall paging, is presented. The model used in this chapter is independent of each UE's individual movement. The performance of the model has been compared with the standard TA scheme for different congestion scenarios.

Chapter 9 generalizes the overlapping TAL model presented in Chapter 8 
for both TAU and paging signaling congestion mitigation of a general topology. The models have been applied to a large-scale network and compared to the standard TA scheme. As the models are insensitive to individual UE movements, for performance evaluation, no assumption on UE mobility or other network parameters is required.

In Chapter 10, a dynamic framework suitable for self-organizing networks is proposed. A comprehensive study on the performance of static and dynamic standard TA scheme is presented. The overlapping TAL model is applied to the dynamic framework in order to dynamically mitigate the TAU signaling congestion in a large-scale network.

In Chapter 11, the author draws some conclusions and gives an overview of possible extensions to the dissertation work.

To provide more clarification, the dissertation is followed by two appendices.

Appendix A is a definition list of all parameters, sets and variables used in the different chapters of the dissertation.

In Appendix B, the reader is presented with an approach for generating UE-traces scenarios and a method that can be used to aggregate the data from them. The performance evaluation of the TAL schemes presented in Chapter 6 is applied on these UE-traces scenarios. 


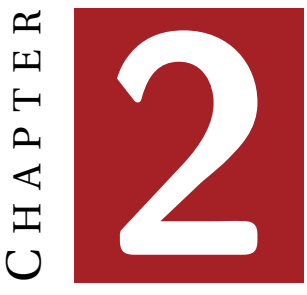

\section{Location Management in LTE}

Location management is one of the fundamental problems in providing the mobility feature for cellular networks. It deals with how to track user equipment (UE) that is on the move. All the technical terms and concepts in this dissertation are based on Long Term Evolution (LTE) systems. The chapter provides a technical background to the Tracking Area Update (TAU) and paging function, but for more information and details, readers are referred to 3GPP documentation on these topics [7, 8]. Another purpose of this chapter is to survey the research carried out on the topic of location management in mobile cellular networks. It also presents some background and information about basic materials for tracking area planning (TAP). Moreover, it also presents the signaling overhead formulation under the standard scheme and the description of the Lisbon network, which are central to the dissertation.

\subsection{Mobility Management}

One of the most essential factors when considering a successful network deployment is mobility management which provides and supports mobility and handover procedures. Mobility management is divided into two main areas: handover management and location management. In some literature, the term mobility management is used instead of location management [69, 71]. However, here the more common classification, which considers location management as an element of mobility management, is used. Although handover management is not the subject of this dissertation, I would like to refer the readers to the impressive number of surveys 
on mobility management systems for handover schemes [14, 31, 102, 119].

Mobility Management Entity (MME) is a part of the System Architecture Entity (SAE), that is the core network architecture of 3GPP's LTE wireless communication standards. The MME supports the most relevant control plane functions related to mobility [19], which are as follows:

- authentication of the UE as it accesses the system,

- managing the UE in the idle mode,

- supervising handovers between different base stations,

- establishing bearers as required for voice and Internet connectivity in a mobile context,

- generating billing information,

- implementing so-called lawful interception policies,

- oversees a large number of features defined by 3GPP specifications [2].

Among the functions related to MME, this study centres on the second task, which is managing the UE in the idle mode.

\subsubsection{User Equipment States in Mobility Management}

Any device used directly by an end-user to communicate through the cellular network is called User Equipment (UE) in LTE. Almost the same concept was previously called Mobile Station (MS) or Mobile Terminal (MT) in previous generations of cellular networks. UE can be a hand-held telephone, a smart phone, a laptop computer or any other device equipped with mobilebroadband adaptor. From a mobility perspective, the UE can be in one of the three following states.

- LTE Active: The network knows the cell to which the UE belongs, and the UE can transmit and receive data from the network. From the mobility management viewpoint, the UE may perform handover, but not location management processes.

- LTE Idle: The network knows the location of the UE at the granularity of a group of cells (forming a Tracking Area, TA). In the idle mode, the $\mathrm{UE}$ is in power-conservation mode and does not inform the network of each cell change.

- LTE Detached: In this mode, the UE is either powered off, or it is in the transitory state in which it is in the process of searching and registering to the network. 
The UE will frequently be in the LTE-Idle state, and the MME knows the TA in which the UE was last registered. Usually, the only realistic data available from a cellular network are the cell load and cell handovers. Cell load and handover represent active UEs. Cell load and handover statistics can be a good estimation of the UE's location and movement, assuming that idle UEs have the same mobility behavior as the active ones. Other approaches for estimating the behavior of idle UEs include network simulation [114] and examining traffic density on roads across neighboring cells [29]. Although the technical terms, cell load and handover, generally represent the active UEs, in this dissertation they are considered to represent the distribution and mobility of idle UEs.

\subsection{Location Management}

The Tracking Area (TA) is defined as an area in which a UE may move freely without updating the MME. Therefore, a TA is a logical area-partition of the network, and each partition is a subset of cells having the same Tracking Area Code (TAC) [1]. When an idle UE passes a TA boundary, it sends an uplink signaling message to the MME. This procedure is called tracking area update (TAU). On the other hand, when the network needs to place a call to a UE, the MME sends downlink paging signaling messages to the cells inside the UE's current TA, in order to find the cell from which the UE can receive the call.

There is a great list of literature on location management in cellular networks (readers are referred to $[16,66,96,116]$ for some overview). All the problems related to the planning and optimization of Location Area (LA) in GSM networks and Routing Area (RA) in GPRS and UMTS networks can be generalized to the study of Tracking Area. Throughout this section, the term LA is mostly used, because it is the term found in the related references. However, to avoid confusion, the term UE is kept here. There are some proposed strategies for location management in the literature. In [16], [39], and [116], most of these strategies have been reviewed and categorized. This section summarizes the most frequently studied schemes. These can be categorized into two main sections: location area update schemes, and paging schemes. 


\subsubsection{Update Schemes}

The MME has to hold the most recent TAC for each UE. In order to ensure this, all UEs are required to perform an update when they realize that their current surveying extended Node B (eNB) has a different TAC. The update procedure begins with an update message from the UE over the Random Access Channel (RACH), and that is followed by some signaling which updates the database of the core network. Due to the use of network bandwidth and core network communication, for the purpose of modification of location databases, each update is a costly exercise.

There are several different schemes for reducing the number of update messages from the UEs. Generally, the update schemes are partitioned into two categories: static and dynamic. In the static schemes, the updates are based on the changes in the topology of the network, while in the dynamic ones the updates are based on the user's call and mobility patterns. Static schemes allow efficient implementation and low computational requirements as they are independent of user characteristics. Unlike the static schemes, the dynamic ones usually require the online collection and processing of data, which consumes significant computing power. On the other hand, the dynamic schemes reduce the signaling overheads more than the static schemes. Thus, for dynamic schemes, a careful design is necessary for the network to support the computation effectively [16].

\subsubsection{Examples of Static Update Schemes}

- Always Update: In this scheme, the UE updates its location whenever it moves into a new cell. The network has complete knowledge of the user's location and no paging is required. This scheme performs well for users with low mobility rates and high call arrival rates. However, in practice, this scheme is never used, due to its need for excessive updates.

- Never Update: In this scheme, the UE never updates, which means that the location update overhead is zero. However it leads to excessive paging for large-scale networks as well as for networks with high call arrival rates. This scheme is almost never used either.

- Reporting Cells: In this scheme, the UE updates its location only when visiting one of the predefined reporting cells. To page a UE, a search must be conducted around the vicinity of the last reporting cell from 


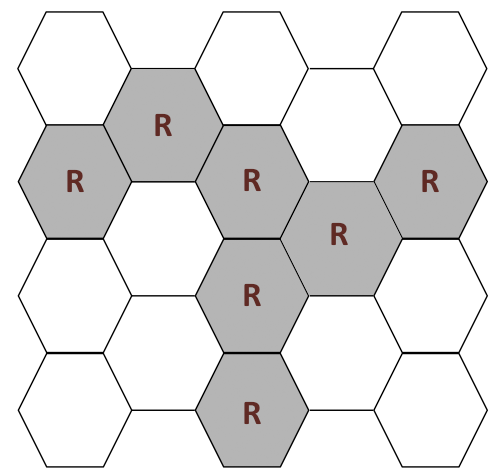

Figure 2.1: The reporting cell scheme with bounded topology.

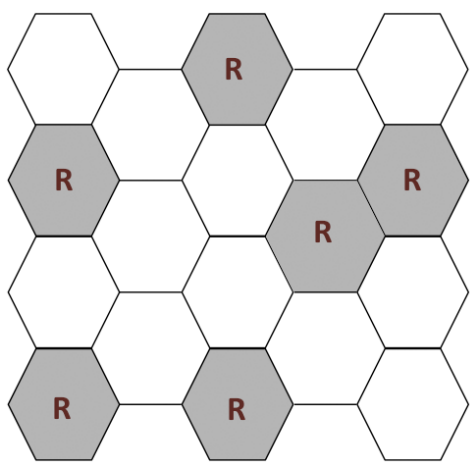

Figure 2.2: The reporting cell scheme with unbounded topology.

which the UE has updated its location [18, 22, 23]. It is not possible to assign an optimum arrangement for the reporting cells without considering the movements of users. It has been proved in [22] that even with knowledge from the network, finding the optimum arrangements of the reporting cells, is an $N P$-hard problem.

The reporting cells scheme has two types of topologies, bounded and unbounded, which are shown in Figures 2.1 and 2.2. In these figures, the hexagonal shapes represent cells, and the dark ones indicate reporting cells. The advantage of the unbounded topology is the fewer number of reporting cells, which results in a reduction of the number of redundant updates. However, this topology requires a more intelligent paging scheme to track the UEs in the unbounded search space. 


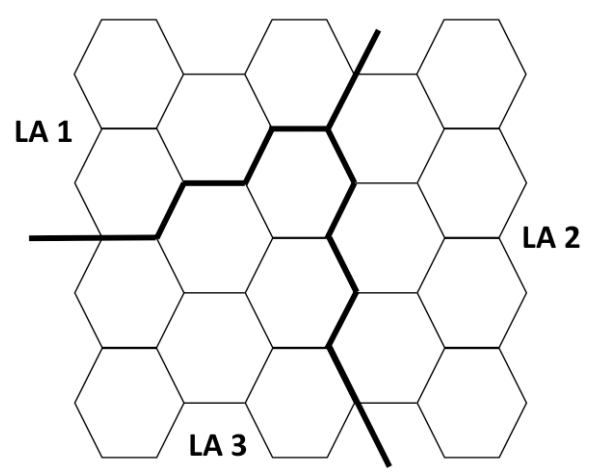

Figure 2.3: LA partitioning.

- Forming LA: In this scheme, the UE performs a location area update (LAU) whenever it changes an LA. The paging of a UE will occur inside the LA in which the UE is located. This scheme is referred to as the standard update scheme, and it is shown in Figure 2.3. The update part of the standard TA scheme which will be explained later and used throughout the dissertation is similar to this scheme.

\subsubsection{Examples of Dynamic Update Schemes}

- Selective LA update: In this scheme, the LAU is not performed every time the user crosses an LA border. The LAU process at certain LAs can be skipped, as the user might spend a very short period of time in those LAs [101].

- Time-based: In this scheme, the UE updates its location at constant time intervals. In Figure 2.4, while moving from point A to B, the UE performs an update every $\Delta t$ time interval. In order to minimize the number of update messages, the time interval can be optimized per user [89].

- Profile-based: In this scheme, the network maintains a profile for each user. The profile has a sequential list of the LAs where the user is most likely to be located at different time periods. The LAs on the list are paged sequentially from the most to the least likely LA where a user can be found. The profile of each user should be updated from time to time $[95,107]$. 


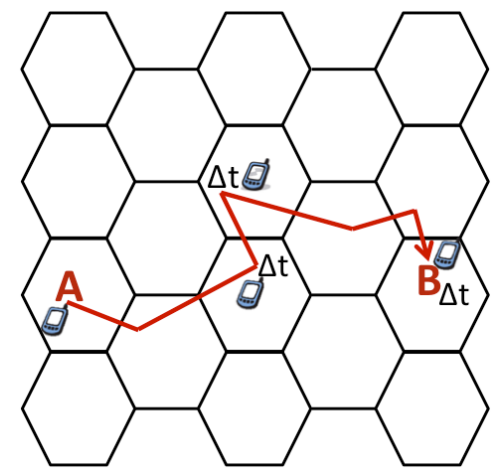

Figure 2.4: The time-based scheme.

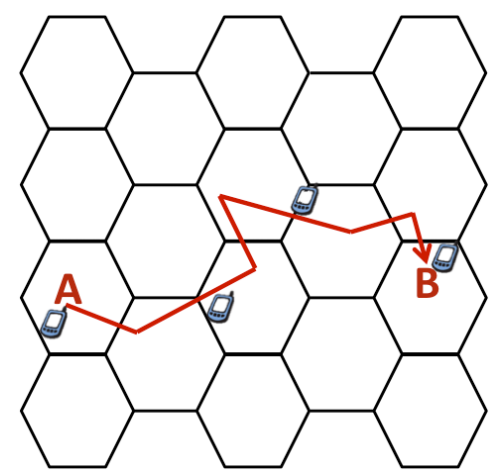

Figure 2.5: The movement-based scheme.

- Movement-based: In this scheme, the UE updates its location after a predefined number of boundary crossings to other cells in the network. In Figure 2.5, when moving from point A to B, the UE performs an update while passing two cell boundaries. The boundary-crossing threshold can be optimized per UE on the basis of its individual movement and call arrival pattern [15].

- Distance-based: In this scheme, the UE updates its location when it has moved a certain distance away from the cell where it last updated its location. Figure 2.6 shows how the UE performs an update when it is one neighbor-hop away from the previous updated cell when moving from point A to B. The distance threshold can be optimized per UE based on its individual movement and call arrival pattern [117]. 


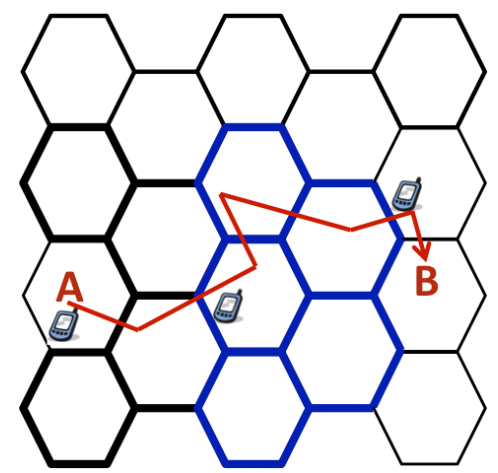

Figure 2.6: The distance-based scheme.

- Predictive distance-based: In this scheme, the network determines the probability density function of the user's location based on location and speed reports. The UE performs an update whenever its distance exceeds the threshold measured from the predicted location [69].

\subsubsection{Paging Schemes}

By paging, the network determines the location of a specific UE to cell level. The MME is the core node responsible for paging. Each step in the attempt of determining the location of a UE is referred to as a polling cycle. When the MME receives a downlink data notification message from the Serving Gateway (SGW), it sends polling signals over the Physical Downlink Control Channel (PDCCH) to all cells where a UE is likely to be present. The Downlink Control Information (DCI), which is transmitted over PDCCH, contains the scheduling assignment for the paging message including the exact identity of the UE being paged. When the UE detects the scheduling assignment as it monitors the PDCCH, it demodulates and decodes the paging message. If the paging message does not contain its identity, the UE discards it, otherwise it sends a service request to the MME.

The paging overhead, which is the result of radio bandwidth usage, is proportional to the number of polling cycles, as well as to the number of cells being polled in each cycle. In each polling cycle there is a timeout period, and if the user is not found in that time frame, another group of cells will be chosen in the next polling cycle. The maximum paging delay depends on the maximum number of polling cycles allowed for finding the 


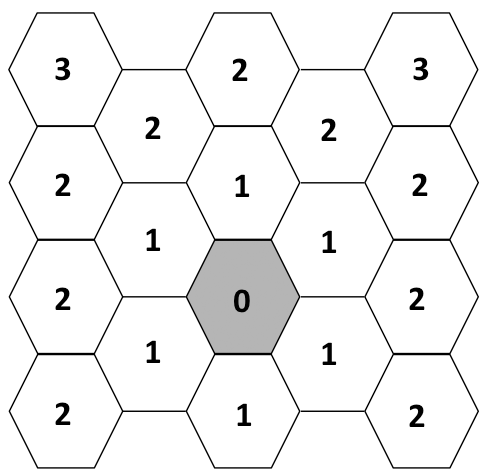

Figure 2.7: The shortest-distance-first scheme.

UE. Because the goal is to reduce the paging overhead, all paging schemes are based on a prediction of where the UE can be located.

\subsubsection{Examples of Paging Schemes}

- Blanket polling (simultaneous paging): In this scheme, all cells in the user's LA are paged simultaneously. This scheme requires no extra knowledge of user location, and it is one of the most practical and commonly used schemes in current networks. In this dissertation, it is also called the standard paging scheme.

- Shortest-distance-first: In this scheme, the network pages the UE by starting from the last cell where the UE has updated its location and moving outward based on the shortest-distance-first order. Figure 2.7 illustrates the sequential paging sets based on the distance from the last updated cell. The numbers in the figure indicate the paging sequence of the group to which each cell belongs.

- Sequential paging: In this scheme, the UE is paged sequentially in sub-groups of cells in the LA. The sub-groups are ordered according to their estimated probabilities of having the UE located in them.

- Velocity paging: In this scheme, the UEs are classified by their velocities at the moments of location updates. In this case, the paging area is dynamically generated on the basis of the user's last LAU time and velocity class index [111]. 


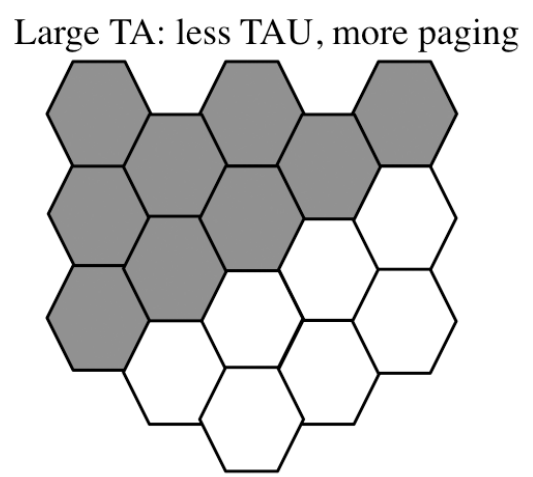

(a)

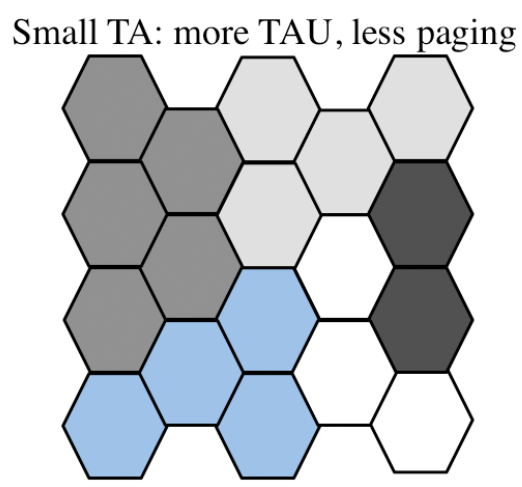

(b)

Figure 2.8: An illustration of the TAU and paging trade-off.

In addition to the above examples, various sequential paging schemes have been proposed in $[15,73,76,95,99,112]$. Although the selective LAU and paging schemes discussed here and in the previous section reduce the signaling overhead, their use requires a modification of system implementation and the collection of additional user information. Hence, the standard scheme is still widely used.

\subsection{Optimization Problems}

Under the standard scheme of TAU and paging, the main design task is the formation of TAs, with the objective of minimizing the total amount of signaling overhead. Having TAs of a very small size (e.g., one cell per TA) virtually eliminates paging, but causes excessive TAU, whereas TAs of too large a size produce the opposite effect. This basic trade-off in TAP is illustrated in Figure 2.8.

A UE trace is defined as the cell-to-cell movement behavior and the call arrival pattern of a UE in a specific time period. Having information related to the UE traces would significantly help to reduce the signaling overhead and optimize the TA configuration [121]. The example below reaches a conclusion that even a rough estimation of the UE traces can be useful in planning and optimizing TAs.

- Example: In Figure 2.9, the UE traces are known for the specified area. In the figure to the left, the UE-traces range shows that there are many 

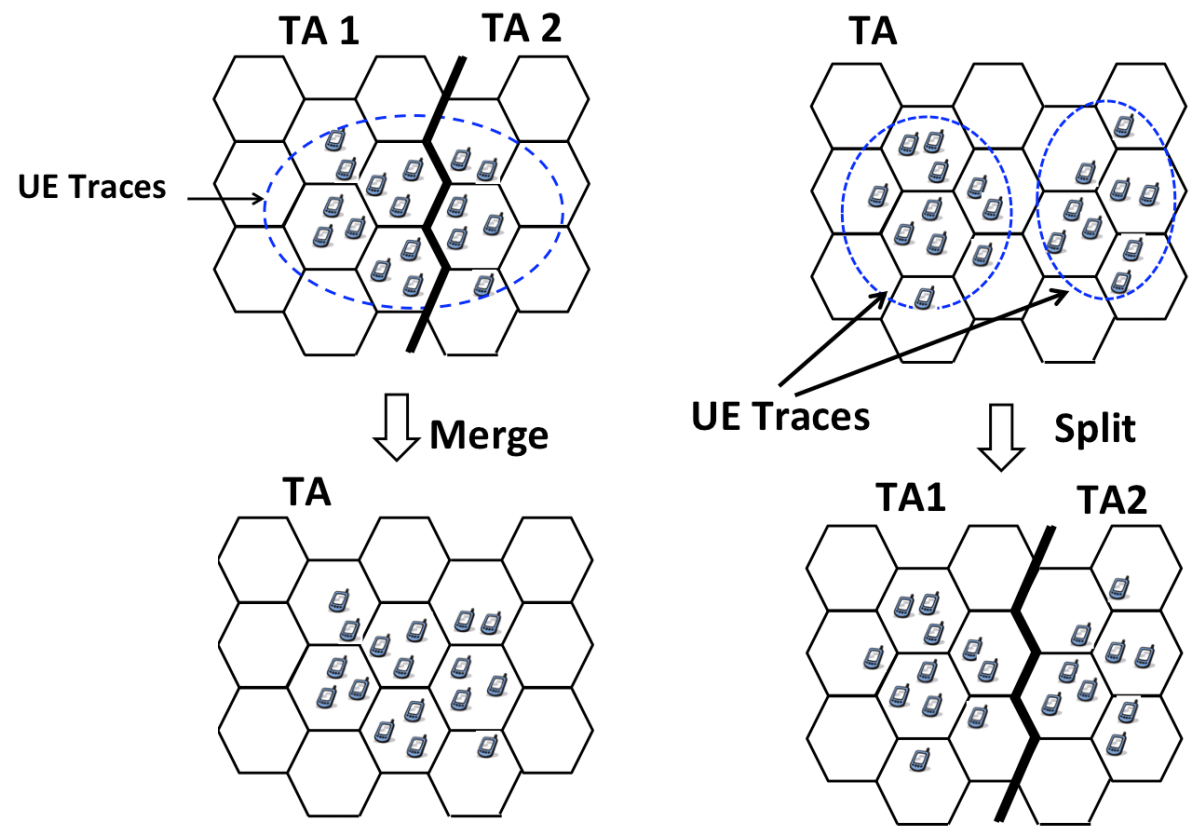

Figure 2.9: Merging and splitting TAs.

UEs crossing the TA border and performing TAU, hence merging the two TAs reduces the number of updates. In the figure to the right, the separation of UE traces indicates that by splitting the TA into two smaller TAs, there are no additional TAUs, while there is less paging. Hence the total signaling overhead is reduced.

From the discussion above, it can be concluded that the natural objective in TAP is to reach an optimal balance between TAU and paging signaling. Tcha et al. [109] applied mathematical programming to a similar problem for GSM. They presented an integer programming model and a cutting plane algorithm, and reported optimality of a GSM network of 38 cells. Because the problem is $N P$-hard, solutions to large networks are typically obtained by heuristic algorithms, such as insertion and exchange local search [94], simulated annealing [42], and genetic algorithms [55]. A heuristic based on the notion of matrix decomposition is presented in [17].

In [100], a host of heuristic algorithms for LA design are evaluated in terms of optimality and computational effort. In addition to LA design, the authors of [100] address cell-to-switch assignment for load balancing. Joint 
LA design and cell-to-switch assignment under the assumption of hexagonshaped cells, is solved by a greedy algorithm in [28]. A simulated annealing algorithm for a similar problem is presented in [43].

Multi-layer LA design, where each LA may contain several paging areas, is solved by simulated annealing in [92]. The authors of [68] provide an integer programming model for this problem and a solution approach based on a graph-partitioning heuristic. In [114], the author makes use of the simulation tools developed by the EU MOMENTUM project [87], originally intended for cell planning, to predict LAU and paging requests. An integer programming model is used for jointly designing LAs, RAs, and UTRAN registration areas (URAs) in [114].

As previously discussed, this dissertation follows the standard TAU and paging scheme for location management. This means that the movement of a UE crossing the TA boundary leads to a TAU message, and paging is performed simultaneously in all cells of the TA to which the UE is currently registered.

\subsection{System Model and Basic Notation}

An eNB (extended Node B, eNodeB) in LTE networks is the equivalent of a base station in GSM networks, and it is the building block of the Radio Access Networks (RANs). Every eNB can serve multiple sectors [n sectors, each of $360 / \mathrm{n}$ degrees]. Each sector is called a cell, and the eNB is commonly referred as the "site". In TA configuration, splitting the cells of a site into different TAs is not a common practice. Therefore, although all the models and theories presented in the dissertation are at cell level, most of the performance evaluations are considered at site level. This does not impose any loss of generality as all the design frameworks generalize straightforwardly to both elements.

Based on the discussion above, the set of cells/sites in a network is denoted by $\mathscr{N}=\{1, \ldots, N\}$, and the set of TAs currently in use is denoted by $\mathscr{T}=\{1, \ldots, T\}$. The vector $\mathbf{t}=\left[t_{1}, \ldots, t_{N}\right]$ is used as a general notation of cellto-TA assignment, where $t_{i}$ is the TA of cell $i$. TA design $\mathbf{t}$ can be alternatively represented by an $N \times N$ symmetric and binary matrix $\mathbf{S}(\mathbf{t})$; in which element $s_{i j}(\mathbf{t})$ represents whether or not two cells are in the same TA, i.e.,

$$
s_{i j}(\mathbf{t})= \begin{cases}1 & \text { if } t_{i}=t_{j} \\ 0 & \text { otherwise }\end{cases}
$$


Two types of input data, representing the UE location and mobility behavior for a given time period of interest, are used for the performance evaluation of a TA design. Let $u_{i}$ be the total number of UEs in cell $i$ (load of $i$ ) scaled by the time proportion that each UE spends in cell $i$. For the same time period, $h_{i j}$ is the number of UEs moving from cell $i$ to cell $j$. The values of $u_{i}$ and $h_{i j}$ can be assessed by the cell load and handover statistics of active UEs. It is very reasonable to assume that the mobility behavior of idle UEs is close or identical to that of active UEs, and hence the cell load and handover statistics can be used for performance evaluation of the signaling overhead.

\subsection{Signaling Overhead Calculation and Unit}

The total update and paging signaling overhead is defined by $c_{S O}(\mathbf{t})$ and is calculated by Equation (2.2). The cost of one paging and one update are denoted by $c^{p}$ and $c^{u}$, respectively. Moreover, parameter $\alpha$ is the call intensity factor/activity factor (i.e., probability that a UE has to be paged).

$$
c_{S O}(\mathbf{t})=\sum_{i \in \mathcal{N}} \sum_{j \in \mathscr{N}: j \neq i}\left(c^{u} h_{i j}\left(1-s_{i j}(\mathbf{t})\right)+\alpha c^{p} u_{i} s_{i j}(\mathbf{t})\right)
$$

Within the outer parentheses of (2.2), the first term accounts for the TAU overhead for UEs moving from $i$ to $j$ (if the two cells are not in the same TA). The second term is the paging overhead introduced in cell $j$ while doing the paging of UEs in cell $i$ (if the two cells are in the same TA).

The exact relationship between $c^{u}$ and $c^{p}$ depends on the radio resource consumption [49], and computing these costs in terms of bytes or money units opens up another line of research. The signaling overhead values in this dissertation have no real physical unit. Hence, these values have no meaning, unless they are compared to another signaling overhead calculated by the same formula.

Apart from the performance evaluation presented in Chapter 3, in the remainder of this dissertation, the cost of a single TAU is set at ten times as much as the cost of a single paging. This ratio is common in the literature $[30,49,66]$. That is if $c^{u}$ is set to 1 cost unit, then $c^{p}$ should be 0.1 cost units. In all the performance evaluations discussed in this dissertation, the call intensity factor is $\alpha=0.05$, assuming that $5 \%$ of the UEs are paged. 


\subsection{Description of the Lisbon Network}

In this section, the description of the Lisbon network is presented, because it is used for performance evaluation throughout this dissertation. To evaluate the performance of the proposed models and algorithms, they are applied to a realistic set of data representing a mobile cellular network for the downtown area of Lisbon. This set of data is provided by the EU MOMENTUM project [87].

The network consists of 60 sites and 164 cells. A reference scenario of the UE distribution and mobility is defined by accumulating the cell load and handover statistics in the data set. Figure 2.10 illustrates the network and the reference scenario. The sites are represented by disks. For every site, its cells are illustrated by squares. The location of a square in relation to its site center shows the direction of cell antenna. The darkness of each cell is in proportion to its accumulated cell load. A link is drawn between two cells if there is any handover between them, and the amount of handover is proportional to the thickness of the link. 


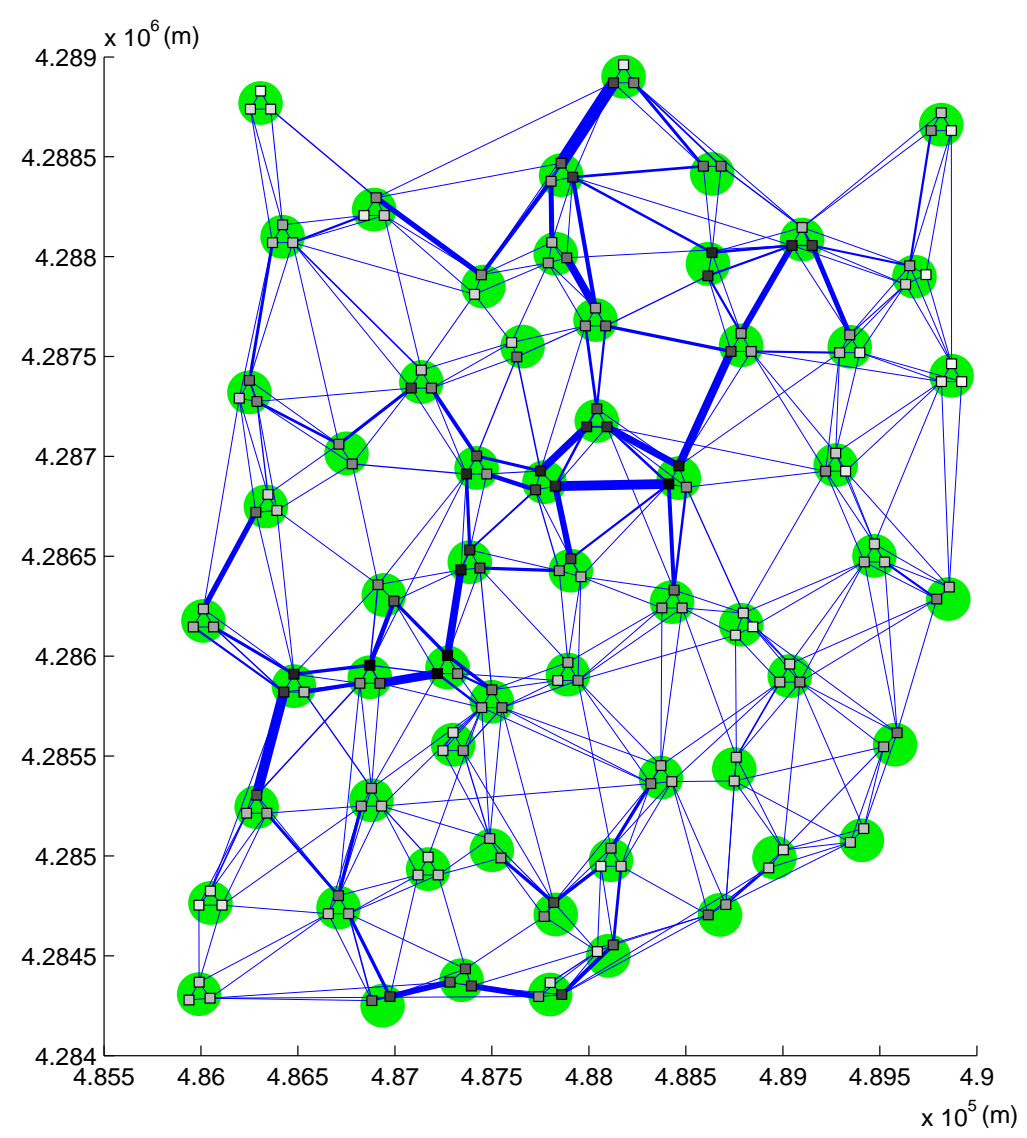

Figure 2.10: An illustration of the Lisbon network, and the reference scenario. 



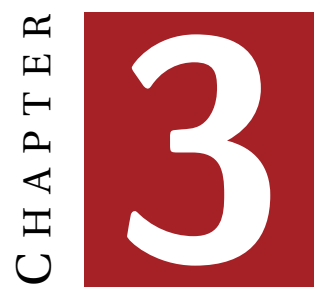

\section{Tracking AREa RE-OPTIMIZATiON}

The earlier optimized TA configuration in the planning phase will not perform satisfyingly after some time, due to changes in UE distribution and mobility patterns. In order to re-optimize the configuration over time, it is not practically feasible to deploy a green-field design, as the configuration might significantly differ from the original one. By re-optimization, the design is successively improved by re-assigning some cells to TAs other than their current ones.

There are two reasons for applying a re-optimization approach. First, reconfiguring TAs, which is typically done by moving a cell from one TA to another, requires temporarily turning off the cell and thus service interruption - a very costly process from the service standpoint. Second, the benefit of a new, optimized TA design gradually diminishes over time as UE location and mobility patterns change. Thus, one has to weigh the performance improvement of some limited time duration against the cost in terms of service interruption due to reconfiguration. The service interruption aspect is calculated by bounding the number of UEs that are affected by TA reconfiguration. Here, this bound is referred as the budget.

In this chapter, a re-optimization approach for revising TA design is presented. The service interruption caused by TA reconfiguration is explicitly taken into account. The complexity and solution characterization of the resulting optimization problem are both investigated. Finally, an algorithm which is able to deliver high-quality solutions in a short computing time is presented. The study in this chapter has previously been published in [79]. 


\subsection{Problem Definition}

The most basic and convenient reconfiguration option is used as the building element of re-optimization; namely to move a cell from its current TA to a new one. That is, the output of the re-optimization process consists of a subset of cells that have the changed TAs and the new TA of each of these cells. Before discussing the details, it is worth pointing out that the gain resulting from the re-optimization, in terms of reduced total paging and TAU overhead, results from the joint effect of the re-assignments, i.e., whether or not a cell should change TA and to which TA the cell should move, both depend on the decisions made for other cells.

For TA re-optimization, the TA design currently deployed in the network is given. This solution is denoted by $\mathbf{t}^{0}$. If the result of re-optimization is $\mathbf{t}^{*}$, then reconfiguration means moving all cells $i$ from $t_{i}^{0}$ to $t_{i}^{*}$ for which $t_{i}^{0} \neq t_{i}^{*}$. The reduction of the number of TAs is allowed, which means that if a TA becomes empty after cell moves, it is simply deleted. To simplify the presentation, increasing the total number of TAs is not considered, although the algorithm can be easily extended to include this option.

For every cell, a parameter is defined to represent the cost in service interruption if the TA of the cell is changed. For convenience and without loss of generality, the UE distribution parameter $u_{i}$ is used to measure the amount of service interruption of cell $i$. Let $\mathbf{d}\left(\mathbf{t}, \mathbf{t}^{0}\right)$ be a binary vector representing cells that have been assigned new TAs, that is, $d_{i}\left(\mathbf{t}, \mathbf{t}^{0}\right)=1$ if and only if $t_{i}^{0} \neq t_{i}, i \in \mathscr{N}$. Denoting the budget value by $B$, the following budget constraint is introduced.

$$
\sum_{i \in \mathscr{N}} u_{i} d_{i}\left(\mathbf{t}, \mathbf{t}^{0}\right) \leq B
$$

The TA re-optimization (TAR) problem is formalized below.

[TAR] Find a TA design $\mathbf{t}$ that satisfies the budget constraint (3.1) and minimizes the total overhead cost $c_{S O}(\mathbf{t})$ as defined in Section 2.2.

A closely related problem, which is considered in most of the references in Chapter 2, is to make a TA design completely from scratch. Here, this green-field-design problem is referred as tracking area optimization (TAO). The optimum to TAO is a lower bound to the best achievable performance of TAR. This value will be used as a reference in performance evaluation. 


\subsection{Complexity and Solution Characterization}

TAR turns into TAO if the budget constraint is removed. TAO is known to be $N P$-hard [109]. Bejerano et al. [24] showed that TAO remains NP-hard even over a star (i.e., one cell is the only and common neighbor to all other cells).

The above facts do not prove that TAR is NP-hard. The TAR complexity is formalized in the following proposition if we assume that (3.1) is nonredundant.

PROPOSITION 1. TAR remains NP-hard when the budget constraint (3.1) is non-redundant.

PROOF. The TAR problem can be restated as (3.2), which minimizes the total signaling overhead subject to the budget constraint.

$$
\begin{array}{ll}
\min & c_{S O}(\mathbf{t}) \\
\text { s.t. } & \sum_{i \in \mathscr{N}} u_{i} d_{i}\left(\mathbf{t}, \mathbf{t}^{0}\right) \leq B
\end{array}
$$

Observing that (3.2b) resembles the binary knapsack constraint [75], it can be shown that any instance of the knapsack problem given in (3.3) can be transformed to an instance of (3.2).

$$
\begin{aligned}
\max & \sum_{i=1}^{n} v_{i} x_{i} \\
\text { s.t. } & \sum_{i=1}^{n} w_{i} x_{i} \leq W \\
& x_{i} \in\{0,1\}
\end{aligned}
$$

Given any instance with $n \geq 1, v_{i} \geq 0, w_{i} \geq 0$ for $i=1, \ldots, n$ and $W \geq 0$ of knapsack problem, an instance of TAR is defined as follows:

$$
\begin{aligned}
& \mathscr{N}:=\{1, \ldots, 2 n\}, \alpha=1, c^{u}=1, c^{p}=0, u_{i}:=\left\{\begin{array}{ll}
w_{i} & \text { if } i=1, \ldots, n \\
w_{i-n} & \text { if } i=n+1, \ldots, 2 n
\end{array},\right. \\
& h_{i j}:=\left\{\begin{array}{ll}
v_{i} & \text { if } j=i^{\prime}, i, j \in \mathscr{N} \\
0 & \text { otherwise }
\end{array}, B:=W .\right.
\end{aligned}
$$

There are $2 n$ cells forming $n$ pairs of cells $\mathscr{N}:=\left\{1,1^{\prime}, \ldots, i, i^{\prime}, \ldots, n, n^{\prime}\right\}$. The initial solution $\mathbf{t}^{0}$ is defined as every cell forms its own TA. In the transformation, every item in the knapsack problem corresponds to moving either cell $i$ to TA of $i^{\prime}$, or cell $i^{\prime}$ to TA of $i$, with an equivalent effect. Doing 
either of the two moves lead to an improvement in the total signaling overhead. The improvement is equal to the objective function coefficient of the knapsack instance. Moreover, no additional improvement is possible other than these moves. Making such a move consumes $u_{i}$ units of $B$. Finally, merging the two cells of any cell pair is independent of that of other pairs. Then any feasible solution of the knapsack problem corresponds to a feasible solution of the defined TAR with the same objective instance values, and vice versa, and the theorem follows.

The following proposition provides a solution characterization.

PROPOSITION 2. If there is no budget limit and any number of TAs is allowed, then a solution is non-optimal if it contains any TA of which the cells can be partitioned into two (or more) subsets $\mathscr{N}_{1}$ and $\mathscr{N}_{2}$, such that there is no handover between the subsets, i.e., $h_{i j}=0$ for all $i \in \mathscr{N}_{1}$ and $j \in \mathscr{N}_{2}$.

PROOF. Suppose the cells in $\mathscr{N}_{1}$ form a new TA. The TA update overhead does not increase, because any update due to user mobility from any cell in $\mathscr{N}_{1}$ to another TA exists before the new TA is formed, and there are no user movements between the cells in $\mathscr{N}_{1}$ and those in $\mathscr{N}_{2}$. The paging overhead goes down due to TA split. Hence the conclusion.

What is stated in Proposition 2 is, in fact, very intuitive from a network planning point of view: Assuming that the amount of handover $h_{i j}>0$, if and only if cell $i$ and $j$ are geographically adjacent, then in an optimal design of TAO, every TA consists of geographically connected cells. For TAR, the result does not always hold in theory because of the budget constraint and the limitation of using at most $T$ TAs. Nevertheless, it tends to be satisfied for practically relevant planning scenarios. This greatly reduces the computational effort in the repeated local search algorithm which will be presented in Section 3.3.

Although the resulting complexity of TAR makes use of the knapsack problem, the former is considerably harder in practice, simply because the changes in the total signaling overhead due to cell moves are dependent on each other.

- Example: Figure 3.1 illustrates the dependency between cell moves by using a simple example of two TAs and seven cells. The boundary between the TAs is shown by the thick lines. All cells have $u$ UEs, and 


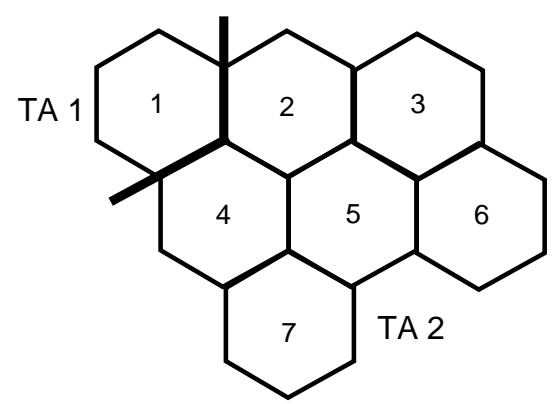

(a) Moving any single cell leads to higher overhead

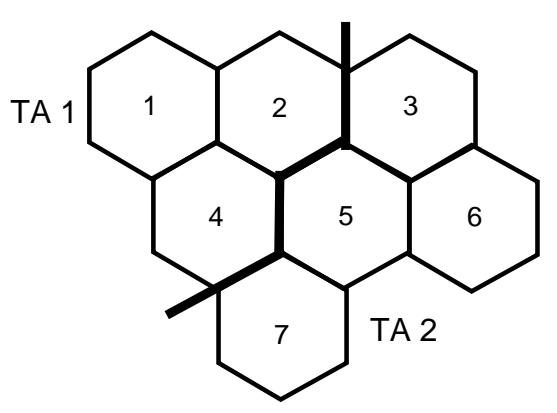

(b) Improvement by moving two cells

Figure 3.1: An example of the dependency between cell moves.

the amount of handover in both directions together is $h$ for all pairs of adjacent cells. For simplicity, let $c^{u}=c^{p}=1$, and $\alpha=0.1$. The total signaling overhead of the current TA design is $2 h+3 u$ (Figure 3.1(a)). Assume $h$ is between $0.4 u$ and $0.6 u$. It can be verified that moving any single cell from its TA to the other TA (including moving cell 1 and making TA 1 empty) results in higher total overhead. However, there is an improvement if both cells 2 and 4 are moved to TA 1 (Figure 3.1(b)).

The above example illustrates the phenomenon of local optima. Problem TAR is further complicated by the budget constraint, because a collection of cell moves may not be feasible. The solution algorithm presented in Section 3.3 considers these aspects by allowing for some non-improving moves, but limiting the amount of budget they may consume.

\subsection{A Solution Approach Based on Repeated Local Search}

Solving TAR to optimality may require excessive computational effort in view of its complexity. In this chapter, a simple but effective heuristic algorithm is developed using repeated local search to find high-quality solutions rapidly. 


\subsubsection{Local Search}

The local search algorithm iteratively updates the TA design. In every iteration, the algorithm considers cells that may be moved in respect of the remaining budget, and among these cells selects the cell move that results in the largest improvement. This is repeated until no additional move of any cell is allowed because of the budget limitation or until no further improvement can be obtained.

In its first repetition, the initial solution is $\mathbf{t}^{0}$, and the local search behaves like a greedy algorithm that successively builds up a solution of TAR. In subsequent runs, solution initialization follows the procedure in Section 3.3.2. The local search algorithm is formalized in Algorithm 1, in which the solution given to and returned by the algorithm is denoted by $\mathbf{t}^{\ell}$.

Because $\mathbf{t}^{\ell}$ is not necessarily equal to $\mathbf{t}^{0}$ when the algorithm starts, some cells may have been moved from their original TAs in the initial solution $\mathbf{t}^{\ell}$. Therefore, in Step 4, which constructs the set of cells to be considered for move, the budget constraint (3.1) is checked only if a cell is still in its original TA, as otherwise the corresponding contribution to the left-hand side of (3.1) is already accounted in $b^{\ell}$. For the same reason, in Step 19, $b^{\ell}$ decreases (i.e., some of the budget becomes released) if a cell is moved back to its original TA.

In Step 6, the set $\mathscr{T}^{\prime}$ contains candidate TAs to which cell $i$ may be moved. Motivated by Proposition 2, TAs that at present do not have any cell with a positive handover value to cell $i$ are excluded. As a result, the size of $\mathscr{T}^{\prime}$ is much smaller than $T-1$, leading to a significant speed-up of the algorithm. In theory, excluding TAs in this way may overlook some possible improvements, whereas in practice, there is no noticeable performance degradation.

\subsubsection{Repeated Local Search}

Additional improvements can be obtained by applying the local search algorithm repeatedly using different starting solutions. However, to be effective, the initial solutions should satisfy two conditions. First, there must be some slack budget to allow for moving cells from their original TAs. Second, the initial solution should not be a completely randomized one (with a very high total signaling overhead), as otherwise no good solution can be found before the entire budget is consumed. Moreover, from the structure of TAR, it is expected that good solutions will have some cell moves in common. 


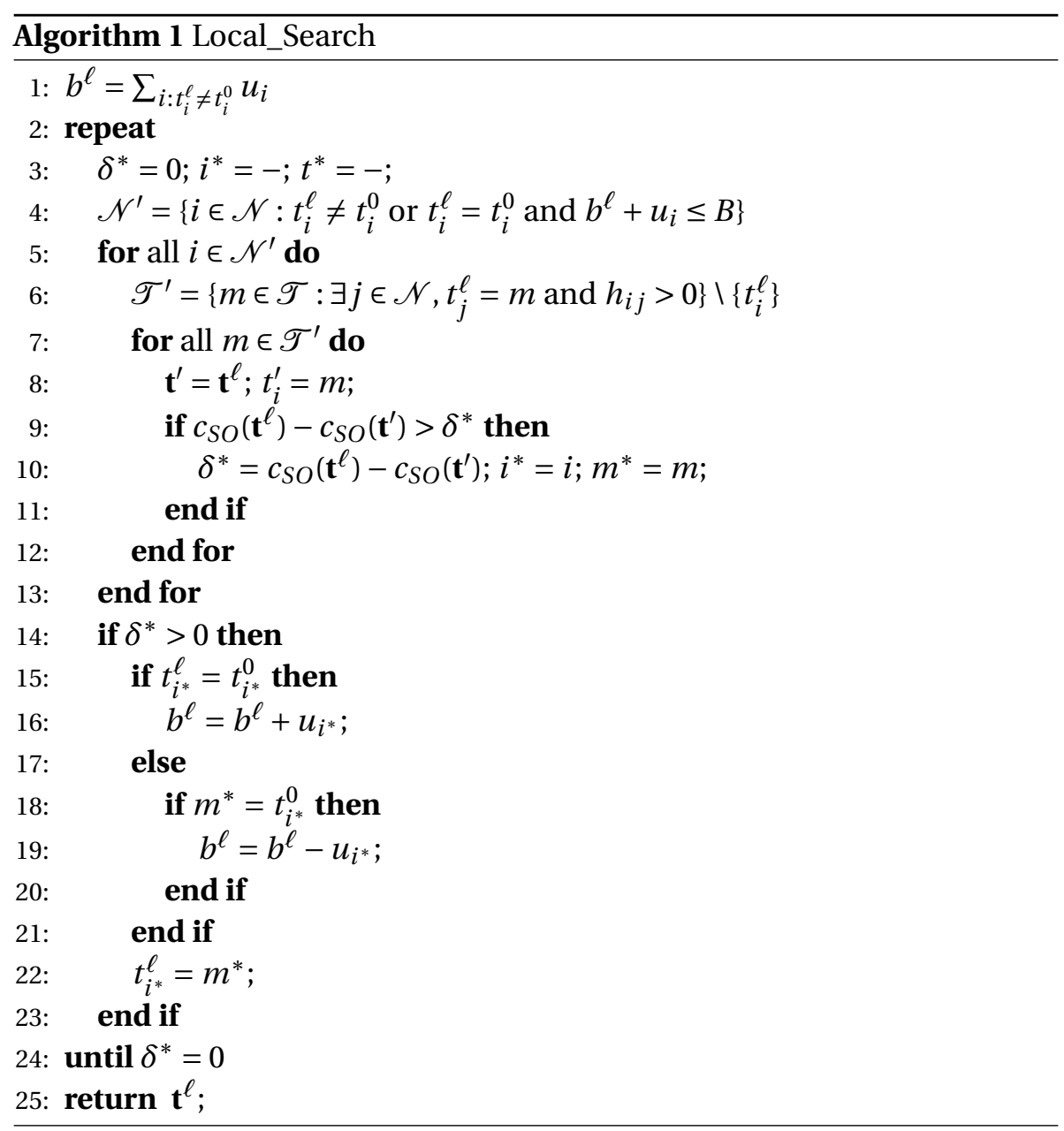

Based on the above observations, an initial solution is constructed as follows. Let $\mathbf{t}^{*}$ be the best solution so far. Cells are partitioned into two subsets $\mathscr{N}^{0}$ and $\mathscr{N}^{1}$, containing cells that remain in the same TA as in the original design $\mathbf{t}^{0}$, and cells that have been assigned to new TAs by $\mathbf{t}^{*}$, respectively. A two-step perturbation to $\mathbf{t}^{*}$ is applied. Two budget parameters, $B^{1}$ and $B^{0}$, with $B^{1}<B^{0}<B$, are used. In the first step of the perturbation, some randomly chosen cells in $\mathscr{N}^{1}$ are moved back to their original TAs in $\mathbf{t}^{0}$, such that the consumed budget becomes less than or equal to $B^{1}$, that is, the amount of slack is at least $B-B^{1}$. Next, some cells in $\mathscr{N}^{0}$, again chosen randomly, are moved from their TAs to new ones, until the consumed budget reaches $B^{0}$. Moving a cell $i \in \mathscr{N}^{0}$ to a new TA is performed in a greedy 
manner. That is, the cell is moved to the TA giving the largest improvement, if such a TA exists, otherwise the cell is moved to the TA that results in the minimal increase in overhead. This second step of perturbation is aimed at exploring improvements that come from the joint effect of multiple cells presented in Section 3.2, although none of these moves alone results in improvement.

Algorithm 2 formalizes the repeated local search algorithm. In the first step, local search is applied to the original TA design $\mathbf{t}^{0}$. Then perturbation, combined with local search, is performed $K$ times.

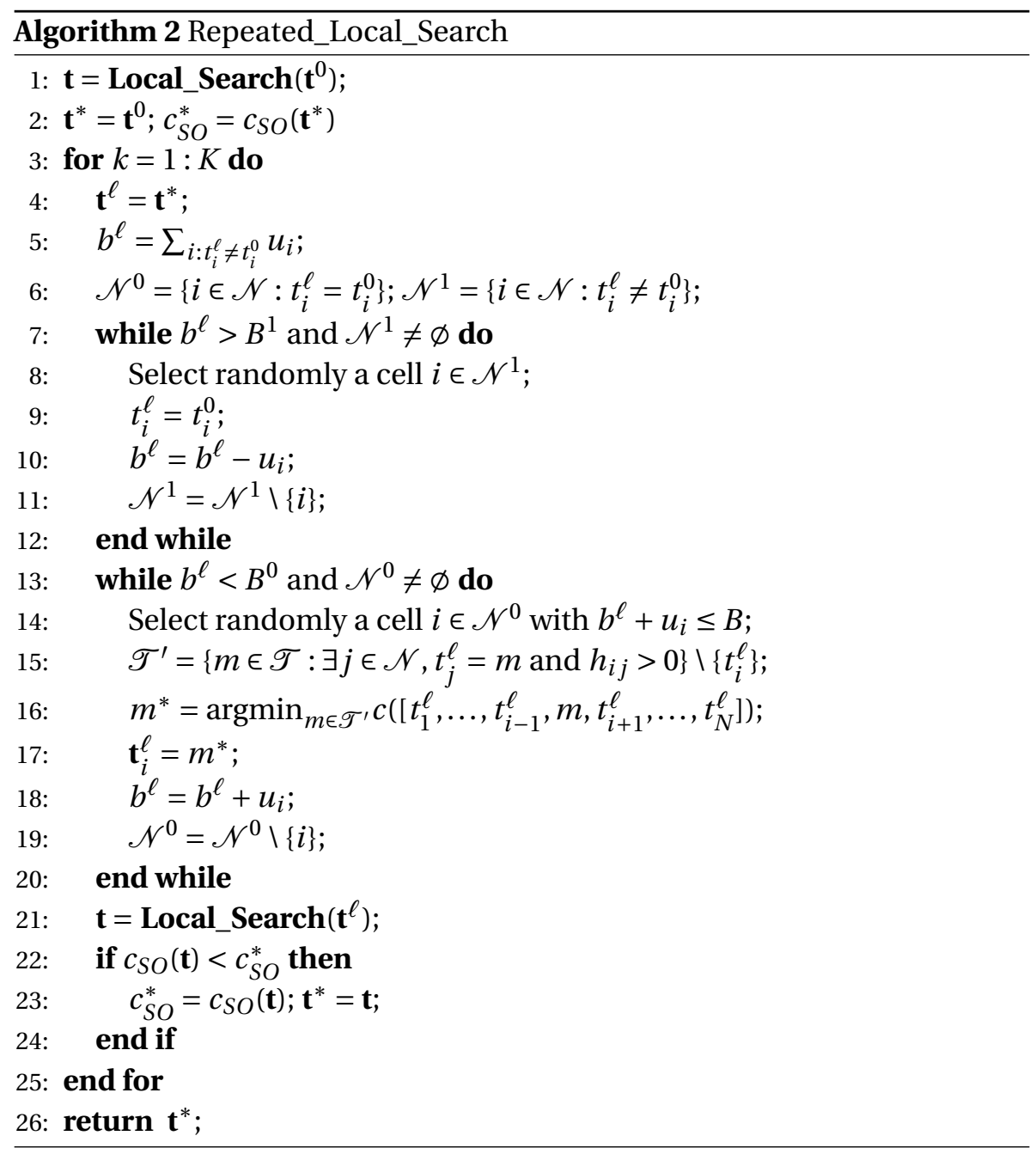




\subsection{Numerical Results}

Here, the results of performance evaluation using realistic data from the Lisbon network are presented. Besides the reference scenario shown in Figure 2.10, two additional scenarios (I and II) were generated by modifying the cell load and handover statistics. Scenario II has a larger deviation from the reference compared to scenario I. Provided that the location and mobility patterns have evolved from the reference scenario into each of the two scenarios, the TA re-optimization is conducted. Figure 3.2 illustrates scenario I in the same format as that used for the reference scenario. In all three scenarios, $5 \%$ of the UEs are paged in every cell (i.e., $\alpha=0.05$ ). The overhead of a single TAU $c^{u}$ is set at twice as much as $c^{p}$.

The reference scenario in Figure 2.10 represents UE location and mobility patterns to which $\mathbf{t}^{0}$ is optimal. For this optimization, the model in [109] and software CPLEX [60] have been used. Computing the solution is timeconsuming. In practicing TAR, $\mathbf{t}^{0}$ is the design currently in use, and hence this computation is not needed.

The resulting TA design $\mathbf{t}^{0}$ is shown in Figure 3.3. There are 44 TAs in the design. In the figure, two cells are connected by an edge if and only if they are in the same TA. Thus, TAs are represented by fully connected subsets of cells. One can observe that if two cells have a large amount of handover in Figure 2.10, then they are in the same TA in Figure 3.3.

In addition to $\mathbf{t}^{0}$, the optimal green-field TA designs for scenarios I and II are also computed and denoted by $\mathbf{t}^{*}$ (I) and $\mathbf{t}^{*}$ (II), respectively. The two solutions are attainable only if re-optimizing TAs disregarding the budget constraint is allowed. Like computing $\mathbf{t}^{0}$, finding these two solutions is hardly feasible for large-scale networks. For the Lisbon network they can be obtained, although the computing time is long. In order to assess the effectiveness of the algorithm, $\mathbf{t}^{*}$ (I) and $\mathbf{t}^{*}$ (II) are used as bounds on the best achievable performance of TAR.

In the repeated local search algorithm, $B^{1}=0.85 B, B^{0}=0.95 B$, and $K=$ 100 are set. The computing time is about 30 seconds on a notebook with Intel Core 2 Duo processor at $2.0 \mathrm{GHz}$. For each of the scenarios I and II, two budget levels of $B$, corresponding to $5 \%$ and $15 \%$ of the total cell load, i.e., $B=B^{\prime} \cdot \sum_{i \in \mathcal{N}} u_{i}$ where $B^{\prime}=5 \%$ and $15 \%$, are used. For performance evaluation, the algorithm without budget limitation $\left(B^{\prime}=100 \%\right)$ is also run and compared to $\mathbf{t}^{*}$ (I) and $\mathbf{t}^{*}$ (II).

Table 3.1 gives the numerical assumptions and values for this performance evaluation and the computational results are summarized in Table 


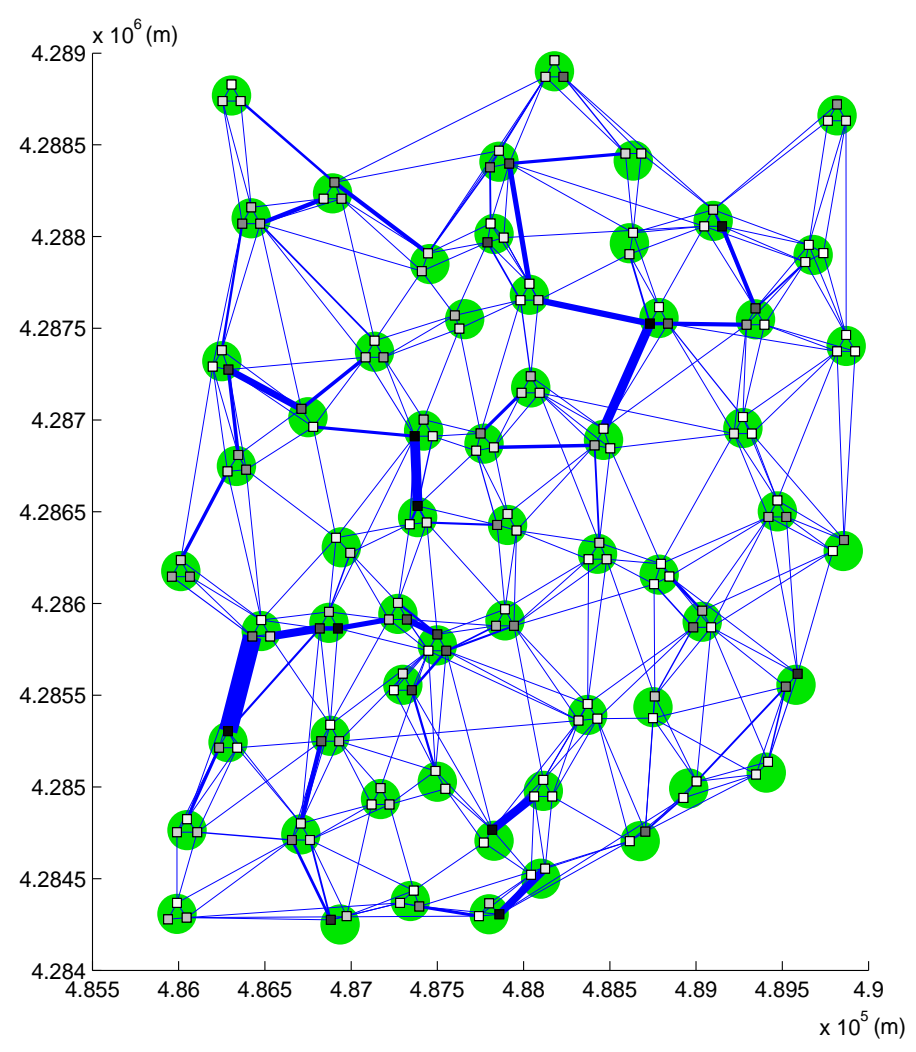

Figure 3.2: An illustration of scenario I.

3.2. For the two scenarios, the total signaling overhead values of the initial TA design are shown in row $\mathbf{t}^{0}$. These values represent the TA performance when the initial TA design $\mathbf{t}^{0}$ is kept for the two scenarios. The results of how much re-optimization improves TA performance for the two budget levels are also reported $\left(B^{\prime}=5 \%\right.$ and $\left.B^{\prime}=15 \%\right)$. The last row shows the optimal solutions with unlimited budget and number of TAs.

From the table, it can be observed that the original TA design $\mathbf{t}^{0}$, optimized for the reference scenario, is about $20 \%$ and $36 \%$ away from optimum for scenarios I and II, respectively. By running the local search once, it was possible to improve $\mathbf{t}^{0}$ considerably. An additional amount of improvement was obtained by repeated local search. The improvement grew when $B^{\prime}$ in- 


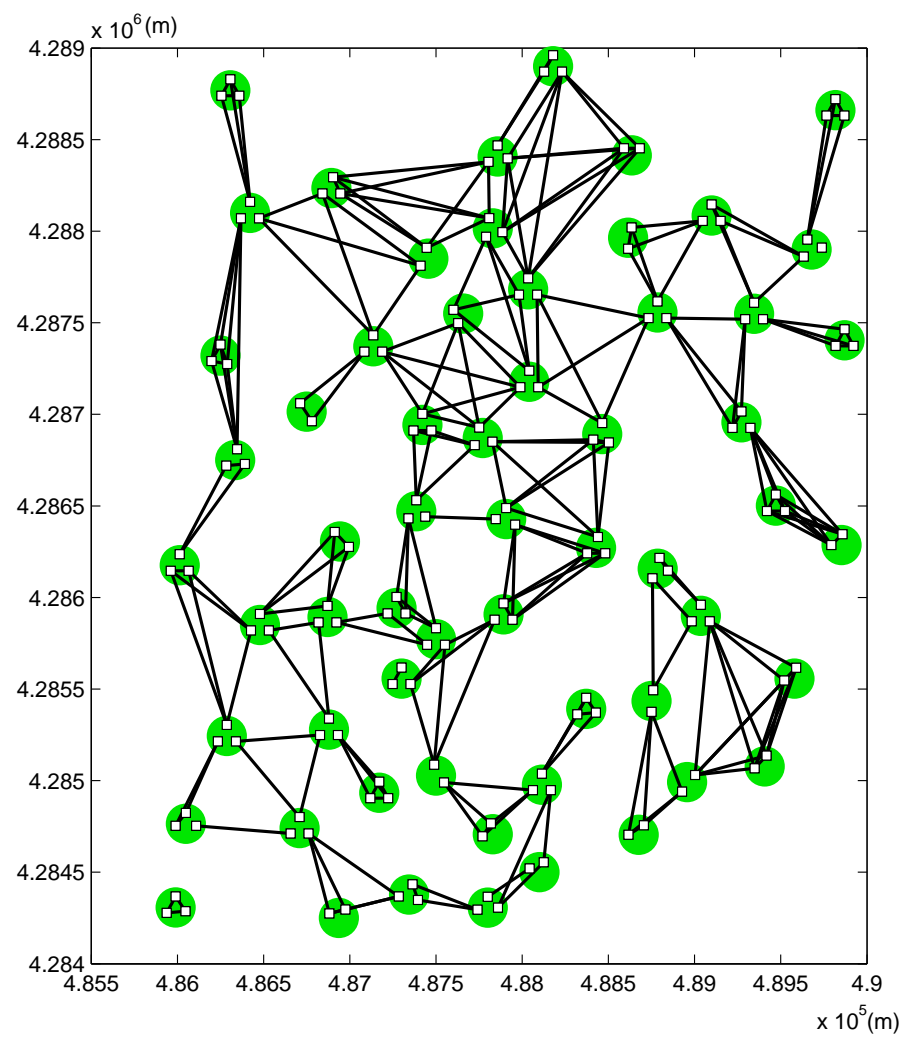

Figure 3.3: TA design $\mathbf{t}^{0}$ (optimum of the reference scenario).

Table 3.1: Numerical assumptions and values for performance evaluation.

\begin{tabular}{lll}
\hline Parameter & Description & Values \\
$\alpha$ & Call internsity factor & 0.05 \\
$c^{p}$ & Cost of a single paging & 0.5 \\
$c^{u}$ & Cost of a single TAU & 1 \\
$B^{\prime}$ & Total cell load factor corresponding to the budget level & {$[0.05,1]$} \\
$K$ & Number of iteration & 100 \\
$N$ & Number of cells & 164 \\
$T$ & Number of TAs in $\mathbf{t}^{0}$ & 44 \\
\hline
\end{tabular}

creased from $5 \%$ to $15 \%$; the difference is larger for scenario II because its 
UE distribution and mobility patterns deviate more from the reference scenario. Moreover, for both scenarios, there is no difference in the solutions of local search for $B^{\prime}=15 \%$ and $B^{\prime}=100 \%$. In other words, local search is not able to further improve its solution even when more budget was made available, because the algorithm already reaches a local optimum for $B^{\prime}=15 \%$. The results of repeated local search show its strength of overcoming this issue. The effectiveness of repeated local search is further demonstrated by the solutions for $B^{\prime}=100 \%$. In this case, the algorithm's performance is very close to the optimal - the deviation is less than $1 \%$ for both scenarios.

Table 3.2: Results of TA re-optimization.

\begin{tabular}{lcccc} 
(LS = Local search; RLS = Repeated local search. $)$ \\
\hline \multicolumn{4}{c}{$\mathbf{t}^{0}$} & \multicolumn{2}{c}{ Scenario I } & \multicolumn{2}{c}{ Scenario II } \\
& 292.68 & \multicolumn{2}{c}{386.62} \\
$B^{\prime}=5 \%$ & 261.52 & 257.13 & 386.62 & 380.03 \\
$B^{\prime}=15 \%$ & 257.56 & 250.25 & 376.42 & 354.96 \\
$B^{\prime}=100 \%$ & 257.56 & 245.70 & 376.42 & 336.96 \\
& \multicolumn{2}{c}{$\mathbf{t}^{*}(\mathrm{I})=243.05$} & \multicolumn{2}{c}{$\mathbf{t}^{*}(\mathrm{II})=333.73$} \\
\hline
\end{tabular}

Figure 3.4 illustrates the re-optimized TA design for scenario I and $B^{\prime}=$ $5 \%$. In total, 21 cells have changed TAs. These cells are marked in color (red) in the figure. Comparing the solution to $\mathbf{t}^{0}$, one can see that re-optimization adapts TA design from the reference scenario given in Figure 2.10 to scenario I, which was presented in Figure 3.2. For example, the cell pointed out by the horizontal arrow in Figure 3.4 changed TA. This change is most likely due to the fact that the growth in its UE mobility has been transferred to another cell. At one site, indicated by the vertical arrow, the three cells that were in the same TA have been split into different TAs as a result of fewer UEs in these cells.

\subsection{Conclusions}

A re-optimization approach has been presented to adapt a given TA design to trends and changes in UE location and mobility patterns. The approach is novel as it accounts the cost of reconfiguring TAs by means of a budget constraint. This is justified by the fact that once a TA design is in use, adopting a new solution of green-field optimization is typically not feasible or does 


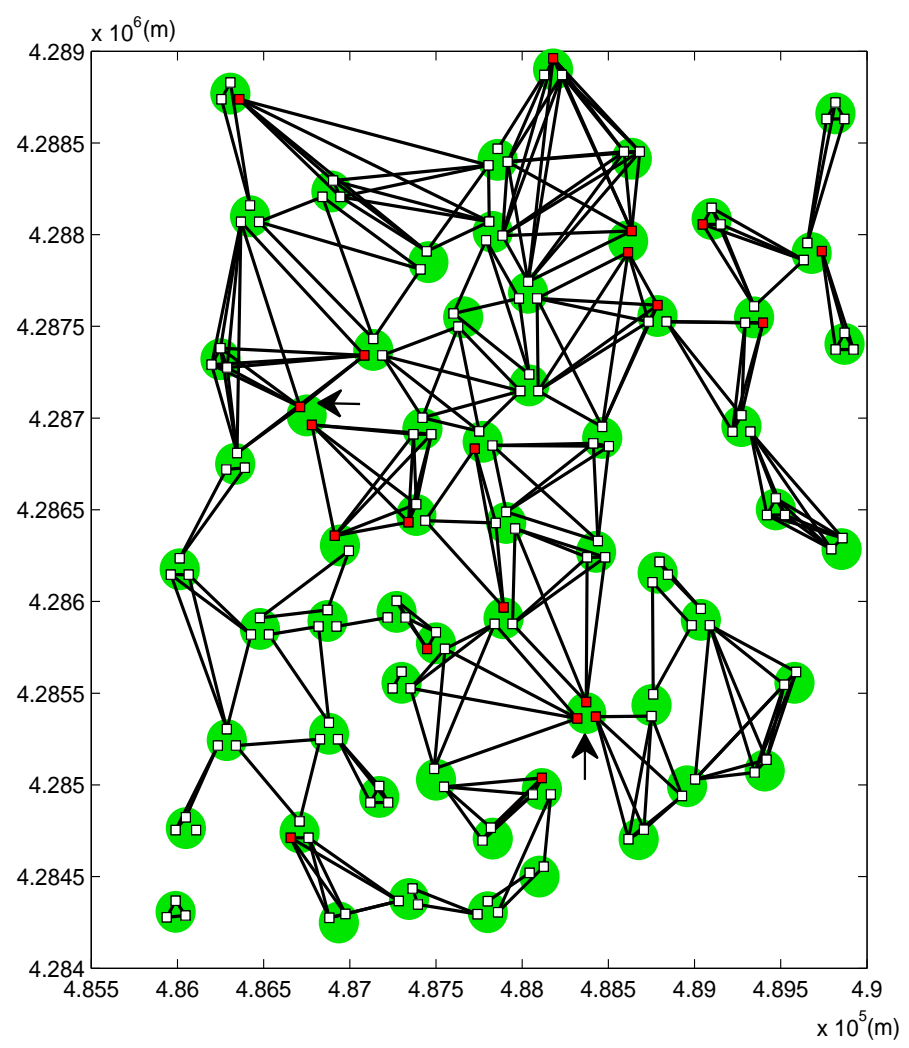

Figure 3.4: Re-optimized TA design for scenario I, $B^{\prime}=5 \%$.

not pay off in real networks. The complexity of the problem was investigated and a fast algorithm based on repeated local search was developed. The case study on a realistic network showed that the algorithm is able to approach high-quality solutions. 



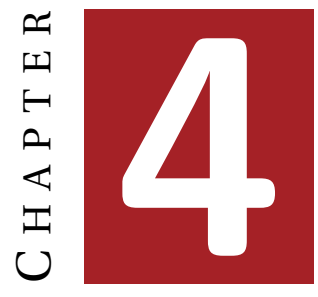

\section{TRADE-OFF IN TA RECONFIGURATION}

Reconfiguring TA usually requires restarting the cells which are changing TAs, and consequently results in service interruption. For a network operator, it is therefore important to realize the trade-off between approaching minimum signaling overhead and the cost resulting from the reconfiguration. In this chapter, a bi-objective optimization framework is proposed to solve this trade-off.

Unlike mono-objective optimization problems which have unique optimal values, in bi-objective problems the solution set is formed by Paretooptimal (non-dominated) points. An integer programming (IP) model is developed to optimize the overhead by reconfiguration, given a specific cost budget constraint. Applying the proposed model to various budget levels leads to a set of Pareto-optimal solutions. The IP model may have to be run many times, depending on the number of Pareto-optimal solutions and the fact that for some budget levels, the model may find an already obtained Pareto-optimal solution. Therefore, solving the IP model is time-consuming and sometimes infeasible for large-scale networks.

To deal with large-scale networks, a genetic algorithm (GA) embedded with local search (LS) is proposed. The algorithm searches for Pareto-optimal solutions in one single run. Contrary to the approaches that use a scalarization function or treat the various objectives separately, the GA approach uses the concept of dominance in the fitness evaluation. In the GA algorithm, the amount of dominance explicitly evaluates each solution in terms of Pareto optimality.

The performance of the proposed integer model and GA algorithm is demonstrated through experiments using three large-scale realistic/real-life network scenarios. For the first two scenarios, it was possible to compare 
the results from the GA algorithm with the ones computed from the IP model. The last network was only solved by the GA algorithm, since it was too large it was not feasible to solve it with the IP model. The results demonstrate the ability of the approaches to deliver various Pareto-optimal solutions, thus giving the operator the opportunity of selecting a proper trade-off between the two objectives. The research presented in this chapter has appeared in $[80,85]$.

\subsection{System Model}

The system model considered in this chapter is an extension of the definitions described in Sections 2.4 and 3.1, with some modifications described below. The signaling overhead, $c_{S O}(\mathbf{t})$, follows (2.2). The cost of reconfiguration is denoted by $c_{R}(\mathbf{t})$, and it is computed by (4.1), where $t^{0}$ is the TA design currently deployed in the network. Equation (4.1) follows the cost definition in the previous chapter.

$$
c_{R}(\mathbf{t})=\sum_{i \in \mathscr{N}} u_{i} d_{i}\left(\mathbf{t}, \mathbf{t}^{0}\right)
$$

The aim is to observe the trade-off between $c_{S O}(\mathbf{t})$ and $c_{R}(\mathbf{t})$ of the design $\mathbf{t}$; thus, the problem is modeled with the following bi-objective formulation.

$$
\begin{gathered}
\min \left(c_{S O}(\mathbf{t}), c_{R}(\mathbf{t})\right) \\
\text { s.t. } s_{i j}(\mathbf{t})= \begin{cases}1 & \text { if } t_{i}=t_{j} \\
0 & \text { otherwise }\end{cases} \\
d_{i}\left(\mathbf{t}, \mathbf{t}^{0}\right)= \begin{cases}1 & \text { if } t_{i}^{0} \neq t_{i}, \\
0 & \text { otherwise }\end{cases}
\end{gathered}
$$

\subsection{An Integer Programming Model}

To solve the bi-objective problem formulated in (4.2a)-(4.2c), one approach is to minimize $c_{S O}(\mathbf{t})$ defined in (2.2) for various reconfiguration cost budgets. In other words, the TA re-optimization problem is solved repeatedly for different limits on $c_{R}(\mathbf{t})$. By denoting the budget value by $B$, the budget corresponds to the constraint $c_{R}(\mathbf{t}) \leq B$ in the binary IP model. The model has two sets of binary variables: 
- $s_{i j}(\mathbf{t})$ is 1 when $i$ and $j$ are in the same TA and 0 otherwise.

- $p_{i t}$ is 1 when cell $i$ belongs to TA $t$ and 0 otherwise.

$$
\begin{array}{ll}
\min & \sum_{i \in \mathscr{N}} \sum_{j \in \mathscr{N}: j \neq i}\left(c^{u} h_{i j}\left(1-s_{i j}(\mathbf{t})\right)+\alpha c^{p} u_{i} s_{i j}(\mathbf{t})\right) \\
\text { s. t. } & \sum_{t \in \mathscr{T}} p_{i t}=1, \\
& p_{i t}+p_{j t}-1 \leq s_{i j}(\mathbf{t}), \\
& s_{i j}(\mathbf{t})+p_{i t}-1 \leq p_{j t}, \\
& s_{i j}(\mathbf{t})+s_{j k}(\mathbf{t})-s_{i k}(\mathbf{t}) \leq 1, \\
& \sum_{i \in \mathscr{N}} u_{i}\left(1-p_{i t_{i}^{0}}\right) \leq B .
\end{array}
$$$$
\forall i, j, k \in \mathscr{N}, i \neq j \neq k
$$

In the model presented, constraint (4.3b) assures that each cell is assigned to only one TA. Constraints (4.3c) and (4.3d) define the matrix $\mathbf{S}(\mathbf{t})$ and the correlation between $s_{i j}(\mathbf{t})$ and $p_{i t}$. When $p_{i t}=p_{j t}=1$, it means that $i$ and $j$ are in the same TA $t$, and hence $s_{i j}=1$ as imposed by constraint (4.3c). If $p_{i t}=1$ and $p_{j t}=0$, then $i$ belongs to TA $t$ while $j$ does not, and therefore $s_{i j}(\mathbf{t})=0$ (constraint (4.3d)). Constraint (4.3e) ensures the transitive property that if two cells $i$ and $k$ belong to the same TA as cell $j$, they must also be in the same TA. That is, if $s_{i j}(\mathbf{t})=s_{j k}(\mathbf{t})=1$, constraint (4.3e) becomes $s_{i k}(\mathbf{t}) \geq 1$, forcing $s_{i k}(\mathbf{t})=1$. Constraint (4.3f) bounds the number of UEs affected by reconfiguration using the budget level. From the definition of the variable $p_{i t}$, it is clear that $p_{i t_{i}^{0}}$ is one when cell $i$ belongs to the current TA $t_{i}^{0}$ and zero otherwise.

For $B=0$, the current $\mathbf{t}^{0}=\left[t_{1}^{0}, t_{2}^{0} \ldots t_{i}^{0} \ldots t_{N}^{0}\right]$ is the only feasible solution. The signaling overhead of this configuration is unlikely to be optimum, but on the other hand, the corresponding cost is zero. This point is among the Pareto-optimal solutions, as one cannot find any solution with a better reconfiguration cost. Other Pareto-optimal solutions can be calculated by giving other values of $B$.

\subsection{Dominance-based Approach}

Depending on the scale of the network, the solution space of the problem can be very large as it is a combinatorial bi-objective problem. To approach 
high quality solutions, two aspects should be considered. One is the convergence to the Pareto optimal front, and the other when there is diversity in the search procedure. In view of this and the complexity results in Section 3.2, applying meta-heuristics is motivated to deal with this problem for large-scale networks and to deliver the Pareto-optimal solutions in a single run. Multi-objective meta-heuristics can be classified into four main categories based on their solution evaluation strategies.

- Scalar approaches transform the problem into a mono-objective problem. A typical example is the weighted sum method, which combines the objective functions by non-negative weights and converts them into one objective function [61]. Another example would be the goal programming method that uses a target value for each objective function, and the overall goal is to minimize the deviation from the target values [32].

- Criterion-based approaches which treat the various incommensurable objectives separately, such as the parallel approach [93] and the lexicographic approach [48]. In the latter approach, to evaluate a solution against another, the two objective function vectors are compared lexicographically.

- Indicator-based approaches which use performance quality indicators as a search guide [120].

- Dominance-based approaches use the concept of dominance in solution evaluation [13].

Among the approaches above, weighted sum is a frequently used method for solving multi-objective optimization. However, this approach is not used for the problem here for three reasons: First the problem is a combinatorial bi-objective problem as the configuration solutions are described by discrete variables. Thus, the number of Pareto-optimal solutions can be exponential in the problem size [44]. Second, there may exist Pareto-optimal solutions which cannot be the result of any weighted sum of the objective functions. Third, to obtain a diverse set of Pareto-optimal solutions by the weighted sum approach, multiple runs of different weight combinations are required. In general, setting the weights is a difficult task.

To evaluate the solutions, a dominance-based approach is used by defining a parameter called Preference Value (PV) for each solution t. PV(t) is the 


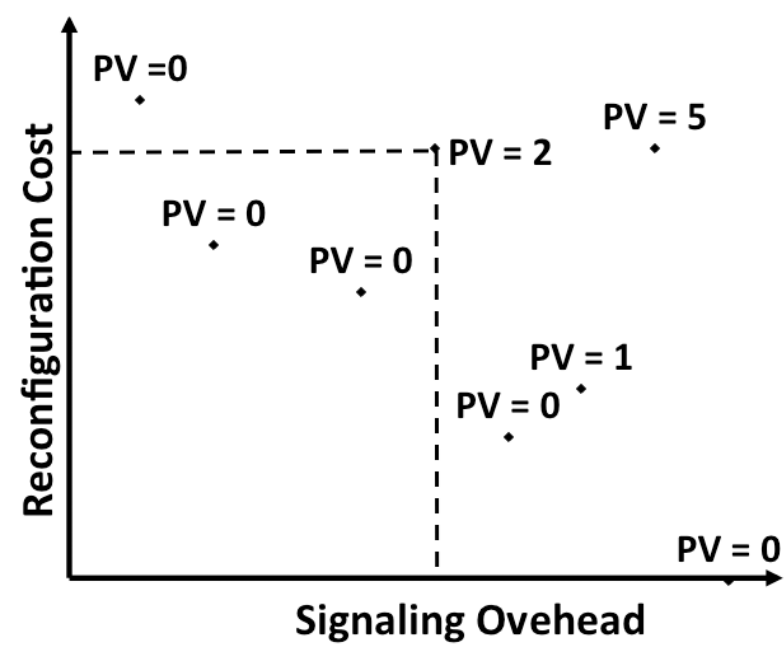

Figure 4.1: An illustration of the PV definition.

number of other solutions which are dominating solution $\mathbf{t}$. This means that a solution with PV $=0$ is among the Pareto-optimal ones.

- Example: Figure 4.1 shows the PV values for a set of solutions. Consider the signaling overhead cost $c_{S O}(\mathbf{t})$ and the reconfiguration cost $c_{R}(\mathbf{t})$ as the two objective functions of the problem. In this figure, there is a point that is dominated by two other solutions, therefore for this point $\mathrm{PV}=2$. The points with $\mathrm{PV}=0$ are the Pareto-optimal solutions.

\subsection{Heuristic Algorithm}

Genetic Algorithm (GA) [54] embedded with Local Search (LS) [12] is used. The two reasons for choosing a GA approach are:

1. The encoding of solutions is simple as it uses integer-valued vectors.

2. The desired Pareto-optimal solutions form a "population" of solutions. Thus, a population-based meta-heuristic approach is a reasonable algorithm candidate.

For solution encoding, a fixed length vector of size $N$ is used. The elements in the vector represent the TA numbers to which the cells belong. Figure 4.2 illustrates the solution vector representation. 


\begin{tabular}{|c|c|c|c|c|c|c|c|c|c|c|}
\hline $\mathbf{1}$ & $\mathbf{2}$ & $\mathbf{3}$ & $\mathbf{4}$ & $\mathbf{5}$ & $\mathbf{6}$ & $\bullet$ & $\bullet$ & $\mathbf{N}-1$ & $\mathbf{N}$ \\
\hline TA & TA & TA & TA & TA & TA & & $\bullet$ & & TA & TA \\
$\mathbf{1}$ & $\mathbf{1}$ & $\mathbf{2}$ & $\mathbf{1}$ & $\mathbf{4}$ & $\mathbf{3}$ & & & & T & T \\
\hline
\end{tabular}

$\mathbf{N}=$ Number of cells

Figure 4.2: Solution vector representation.

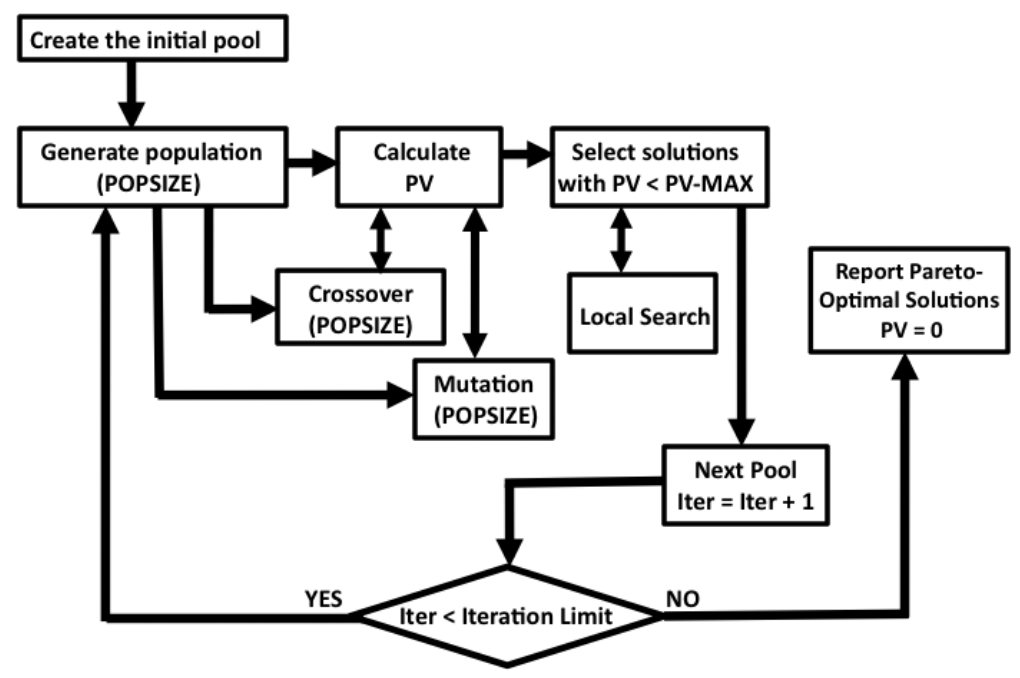

Figure 4.3: Principle design in finding Pareto-optimal configurations.

Figure 4.3 summarizes the principle design of the solution algorithm. In this figure, POPSIZE is the population size considered in GA. The sizes of the outputs from the crossover and mutation operators are also set to POPSIZE. The initial pool is a set of high-quality solutions which are considered in the initial phase of the algorithm. Iteration Limit is used in the termination criterion for the algorithm. The PV threshold, which is used to identify the eligible solutions to be selected for the next population, is denoted by PVMAX. Note that after some iterations, the PV of a Pareto-optimal solution might change and not remain zero. In this case, the solution is taken out of the Pareto optimal set. The output of the algorithm is the solutions with PV $=0$. More explanation of each step will be given in the coming sections. 


\subsubsection{Population Initialization}

Generating the first population of a GA plays an important role in approaching good solutions rapidly. The population must be rich enough to enable high-quality solutions. In order to set the first population, an initial pool is generated.

The current TA configuration $\mathbf{t}^{0}$, which is among the Pareto-optimal solutions, is a natural starting point. To create diversity in the initial pool, the local search algorithm discussed in Section 3.3.1 is applied. Starting from $\mathbf{t}^{0}$, the local search algorithm iteratively updates the TA design. In every iteration, the algorithm considers cells that may be moved, and from among these cells selects the cell move that results in the largest improvement in signaling overhead (cf. the local search algorithm in Chapter 3). This is repeated, without accounting for the reconfiguration cost, until no further improvement can be obtained. In Section 3.3.1 the goal was to find the optimum reconfiguration with a budget limit, while here, the goal is to keep all configurations encountered on the way to the lowest found signaling overhead. The initial pool consists of all the configuration points visited by the local search algorithm. Figure 4.4 illustrates the local search procedure in obtaining the configuration points. The dashed arrows represent the possible moves from $\mathbf{t}^{n}$ to $\mathbf{t}^{n+1}$, where $n$ is the iteration counter. The solid arrows show the moves with largest improvement in the signaling overhead.

From Figure 4.4, it can be observed that while the local search starts from $\mathbf{t}^{0}$ and searches for configuration points with lower signaling overhead, the reconfiguration cost of those points are successively higher. The reason is that more cells change configuration compared to the initial design $\mathbf{t}^{0}$.

All points in the initial pool are considered inside the first population. For generating the rest of the population, the GA algorithm randomly picks a configuration from the initial pool and perturbs the TA configuration of $20 \%$ of the cells. This is repeated until the population size reaches POPSIZE. To avoid poor configurations, in perturbation process, a cell can change TA, only if it is geographically located on the boundary of its TA. This is the case if the cell has at least one neighboring cell with positive handover and the neighboring cell is currently assigned to a different TA. In addition, the new TA is picked from among the TAs of the neighboring cells.

\subsubsection{Crossover}

The role of the crossover operator in GA is to inherit some characteristics of the two parents to generate the offspring [108]. The PV values of the en- 


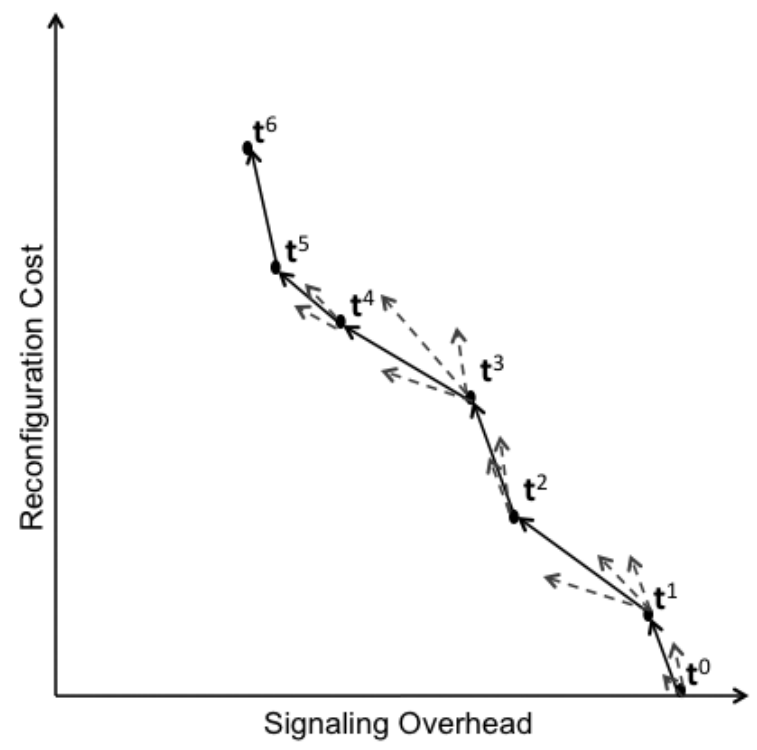

Figure 4.4: Applying local search to create the initial pool.

tire population are calculated. In the crossover operation, two parents are chosen randomly with the preference of having lower PV values. The elements are swapped between the two randomly chosen points to make two offspring. Figure 4.5 explains the 2-point crossover method applied in this study. It is apparent from the figure that the cells in each offspring follow the TA assignment that one of the parents has, and therefore the output offspring from the crossover operator are valid TA design solutions.

In the GA algorithm, the crossover operation is repeated until the number of offspring is equal to POPSIZE. In order to avoid identical offspring, the algorithm first makes sure that the chosen parents are different, and second that the two crossover points are chosen with the condition that the two parents differ in at least one position between the two points.

\subsubsection{Mutation}

The mutation operator randomly modifies the elements of TA configuration vectors to promote diversity. A configuration is randomly chosen from the population with the preference of having low PV to enter the mutation operator. In the selected configuration, $5 \%$ of the elements are mutated. In the GA algorithm, the mutation operation is repeated in POPSIZE times. Similar 


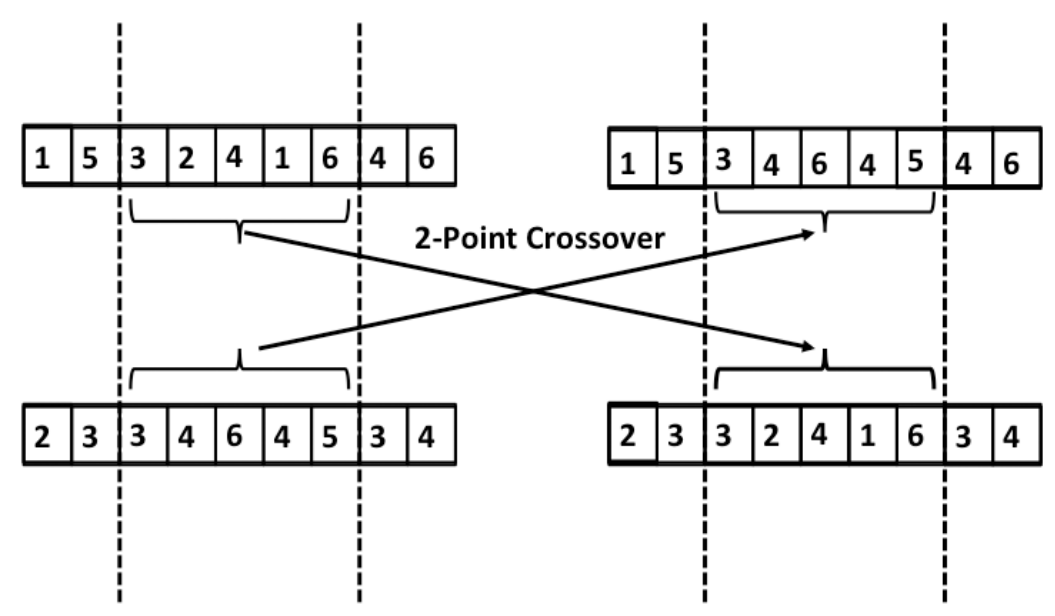

Figure 4.5: The 2-point crossover method in GA.

to the perturbation procedure described in Section 4.4.1, the mutation of a cell may take place only if the cell is on the boundary of its TA, and the TA of that cell can only be changed to a neighboring TA.

\subsubsection{PV Local Search Algorithm}

It is often the case that by some simple modifications, the solutions obtained from the GA can usually be improved. In this study, a PV Local Search (LS) algorithm is used to further strengthen the algorithm during each iteration of the GA. For each solution given to LS, the algorithm considers moving cells to other neighbor TAs one by one. Among these moves, the first move which results in a lower PV value is chosen, as long as the point defined by the signaling overhead and reconfiguration cost has not yet been visited. If the LS gets stuck in a situation where no move results in an unvisited point with a lower PV, the algorithm moves to an unvisited point with an equal PV. The algorithm stops if all possible moves lead to visited points or higher PV values. All points visited by LS are stored and considered as visited in later runs of the LS.

The goal of using LS in this stage of the GA is to first find new solutions with a lower PV to improve the GA performance, and second to look for new Pareto-optimal solutions. The next pool in the GA algorithm consists of solutions with PV $<$ PV-MAX after the LS.

It is possible to tune the number of points entering the LS by giving a 
value to PV-MAX. For example, by setting PV-MAX = POPSIZE, all the points will be considered as an input to LS. PV-MAX is set to be lower than POPSIZE to save computing effort in case of large-scale networks.

\subsection{Efficiency Improvement}

There are two computational bottlenecks in the GA algorithm suggested: First, the PV of a solution is a relative value with respect to other solutions. Therefore, in order to calculate and update the PV of each solution, its signaling overhead and cost should be compared to all other solutions. Second, points that are visited should be stored in order to avoid being generated repeatedly. Ideally, one would like to record all the solution points found by the algorithm in all iterations. However, this becomes computationally unaffordable, since the number of accumulated solutions grows rapidly from one iteration to another. In this section, a method to resolve these bottlenecks is proposed by quantizing the two objective values.

The quantization of the bi-objective value space approximates the signaling overhead and the reconfiguration cost by a fixed and large number of intervals. With this process, the very large set of possible configuration points is approximated by a grid. Figure 4.6 gives an illustration of the quantization. Each pixel of the grid represents all TA configurations which give the signaling overhead and reconfiguration cost within the value intervals defining that pixel. To use this grid in practice over the signaling overhead and cost axes, the grid is mapped to a matrix with the same dimensions as the grid size. Two matrices of same dimension are defined and referred as the visited matrix and the PV matrix. Each of them will help solve one of the two mentioned bottlenecks.

\subsubsection{Visited Matrix}

The visited matrix is defined to keep track on the specific value intervals visited by the algorithm. A binary matrix is used to illustrate whether a grid element has so far been visited or not. If an element of this matrix is one, it means that the corresponding point has been already visited by a solution that has a signaling overhead and cost within that pixel, otherwise the value is zero. The upper part of Figure 4.7 shows a small example of how the visited matrix gets updated while new solutions are found by the algorithm. Moving from the first matrix to the third (left to right), one solution is added in each step to the visited matrix by changing one element from zero to one. 


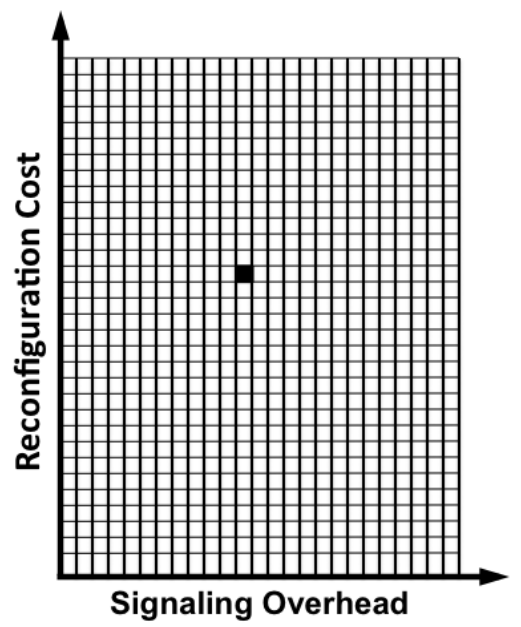

Figure 4.6: Quantization of the overhead and the reconfiguration cost.

(1)

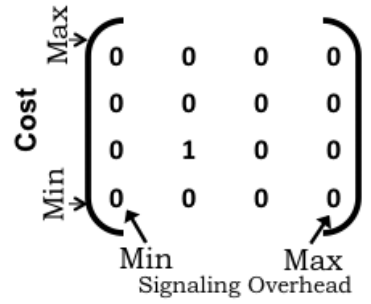

(1)

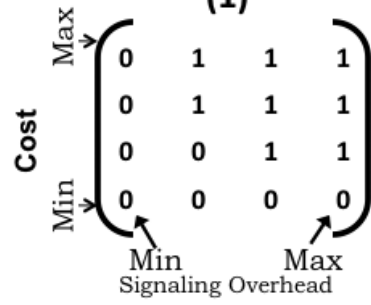

(2)

$$
\left(\begin{array}{llll}
0 & 0 & 0 & 0 \\
0 & 0 & 1 & 0 \\
0 & 1 & 0 & 0 \\
0 & 0 & 0 & 0
\end{array}\right)\left(\begin{array}{cccc}
0 & 0 & 0 & 0 \\
0 & 0 & 1 & 0 \\
0 & 1 & 0 & 0 \\
0 & 0 & 1 & 0
\end{array}\right)
$$
visited matrix

(3)
(2)

$$
\left(\begin{array}{llll}
0 & 1 & 2 & 2 \\
0 & 1 & 1 & 2 \\
0 & 0 & 1 & 1 \\
0 & 0 & 0 & 0
\end{array}\right)\left(\begin{array}{cccc}
0 & 1 & 3 & 3 \\
0 & 1 & 2 & 3 \\
0 & 0 & 2 & 2 \\
0 & 0 & 0 & 1
\end{array}\right)
$$

Figure 4.7: An example of the visited and PV matrices.

\subsubsection{PV Matrix}

In order to find out the PV of a solution in constant time, a PV matrix is defined. This matrix has the same dimension as the visited matrix, and it is 
used to calculate the PV of each solution. Each new solution found by the algorithm is used to update the PV matrix by increasing all the dominated elements to the right and up of the corresponding pixel of the new solution by one. With this method of updating the PV matrix, it can be concluded that at any time, the value of each element in the matrix represents the number of found solutions which dominate the solution of the corresponding element. The lower part of Figure 4.7 illustrates a small example of how to update the PV matrix while adding a new solution. Note that the Pareto-optimal solutions are the elements which are one in the visited matrix and zero in the PV matrix. Thus in the figure, the elements in the boxes represent Paretooptimal solutions.

\subsection{Performance Evaluation}

In this section, results of the performance evaluation for realistic/real-life data of three large-scale networks are presented. In all scenarios, it is assumed that $5 \%$ of the UEs are paged in every site $(\alpha=0.05)$. The overhead of a single TAU $c^{u}$ is set as ten times as much as $c^{p}$ [66]. Table 4.1 gives the numerical assumptions and values for performance evaluation of the three networks.

For each of the first two networks, a reference scenario of UE distribution and mobility is defined. The scenario consists of load and handover statistics of the network. The initial TA configuration, $\mathbf{t}^{0}$, is optimal for the reference scenario. Another UE scenario, called scenario I is generated by modifying the load and the handover statistics. It is considered that the reference scenario has evolved to scenario I over time. The aim is to find the Pareto-optimal solutions of TA reconfiguration for scenario I. The third network is a real-life case, and $\mathbf{t}^{0}$ is the configuration that has been used in the past few years. The suggested algorithm is applied to find the Paretooptimal solutions for reconfiguring $\mathbf{t}^{0}$ for the up-to-date UE distribution and mobility data.

The integer programming model defined in Section 4.2 has been implemented in the Gurobi optimizer [56]. The solver has been run on a processor with the clock speed of $2.4 \mathrm{GHz}$ and $7 \mathrm{~GB}$ available RAM. For the first network, the integer programming model delivers all the exact Pareto-optimal solutions. For the second network, some but not all of the exact Paretooptimal solutions can be calculated by the integer programming model. In this case, due to the size of the third network and to memory limitation, the integer programming model cannot be applied in this case. 
Table 4.1: Numerical assumptions and values for performance evaluation.

\begin{tabular}{lll}
\hline Parameter & Description & Values \\
$\alpha$ & Call intensity factor & 0.05 \\
$c^{p}$ & Cost of a single paging & 0.1 \\
$c^{u}$ & Cost of a single TAU & 1 \\
Iteration Limit $_{1}$ & Limit on the number of iterations for Network 1 & 10 \\
Iteration Limit $_{2}$ & Limit on the number of iterations for Network 2 & 10 \\
Iteration Limit $_{3}$ & Limit on the number of iterations for Network 3 & 3 \\
$N_{1}$ & Number of cells in Network 1 & 164 \\
$N_{2}$ & Number of cells in Network 2 & 225 \\
$N_{3}$ & Number of cells in Network 3 & 978 \\
POPSIZE $_{1}$ & GA population size for Network 1 & 100 \\
POPSIZE & GA population size for Network 2 & 100 \\
POPSIZE 3 & GA population size for Network 3 & 300 \\
PV-MAX $_{T_{1}}$ & PV threshold & 20 \\
$T_{2}$ & Number of TAs in $\mathbf{t}^{0}$ of Network 1 & 7 \\
$T_{3}$ & Number of TAs in $\mathbf{t}^{0}$ of Network 2 & 22 \\
& Number of TAs in $\mathbf{t}^{0}$ of Network 3 & 6 \\
& The PV and visited matrices size for Network 1 & $650 \times 700$ \\
& The PV and visited matrices size for Network 2 & $700 \times 700$ \\
\hline
\end{tabular}

The GA algorithm presented in Section 4.4 is implemented in MATLAB. The computations are run on a processor of type Intel Core 2 Duo with the clock speed of $2.1 \mathrm{GHz}$. For the three networks, the sizes of the visited matrix and PV matrix are chosen to deliver a sufficiently high resolution.

\subsubsection{Network 1}

The first set of data is the Lisbon network studied previously. The optimum configuration for the reference scenario, $\mathbf{t}^{0}$, is computed by the model in [109]. There are seven TAs in $\mathbf{t}^{0}$. Figure 4.8 shows the Pareto-optimal solutions found for the Lisbon network by the two approaches. The black dots represent the exact Pareto-optimal solutions found by the integer programming model. There are 25 Pareto-optimal solutions in Figure 4.8. However, the model has been run more than 25 times in order to find these points. The model takes an average time of about 20 minutes to find a solution for a given cost budget (B). Therefore, for finding these 25 points by the integer programming model, about 8 hours and 20 minutes have been spent. The plus signs in Figure 4.8 illustrate the heuristic Pareto-optimal solutions ob- 


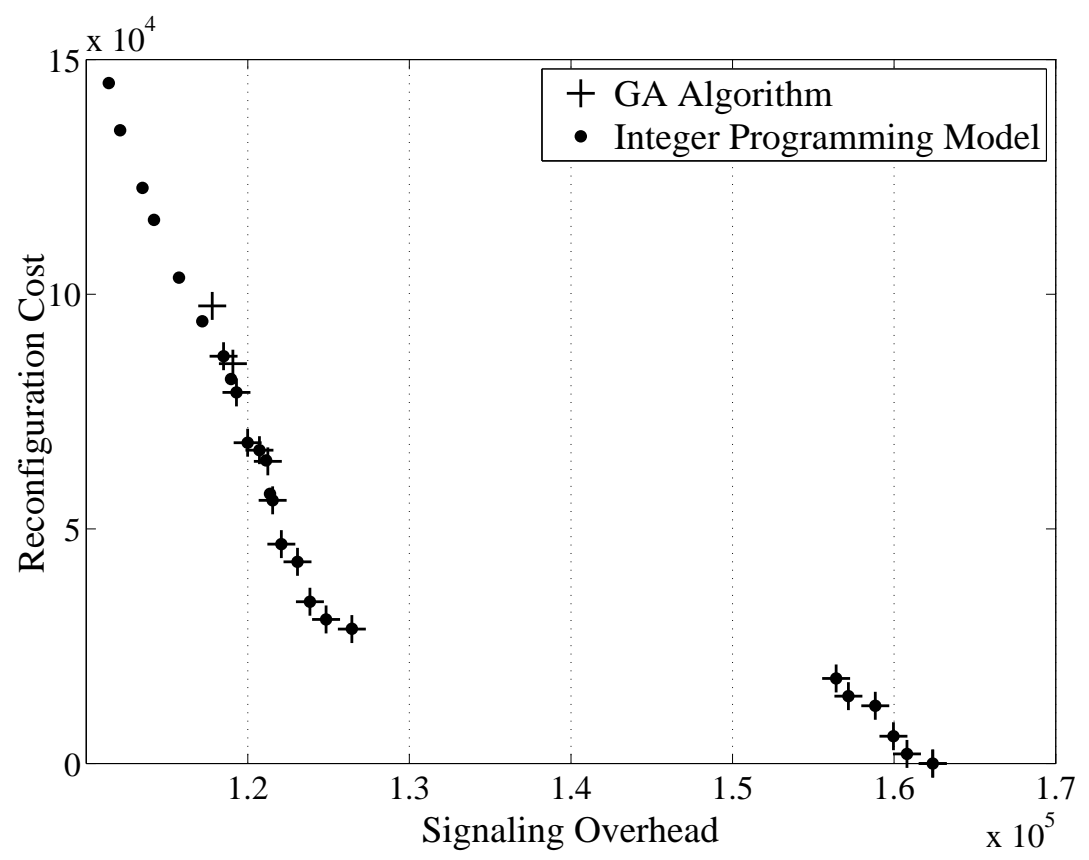

Figure 4.8: Pareto-optimal solutions of Network 1.

Table 4.2: Minimum-overhead solutions found by the two approaches.

\begin{tabular}{cccc}
\hline & $c_{S O}(\mathbf{t})$ & $c_{R}(\mathbf{t})$ & $\frac{c_{S O}\left(\mathbf{t}^{0}\right)-c_{S O}(\mathbf{t})}{c_{S O}\left(\mathbf{t}^{0}\right)}$ \\
\hline Integer Prog. Model & $1.1140 \times 10^{5}$ & $1.4499 \times 10^{5}$ & $31.40 \%$ \\
GA Algorithm & $1.1764 \times 10^{5}$ & $9.5504 \times 10^{4}$ & $27.84 \%$ \\
\hline
\end{tabular}

tained by the GA algorithm with the following parameters: POPSIZE $=100$, Iteration Limit $=10$ and PV-MAX $=20$. The PV matrix and the visited matrix are set to a size of 650-by-700, which gives a resolution of 255.18 and 249.83 units in overhead and cost, respectively. The GA computation took about 1 minute. The conclusions reached from this figure are as follows.

- By successively allowing higher reconfiguration cost, there is a jump in the improvement of signaling overhead. This shows the importance of approaching as many Pareto-optimal solutions as possible.

- The performance of the GA algorithm is close to optimality. It did not approach the point with the minimum overhead and highest recon- 


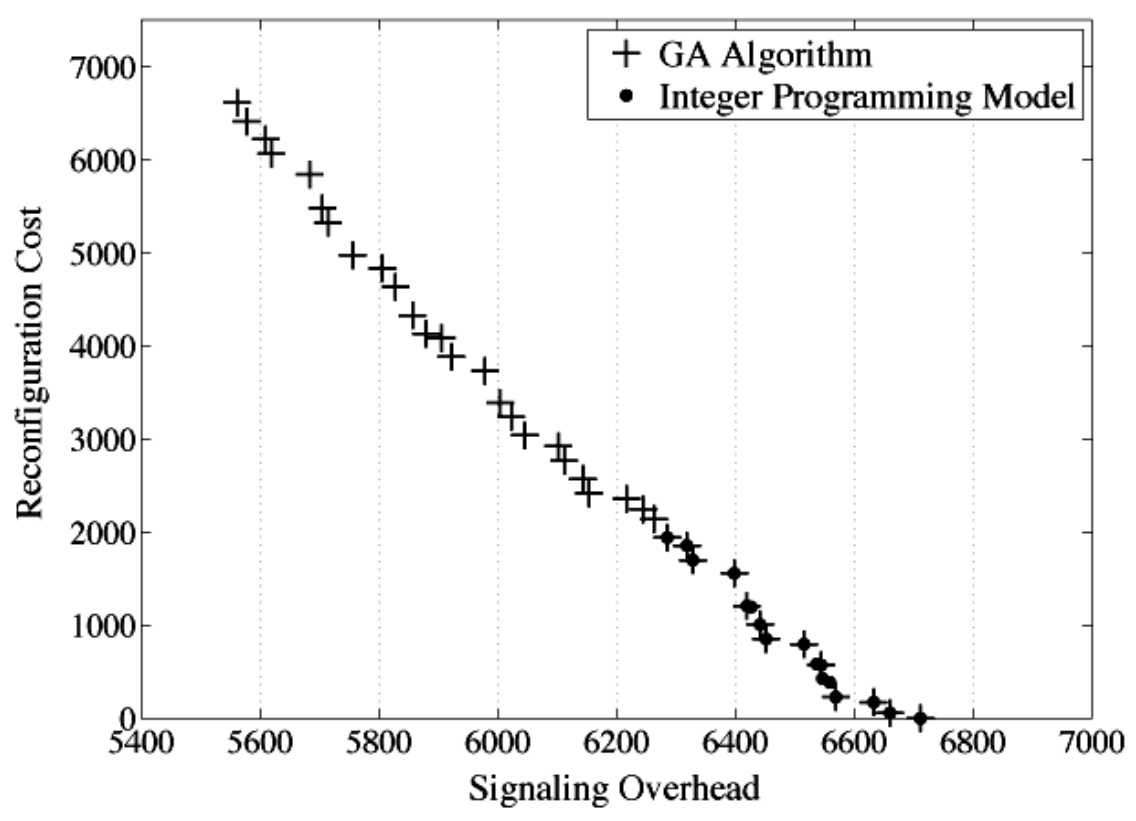

Figure 4.9: Pareto-optimal solutions of Network 2.

figuration cost. However, the relative performance difference is small. Table 4.2 compares the minimum-overhead solutions found by each approach for the Lisbon network. The overhead improvement of the integer programming model is $5.60 \%$ over the GA algorithm. Note that for achieving this extra improvement, the reconfiguration cost will increase by $51.81 \%$.

\subsubsection{Network 2}

The second data set represents a realistic deployment scenario for a network in one of the capital cities of Europe. The network consists of 75 sites and 225 cells. The optimum design for the reference scenario, $\mathbf{t}^{0}$, has twenty two TAs. Figure 4.9 shows the Pareto-optimal solutions of this network found by the two approaches. The integer programming model found some, but not all, of the exact Pareto-optimal solutions. It takes at least 1 hour to find each solution point. When B grows, the time required to find a solution increases rapidly to 8 hours. Therefore searching for exact Pareto-optimal 


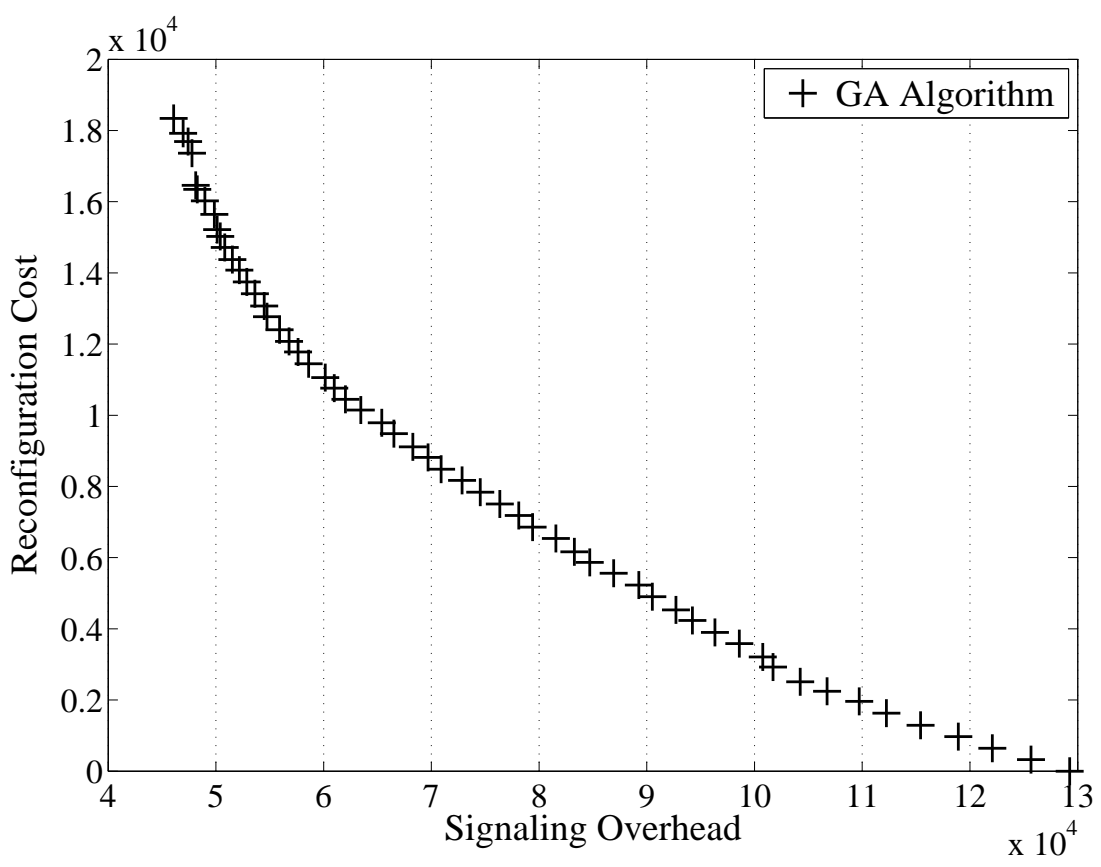

Figure 4.10: Pareto-optimal solutions of Network 3.

solutions for $B \geq 2000$ is not computationally feasible. To get the heuristic Pareto-optimal solutions from the GA algorithm in Figure 4.9, the following parameters are set: POPSIZE $=100$, Iteration Limit $=10$ and PV-MAX $=20$. The PV matrix is set to a size of 700-by-700, which gives a resolution of 10.55 and 9.59 units in overhead and cost, respectively. The GA computation took about 10 minutes. Below are the observations from the figure.

- The shape of the Pareto frontier, which is the set of all Pareto-optimal solutions of the signaling overhead and the reconfiguration cost, differs from that of network 1. The curve in Figure 4.9 is close to linear, meaning that to obtain improvement in overhead, the reconfiguration cost scales up proportionally.

- The exact Pareto-optimal solutions available from the integer programming model indicate that the Pareto-optimal solutions found by the GA algorithm are of very high quality. The GA algorithm performs very well and is time-efficient for this network. 


\subsubsection{Network 3}

The experiments for the third network use real-life data. The network is in use in an Asian capital city. The network consists of 339 sites and 978 cells. The number of defined TAs in the current configuration is six. For Network 3 , the computer memory needed by the solver exceeds what is available and it is therefore not possible to use the integer programming model. Figure 4.10 shows the Pareto-optimal solutions of this network obtained by the GA algorithm. The PV and visited matrices are set to a size of 1200-by-1400, which gives a resolution of 102.96 and 109.20 units in overhead and cost, respectively. It took 2 hours and 20 minutes for the GA algorithm to find the Pareto-optimal solutions in Figure 4.10 with the following parameters: POPSIZE $=300$, Iteration Limit $=3$ and PV-MAX $=20$. After the third iteration, no new Pareto-optimal solution was found. The observations from this figure are as follows.

- The smooth Pareto frontier indicates that the decision-maker has a large set of available trade-offs between the signaling overhead and the reconfiguration cost.

- The current TA configuration of the network is far from optimum in terms of signaling overhead. The Pareto-optimal solutions show that it is possible to decrease the overhead by $64 \%$. Figure 4.11 shows the initial TA design $\mathbf{t}^{0}$ of Network 3. Figure 4.12 illustrates the same network, when the Pareto-optimal solution with the lowest signaling overhead has been chosen. Each specific symbol in the two figures represents the sites inside one TA. In Figure 4.12, 111 sites have changed TAs in comparison to Figure 4.11 . Therefore, about $32 \%$ of the sites in the network are reconfigured. There are some elements in both figures that give the impression that the TAs are disjoint. The reason is the existence of highways which make direct handovers possible between those parts of the city.

\subsection{Conclusions}

A bi-objective optimization model has been presented for Pareto-optimal solutions for the trade-off between the signaling overhead and the TA reconfiguration cost. The proposed integer programming model provides the exact Pareto-optimal solutions, and the suggested GA algorithm is simple in implementation and efficient in performance for large-scale networks. The 
experiments on several real-life networks demonstrated that the characteristic of the Pareto frontier varies by network, and that the proposed GA algorithm provides close-to-optimal solutions for large-scale networks in feasible time. 


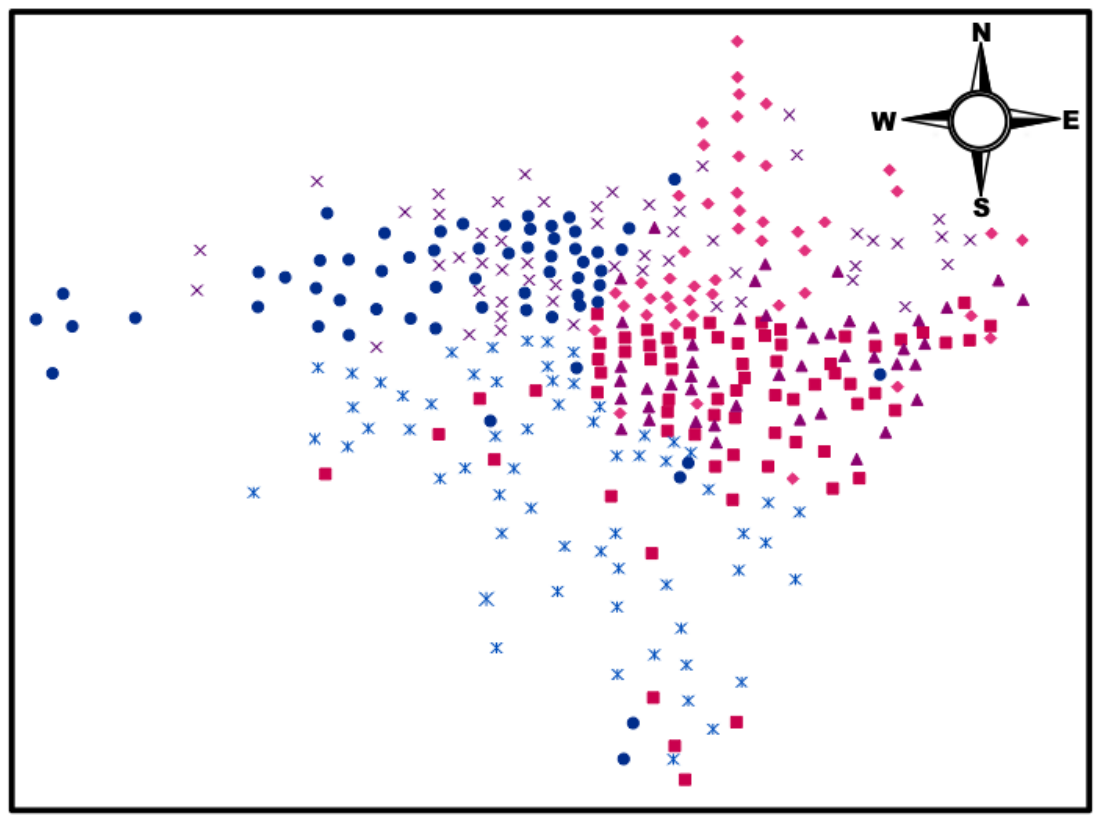

Figure 4.11: The initial TA design $\mathbf{t}^{0}$ of Network 3.

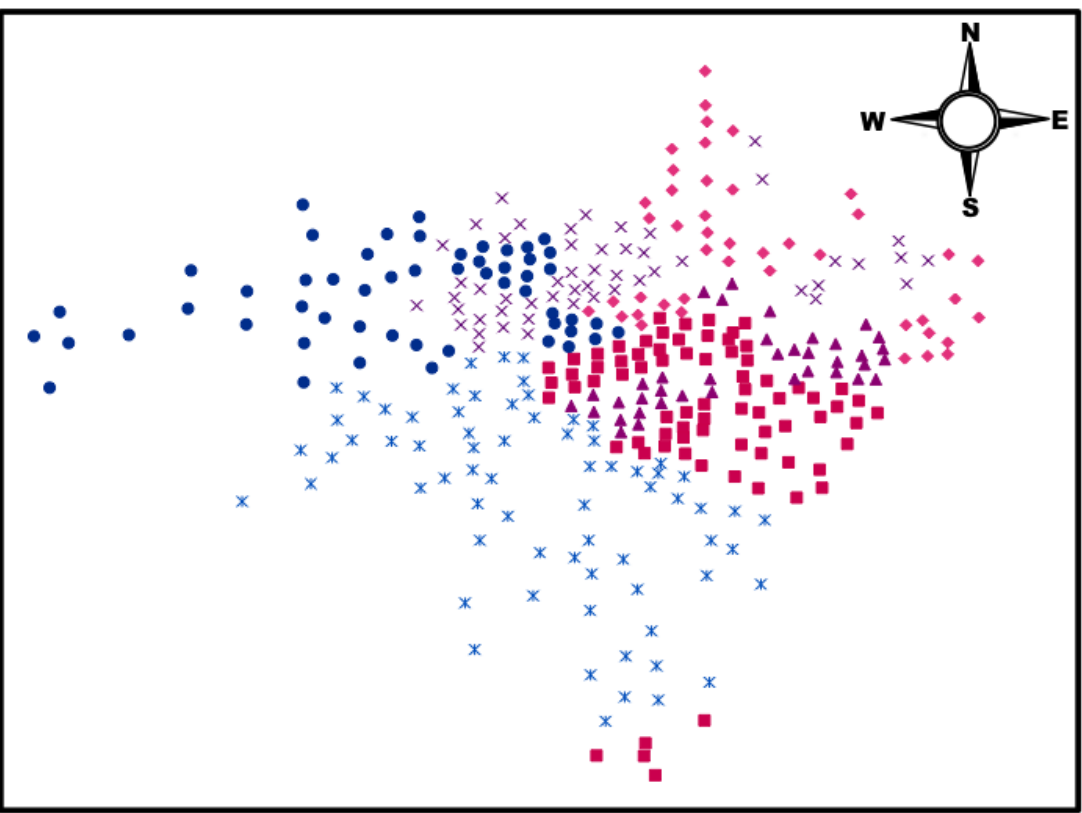

Figure 4.12: A Pareto-optimal solution of Network 3. 



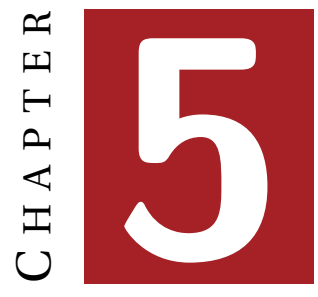

\section{Tracking AREA List}

In LTE, the cells can assign a list of TAs (i.e. Tracking Area List (TAL)) to each UE, and each cell can potentially assign different TALs to different UEs. This allows more flexible TA configuration and overcomes some of the limitations of the standard TA. Before studying the TAL scheme, it is instructive to consider the limitations of the standard TA scheme, which has been used in the previous chapters. It has already been suggested in the literature $[4,78]$ that the TAL scheme can prevent the frequent updates that arise when a UE keeps hopping between two or more adjacent cells in different TAs (the so called ping-pong effect). Further, TAL can solve the problem of high uplink traffic due to simultaneous updates of a large number of UEs crossing a TA boundary (the train scenario). In this chapter, in addition to these two problems, the symmetric and transitive properties of the standard TA scheme are compared with the flexible configuration of TAL. Later in the chapter, the challenges in applying the TAL scheme in a large scale network are explained.

This chapter aims to shed light on the idea of TAL, which is explored further in the coming chapters. Part of the discussion presented in this chapter has been published in [81].

\subsection{Limitations of Standard TA}

As has already been explained in this dissertation, in the standard TA scheme, cells are grouped into mutually disjoint sets, each being a TA. A cell belongs to exactly one TA, and each UE is registered to only one TA. The following subsections explain some well-known drawbacks of this scheme. 


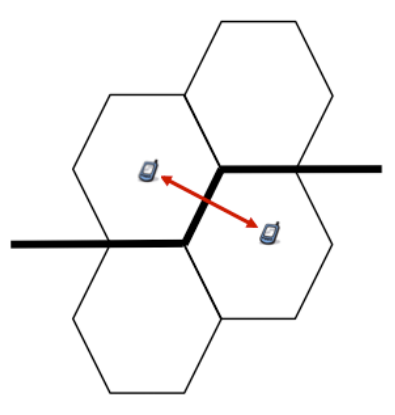

(a)

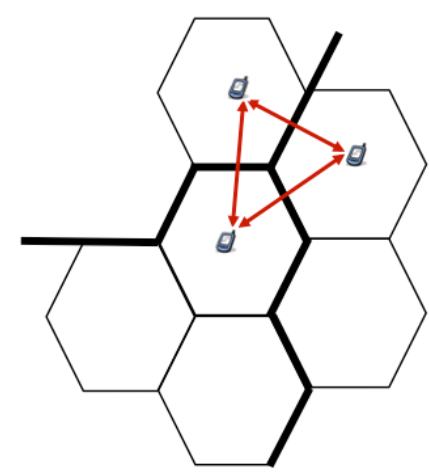

(b)

Figure 5.1: (a) ping-pong effect, (b) generalized ping-pong effect.

\subsubsection{Ping-Pong Effect}

It is often the case that UEs at the border of neighboring TAs move back and forth between the two or three neighboring TAs. These phenomena, shown in Figure 5.1, are referred to as the ping-pong and the generalized ping-pong effects, respectively [46]. Apart from the mobility of the UEs, fading of the radio channel can also cause the ping-pong effect. The effect causes excessive TAUs and accounts for a non-negligible portion of the total TAU signaling overhead.

In the standard TAU and paging scheme, no matter how the TAs are designed, the ping-pong effect exists between two neighbor TAs, and sometimes even between three neighbor TAs of a corner. In general, reducing the ping-pong effect can significantly improve the performance of a network and has therefore received considerable attention in the literature [36, 46, 70]. However, most of the proposed schemes for reducing this effect introduce a large paging overhead.

\subsubsection{Massive Mobility Signaling Congestion}

If a large number of UEs simultaneously move into a hotspot cell (e.g. UEs in a train arriving to a platform [78]), there is a risk of increased network load caused by excessive TAU from the UEs in a short period of time. Figure 5.2 shows the TAU storm, when a train passes a TA border. This is an undesirable situation from the network standpoint, as it could decrease the quality of service (QoS) in the cell and create signaling resource congestion [74]. 


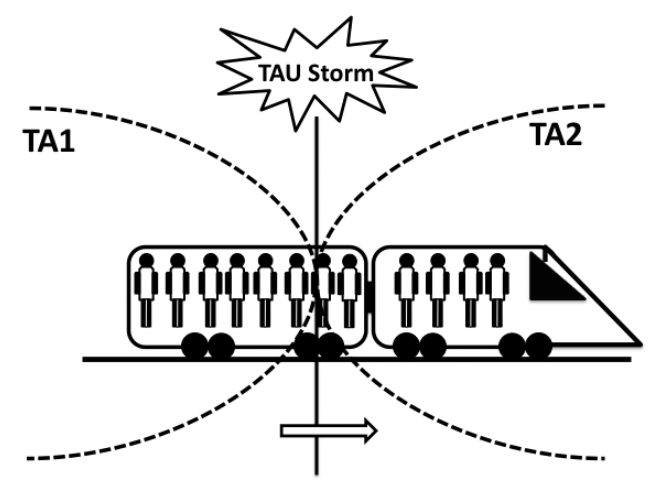

Figure 5.2: Example of TAU storm at the border of two TAs.

In addition to the train scenario, with the standard TA scheme, there are several types of mobility patterns that may cause TAU congestion. For example, in densely populated cities, there is a drastic difference in the mobility between day and night. A very large number of UEs moving concurrently into a central area (typically early morning of a weekday) may generate excessive signaling around the center $[3,74,78]$. Conversely, heavy paging may occur as a result of massive and almost static UEs simultaneously located at some hotspot (e.g., a large stadium). The problem of massive mobility signaling congestion is extensively considered and is presented in this dissertation in Chapters 8 and 9.

\subsubsection{Symmetric Property}

For the standard TA scheme, the following implication exists. If cell $A$ considers $B$ in the same TA, then cell $B$ also considers $A$ in the same TA. If cells could have different perspectives towards each other, then this flexibility might lead to a lower signaling overhead.

- Example: Consider $A$ and $B$ only from the network in Figure 5.3. The TA design either considers these two cells in the same TA, or separates them into two TAs. Based on (2.2), in the case that $A$ and $B$ are in the same TA, the signaling overhead is $c_{1}$ given in (5.1), and when $A$ and $B$ are in separate TAs the signaling overhead is $c_{2}$ given in (5.2). 


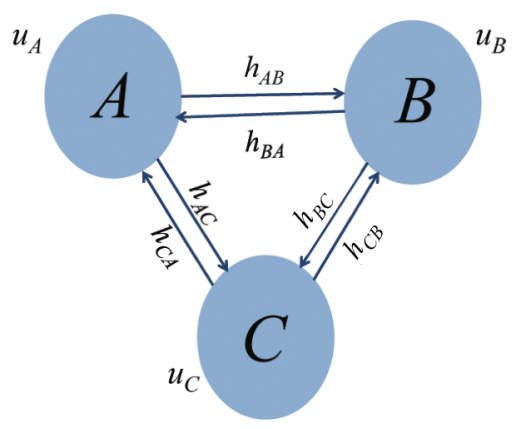

Figure 5.3: A three-cell network.

$$
\begin{array}{r}
c_{1}=\alpha c^{p}\left(u_{A}+u_{B}\right) \\
c_{2}=c^{u}\left(h_{A B}+h_{B A}\right) \\
c_{3}=c^{u} h_{A B}+\alpha c^{p} u_{B}
\end{array}
$$

Although it is not achievable by the standard TA scheme, let us consider a design in which all UEs in $A$ perceive that $B$ is in another TA but all UEs in $B$ assume $A$ is in the same TA. In this situation the signaling overhead is $c_{3}$ given in (5.3).

In conditions where $c_{1}-c_{3}>0$ and $c_{2}-c_{3}>0$ hold, corresponding to $\alpha c^{p} u_{A}>c^{u} h_{A B}$ and $c^{u} h_{B A}>\alpha c^{p} u_{B}$, the third assumption (5.3) leads to lower signaling overhead. Thus, when cell $A$ has high cell load, and there is no or few flow moving from cell $A$ to cell $B$, and $B$ has low cell load but high number of moves towards $A$, there is a potential to reduce the signaling overhead if the cells can break the symmetry in their view of TA.

\subsubsection{Transitive Property}

Another implication of the standard TA scheme is transitivity: if cells $A$ and $B$ are in the same TA, and cells $B$ and $C$ are in the same TA, then cells $A$ and $C$ must also be in the same TA.

- Example: Consider Figure 5.3 and two TA configurations for this network. The first configuration puts all three cells in one TA. Based on (2.1), this configuration results in matrix $\mathbf{S}_{1}(\mathbf{t})$ given in (5.4). In the 
second configuration, $A$ and $B$ are in the same TA, while $C$ forms its own TA, which results in matrix $\mathbf{S}_{2}(\mathbf{t})$ given in (5.5). The corresponding signaling overheads from (2.2), $c_{4}$ and $c_{5}$ are given in (5.6) and (5.7), respectively.

$$
\begin{gathered}
\mathbf{S}_{1}(\mathbf{t})=\left(\begin{array}{lll}
1 & 1 & 1 \\
1 & 1 & 1 \\
1 & 1 & 1
\end{array}\right) \\
\mathbf{S}_{2}(\mathbf{t})=\left(\begin{array}{lll}
1 & 1 & 0 \\
1 & 1 & 0 \\
0 & 0 & 1
\end{array}\right) \\
c_{5}=2 \alpha c^{p}\left(u_{A}+u_{B}+u_{C}\right) \\
c_{5}=\alpha c^{p}\left(u_{A}+u_{B}\right)+c^{u}\left(h_{A C}+h_{C A}+h_{B C}+h_{C B}\right)
\end{gathered}
$$

Let us assume that a design represented by matrix $\mathbf{S}_{3}(\mathbf{t})$ given in (5.8) can be created, disregarding the fact that it is not achievable using the standard TA scheme. The signaling overhead of matrix $\mathbf{S}_{3}(\mathbf{t})$ is equal to $c_{6}$ in (5.9).

$$
\begin{gathered}
\mathbf{S}_{3}(\mathbf{t})=\left(\begin{array}{lll}
1 & 1 & 0 \\
1 & 1 & 1 \\
0 & 1 & 1
\end{array}\right) \\
c_{6}=\alpha c^{p}\left(u_{A}+2 u_{B}+u_{C}\right)+c^{u}\left(h_{A C}+h_{C A}\right)
\end{gathered}
$$

In situations where $c_{4}-c_{6}>0$ and $c_{5}-c_{6}>0$, meaning that $\alpha c^{p}\left(u_{A}+u_{C}\right)>c^{u}\left(h_{A C}+h_{C A}\right)$ and $\alpha c^{p}\left(u_{B}+u_{C}\right)<c^{u}\left(h_{B C}+h_{C B}\right)$, designs (5.4) and (5.5) have higher signaling overhead compared to design (5.8). This example illustrates that there is a potential for reducing the signaling overhead if the transitivity condition can be relaxed.

Generally, in the standard TA scheme, there are three properties for any S(t) matrix: 
- $\mathbf{S}(\mathbf{t})$ is a binary matrix, where $s_{i j}(\mathbf{t})$ represents whether or not two cells are in the same TA.

- $\mathbf{S}(\mathbf{t})$ is a symmetric matrix, representing the obvious fact that if cells $i$ and $j$ are in the same TA, then cells $j$ and $i$ are also in the same TA (If $s_{i j}(\mathbf{t})=1$, then $\left.s_{j i}(\mathbf{t})=1\right)$.

- $\mathbf{S}(\mathbf{t})$ has the transitive property, meaning that whenever cells $i$ and $j$ are in the same TA, and cells $j$ and $k$ are in the same TA, then $i$ and $k$ are also in the same TA (If $s_{i j}(\mathbf{t})=s_{i k}(\mathbf{t})=1$ then $s_{j k}(\mathbf{t})=1$ ).

\subsection{Tracking Area List}

There have been extensive discussions in 3GPP regarding the preferred TA scheme for LTE systems. The standard TA scheme, which consists of nonoverlapping static TAs, as exists in GSM was used in earlier deployments. However, there are newer schemes that have the potential to avoid pingpong effects, distributing the TAU load more evenly across cells and reducing the overall TAU signaling overhead [88].

Introducing the concept of TAL gives more flexibility to the operators in their TA management. Some earlier candidate schemes that were discussed in the literature are as follows [91]:

- Overlapping TA: In this scheme, one cell is assigned to a list of overlapping TAs and a UE is assigned only to one TA of the cell's list. The UE does not perform a TAU while moving to a cell which has the assigned $\mathrm{TA}$ in its list.

- Multiple TAs: In this scheme, a cell belongs to only one TA, but a UE can be assigned more than one TA using a list. If one UE is assigned a list of TAs, the UE does not perform TAUs when it crosses the boundaries between these TAs. The TAs in this scheme are non-overlapping.

The more common scheme, which is also considered for TAL in this dissertation, is that a UE can be assigned a list of TAs, and a cell is able to assign different TALs to different UEs. A UE receives a TAL from a cell, and keeps it until it moves to a cell that is not included in its TAL. The location of a UE is known to the MME to the granularity of its TAL.

There are two general candidate architectures for the TAL assignment of LTE networks. One is to design TALs for UEs, meaning that the network 


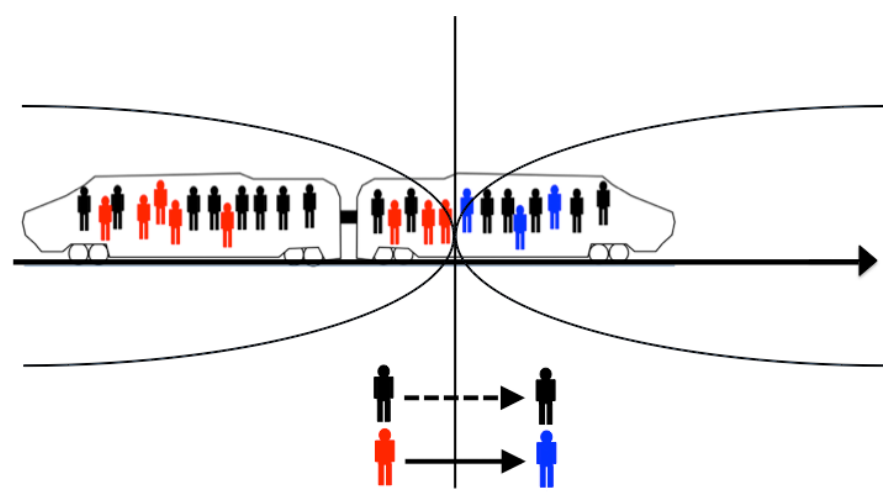

Figure 5.4: An example of reducing the signaling congestion by assigning different TALs to the UEs inside the train.

assigns each UE with a TAL proper to that UE's mobility behavior. One applicable solution here is to consider the previous TA in the updated TAL of the UE to avoid the ping-pong effect [5]. The other candidate architecture is to design TAL for cells based on the aggregated movement patterns and call arrival rates of the UEs. For example, to overcome the signaling congestion in the train scenario, the cells along the railway path can give different TALs to the UEs inside the train. Because the UEs inside the train are assigned different TALs, not all of them will perform TAU at the same time. In Figure 5.4, because the UEs in the train are given different TALs, only the ones which do not have the next TA in their TAL perform a TAU (red $\rightarrow$ blue). This significantly reduces the signaling congestion at TA borders.

It is not clear from the literature how to incorporate the individual and aggregated UE data in one algorithmic mechanism and to perform evaluation without the UE traces. Therefore, the former architecture is not considered here. Hence, although the combination of the two architectures introduces more flexibility in the system and may result in a better network performance, the latter architecture is considered as the fundamental of the studies further presented in this dissertation.

\subsection{Challenges in Applying TAL}

If a UE's location and mobility pattern were completely known, then the network could tailor a UE-specific TAL that virtually eliminates tracking signaling for the UE. However, in reality, the exact UE's future trajectories are not 


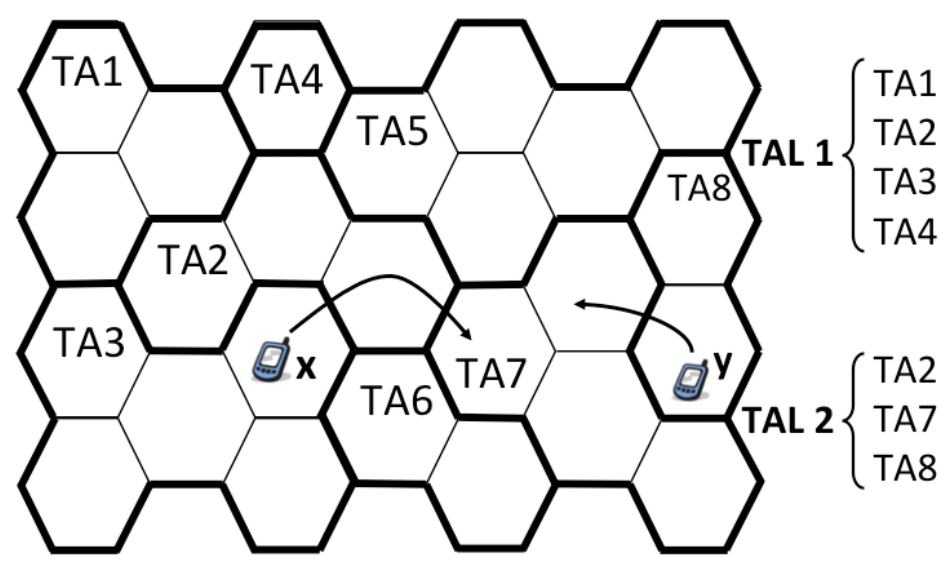

Figure 5.5: An example of TAL.

available, and models and algorithms for TAL configuration at a more aggregated level are required [20]. In the TAL schemes discussed in this dissertation, both UEs and cells are assigned a TAL. A UE receives a TAL from a cell, and keeps the TAL until it moves to a cell that is not included in any TA on the list. The UE location is known to the MME to the granularity of the TAL allocated to that UE.

- Example: In Figure 5.5, TAL1 consists of TA1, TA2, TA3 and TA4, and TAL2 consists of TA2, TA7 and TA8. Assume that the network gives TAL1 to UE $\mathbf{x}$ and TAL2 to UE $\mathbf{y}$. $\mathbf{x}$ will only make TAU when passing from TA2 to TA7, because $\mathbf{x}$ does not have TA7 in its TAL. $\mathbf{y}$ will not have any TAU while moving from TA8 to TA7. Note that if any of these UEs were paged, the paging message would be sent to all the cells inside the UE's TAL. Therefore, giving a proper TAL to each individual UE can be very beneficial in TAL design.

By using the TAL scheme, the UEs in one cell may have different TALs, depending on the cells from which the TALs are assigned. This difference of perspective makes the estimation of signaling overhead difficult. Figure 5.6 illustrates two UEs in cell $i$ moving towards cell $j$. The UEs are assigned different TALs which affect the TAU overhead calculation. If cell $j$ is included in the TAL of cell $i$, then no TAU overhead is needed for UEs having $i$ 's list and moving to cell $j$. This is the case for the upper UE in the figure. The UE below, on the other hand, does TAU because it does not have $j$ in its list. 


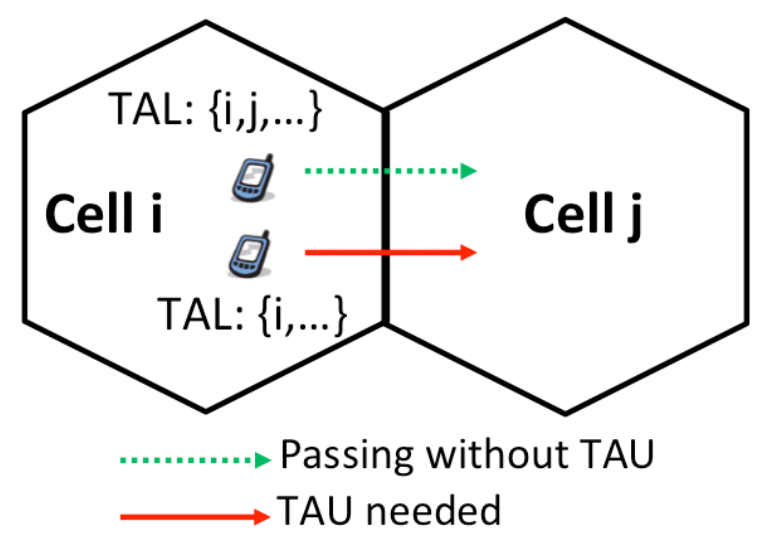

Figure 5.6: UEs are assigned to different TALs in one cell.

Similarly, UEs having TALs of other cells, such that the TALs have $i$ but not $j$, will generate TAU when moving from $i$ to $j$.

From the discussion above, it can be concluded that in designing TAL for a network, it is highly valuable to have accurate data traces of UEs' movements. Unfortunately, this data is either not available or too expensive to obtain. Also, designing TAL according to traces of UE mobility patterns limits the solutions to those specific movement sequences. If the UEs changed their movement behavior, which is quite probable, the TALs would become inefficient. 



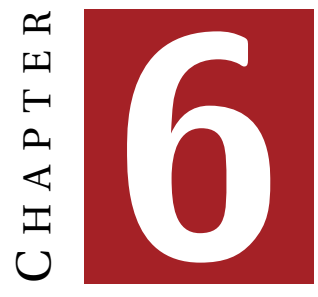

\section{Applying TAL in Cellular NETWORKS}

If the information of each individual UE's movement and calls were available to the network, then designing an optimum TAL would become trivial and could essentially result in the elimination of the signaling overhead. The network could give a specific, tailored list to each UE including all the cells the UE intends to pass before it will be called. This information, if available at all, is costly to obtain. Moreover, the validity of the information expires fast, because the UE trace is the history of the UE's movement, and the UE's intention of where and when to move in future is unknown.

Although TAL is expected to overcome some of the limitations of the standard TA scheme, how to optimize TAL in large-scale networks remains unexplored. This chapter proposes a signaling overhead calculation formula, which takes into account the discussion in Section 5.3. Three schemes are suggested in order to utilize TAL in large-scale networks. The advantages and disadvantages of each of these schemes are explained.

Here, the naturally arising questions are: What is the potential of reducing the signaling overhead in TAL in comparison to the standard TA scheme? Among all the TAL schemes, which one results in the lowest signaling overhead, and which one is the more practical one to be applied in a large-scale network? A carefully designed evaluation framework is presented to address these questions. This chapter is based on the work presented in [81, 82]. 


\subsection{Signaling Overhead Calculation for TAL}

To explore the flexibility of TAL, one can consider a TA as small as a single cell (i.e. no restriction on a given TA layout). Although LTE allows a cell to assign UEs different TALs, the assumption here is that a cell will give only one common TAL to all UEs being updated in that cell. Inevitably, this imposes restrictions on the performance of TAL.

Note that for the sake of reusing Equation (2.2), the notations $s_{i j}(\mathbf{t})$ and $\mathbf{S}(\mathbf{t})$ are kept. However, for the TAL design, the vector $\mathbf{t}$ does not exist and the TAL assignments can be defined in the form of an $N \times N$ matrix. For the TAL design, $s_{i j}(\mathbf{t})$ is defined in this dissertation as the proportion of UEs in cell $i$, who have $j$ in their TAL. Thus, the $\mathbf{S}(\mathbf{t})$ matrix would contain fractional values which are all between 0 and 1 . Although the matrix is no longer binary, Equation (2.2) remains valid. Given a TAL-cell assignment, there may be several ways to estimate $s_{i j}(\mathbf{t})$. In this section, first a one-hop estimation is considered, and then the idea is extended to two hops.

\subsubsection{One-hop Estimation}

The uncertainty factor in calculating $s_{i j}(\mathbf{t})$ stems from the fact that UEs in cell $i$ may be assigned to TALs of cells other than cell $i$. This happens because some UEs move to cell $i$, without updating to the TAL of $i$, because their TALs include $i$. To calculate $s_{i j}(\mathbf{t})$ in the one-hop estimation, the impact of neighbor cells of $i$ is considered. The formula is shown in (6.1). The denominator shows an estimation of the total number of UEs in $i$. The second term in the denominator calculates the number of UEs moving to $i$ without being updated by cell $i$, while $u_{i}$ estimates the number of UEs in cell $i$ having the TAL of $i$. The numerator estimates the number of UEs which are likely to have $j$ in their TALs. Parameter $l_{i j}$ is 1 if $j$ is in the TAL of $i$ and 0 otherwise. The neighbors of $i$ having $i$ in their TAL form the $Q_{i}$ set. Factor $\gamma$ represents the probability of UEs entering cell $i$ and having been updated in $Q_{i}$. The parameter $\gamma$ can be obtained from the statistical knowledge of a large population in the network.

- Example: In Figure 6.1 only the impact of neighbor cells of $i$ are considered, and the orange cells represent the neighbors containing $i$ in their TAL. If $l_{n_{1} i}=l_{n_{2} i}=1$, then $Q_{i}=\left\{n_{1}, n_{2}\right\}$. 


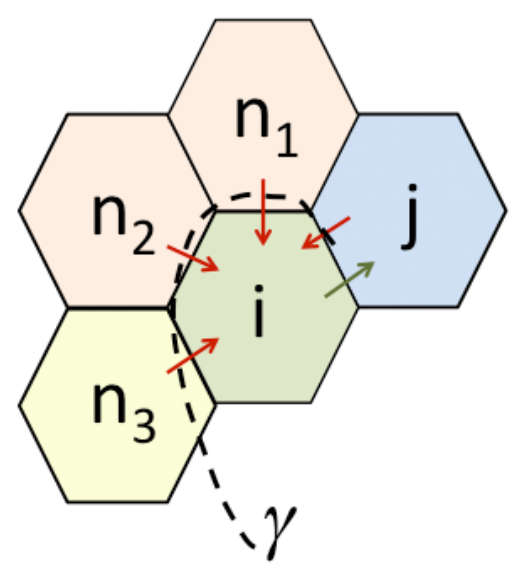

Figure 6.1: Parts of a network involved in one-hop estimation of the $s_{i j}(\mathbf{t})$.

$$
s_{i j}(\mathbf{t})=\frac{u_{i} l_{i j}+\gamma \sum_{n \in Q_{i}} h_{n i} l_{n j}}{u_{i}+\gamma \sum_{n \in Q_{i}} h_{n i}}
$$

The standard TA scheme can be used as a baseline for validating (6.1). For all $n \in Q_{i}$, because $Q_{i}$ is the set of neighbors of $i$ having $i$ in their TAL, $l_{n i}=1$. Also, for the standard TA scheme all the cells inside one TA are assumed to have the same TAL. If $l_{i j}=1$ then by the transitive relation in the standard TA scheme $l_{n j}=1$ for all $n \in Q_{i}$, and hence $s_{i j}(\mathbf{t})=1$. If $l_{i j}=0$ then again, due to transitivity, $l_{n j}=0$ for all $n \in Q_{i}$, and hence $s_{i j}(\mathbf{t})=0$. This leads to the conclusion that the parameter $\gamma$ does not play any role in the $s_{i j}(\mathbf{t})$ calculation of the standard TA scheme and $s_{i j}(\mathbf{t})=l_{i j}$, for all $i, j \in \mathscr{N}$.

Based on Equation (6.1), because the TAL of each cell contains that cell, the values on the diagonal of $\mathbf{S}(\mathbf{t})$ are always equal to one.

\subsubsection{Two-hops Estimation}

To extend the estimation to two hops, the impact of the neighbors of neighbors should be also included in the calculation. This increases the accuracy of the $\mathbf{S}(\mathbf{t})$ estimation. In Equation (6.2) two hops are considered. The denominator shows an estimation of the total number of UEs in $i$. The total number of UEs in cell $i$ is estimated by the sum of cell load of $i$ and the UEs entering $i$ with $i$ in their TAL. The numerator of (6.2) gives the number of 


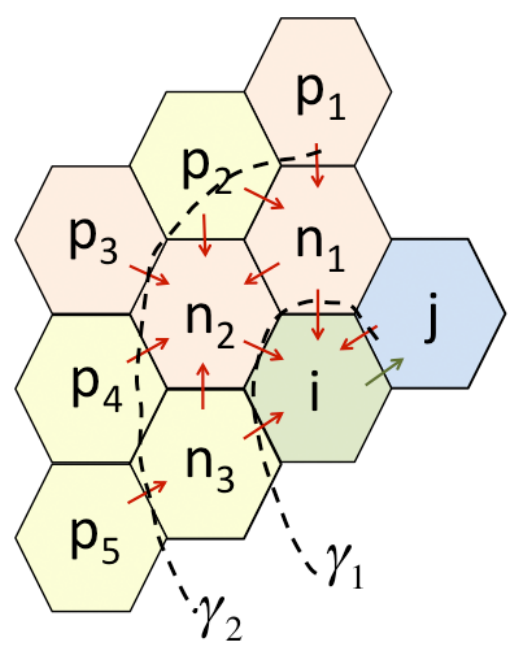

Figure 6.2: Parts of a network involved in two-hops estimation of the $s_{i j}(\mathbf{t})$.

UEs in $i$ estimated to have $j$ in their TALs. Parameter $l_{i j}$ is 1 if $j$ is in the TAL of $i$ and 0 otherwise.

$$
s_{i j}(\mathbf{t})=\frac{u_{i} l_{i j}+\gamma_{1} \sum_{n \in Q_{i}} h_{n i} l_{n j}+\gamma_{2} \sum_{n \in Q_{i}} \sum_{p \in Q_{n i}} \min \left(h_{p n}, h_{n i}\right) l_{p j}}{u_{i}+\gamma_{1} \sum_{n \in Q_{i}} h_{n i}+\gamma_{2} \sum_{n \in Q_{i}} \sum_{p \in Q_{n i}} \min \left(h_{p n}, h_{n i}\right)}
$$

As before, the neighbors of $i$ having $i$ in their TAL form the $Q_{i}$ set. Notation $Q_{n i}$ is the set of neighbors of $n \in Q_{i}$ having both $n$ and $i$ in their TALs. In most of the cases, $i \in Q_{n i}$, because as long as the neighborhood definition is defined in both directions, $i$ is considered as the neighbor of neighbor of $i$. While UEs move from the cells in $Q_{n i}$ to $n$ and thereafter to $i$, there will be no TAU.

- Example: In Figure 6.2 the impact of neighbors of neighbors is illustrated. The TAL of orange cells contain $i$ and the connected cells from the first-hop neighbors containing $i$. By assuming $l_{n_{1} i}=l_{i n_{1}}=l_{n_{2} i}=$ $l_{p_{1} n_{1}}=l_{p_{1} i}=l_{p_{1} n_{2}}=l_{p_{3} n_{2}}=l_{p_{3} i}=l_{n_{1} n_{2}}=1$ in the figure, one concludes $Q_{i}=\left\{n_{1}, n_{2}\right\}, Q_{n_{1} i}=\left\{i, p_{1}\right\}$, and $Q_{n_{2} i}=\left\{n_{1}, p_{1}, p_{3}\right\}$, and therefore the assigned TAL of UEs in cell $i$ can be the TAL of $i, n_{1}, n_{2}, p_{1}$ or $p_{3}$.

Factor $\gamma_{1}$ represents the probability of UEs entering cell $i$ having been updated in $Q_{i}$. Similarly, $\gamma_{2}$ is the fraction of UEs entering cell $i$ and have 
a TAL which is assigned by some cell in $Q_{n_{k} i}$ with $n_{k} \in Q_{i}$. The reason for picking the minimum value between $h_{p n}$ and $h_{n i}$ in the last term is to avoid overestimating the effects of UEs entering $i$.

It can be concluded from the definitions of $\gamma_{1}, \gamma_{2}$ and (6.2) that:

$$
\begin{array}{r}
\gamma_{1}+\gamma_{2} \leq 1 \\
\gamma_{2} \leq \gamma_{1}
\end{array}
$$

The constraints in (6.3) conclude that $\gamma_{2} \leq 0.5$. It should also be considered that not all combinations would be feasible. To give one example, $\gamma_{1}=\gamma_{2}=0.5$ is not a valid assumption, because it represents that for each cell, the impact of the second-hop cells (not including the cell itself) is as great as the first-hop cells.

\subsection{How to Design TAL?}

The aim is to find a scheme to design a TAL configuration for a cellular network using the available data. In this section, the following three schemes are introduced and explained in detail.

Scheme 1. TAL design independent from UE traces

Scheme 2. An intuitive rule of thumb

Scheme 3. TAL design based on UE traces

All the schemes are constructed based on the objective of improving the total signaling overhead.

\subsubsection{Scheme 1: TAL Design Independent from UE Traces}

For allocating and assigning TAL independently from UE traces, the only available information is the load of each cell and the number of handovers between cell pairs. In Section 6.1, simple formulas were defined for estimating all $s_{i j}(\mathbf{t})$ values of the $\mathbf{S}(\mathbf{t})$ matrix, considering one or two hops. The definition of the $l_{i j}$ parameter in the formulas requires a TAL assignment. Thus, the problem is narrowed down to the challenge of finding a TAL assignment resulting in an estimated $\mathbf{S}(\mathbf{t})$ matrix which improves the total signaling overhead calculated by Equation (2.2).

Because UEs in one cell can be assigned different TALs, it is clearly not possible to set each element of $\mathbf{S}(\mathbf{t})$ completely independently from the other 


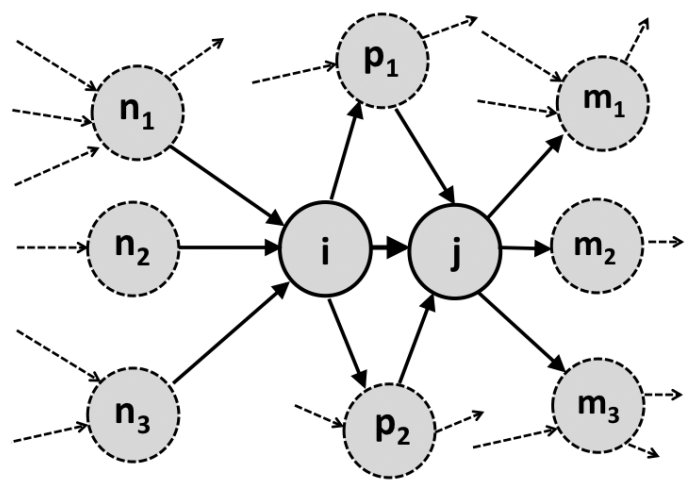

Figure 6.3: An example of the dependency between elements of $\mathbf{S}(\mathbf{t})$.

elements. Also, it has already been mentioned in Section 6.1 that the $s_{i j}(\mathbf{t})$ values in the TAL can be fractional.

- Example: One can observe from Figure 6.3 that by adding or omitting $j$ from the TAL of $i$, not only $s_{i j}(\mathbf{t})$, but $s_{p_{1} j}(\mathbf{t}), s_{p_{2} j}(\mathbf{t}), s_{j m_{1}}(\mathbf{t}), s_{j m_{2}}(\mathbf{t})$ and $s_{j m_{3}}(\mathbf{t})$ can all change in the $\mathbf{S}(\mathbf{t})$ matrix.

The following local search algorithm is developed to make the TAL assignment of each cell, considering the effects on the other cells.

\subsubsection{Local Search Algorithm}

The algorithm iteratively updates the TAL assignment of each cell. The basic operation of the algorithm is to modify the TAL of one cell at a time, by either deleting or adding one of the elements in the TAL. Then, depending on whether one or two hops are used in the algorithm, Equation (6.1) or (6.2) is used respectively to estimate the $\mathbf{S}(\mathbf{t})$ matrix, which in turn gives the total signaling overhead. This is repeated until no additional change results in any improvement.

The local search algorithm is formalized in Algorithm 3, in which the initial solution given to and returned by the algorithm is denoted by $\mathrm{tal}^{l}$, and the algorithm considers one-hop calculation. The optimal standard TA configuration can be used as the starting TAL assignment of the network. This means that the cells which belong to the same TA are given a list containing all the cells in the TA. Of course, with this solution, the $\mathbf{S}(\mathbf{t})$ matrix is binary, 


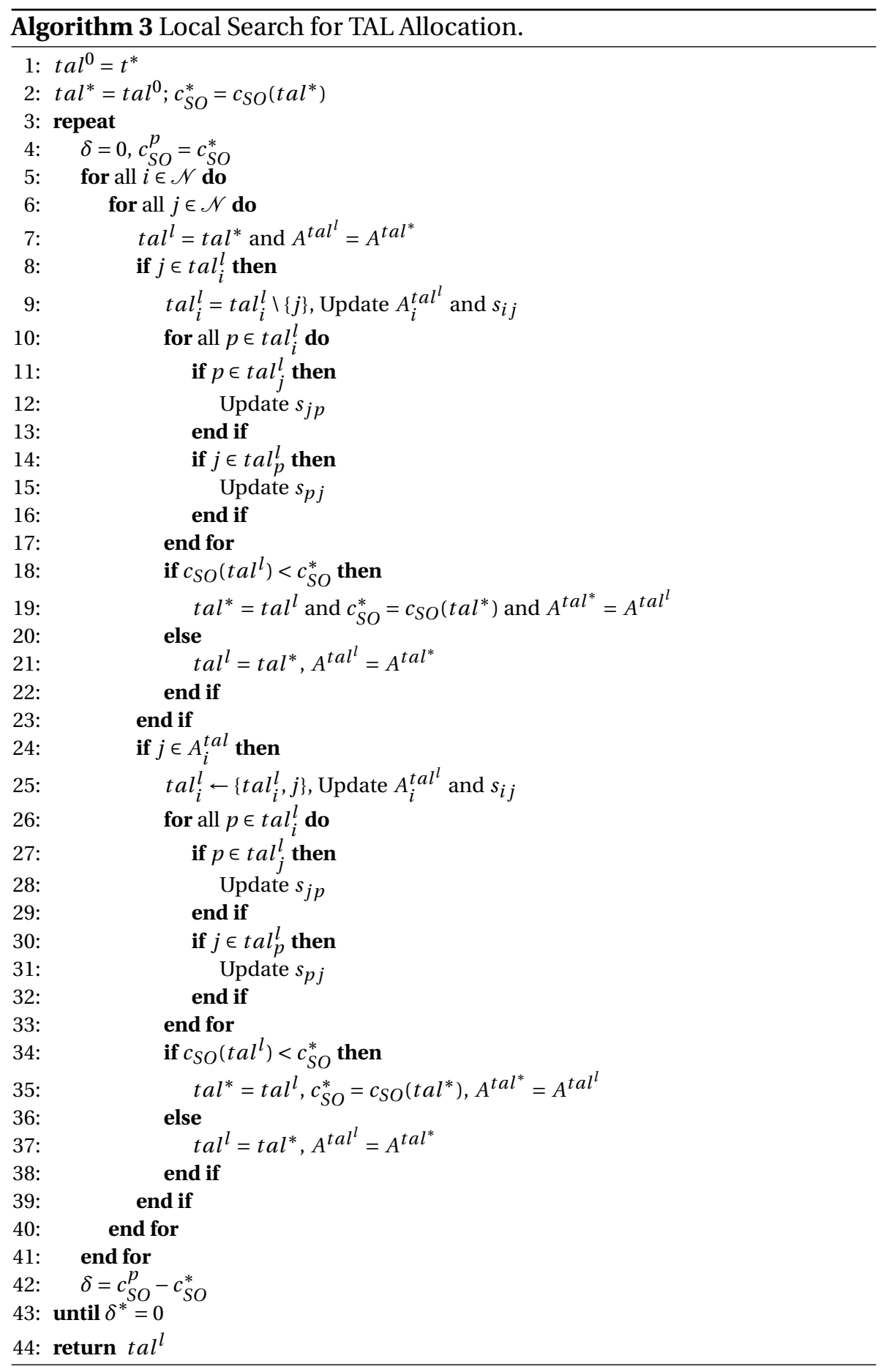


and is by definition, equal to the matrix obtained from the condition stated in (2.1).

At line 8, the algorithm checks whether or not $j$ should be in the TAL of $i$. If $j$ belongs to the list of $i$, the algorithm removes this cell at line 9 , and if it does not belong to the list of $i$, but it is adjacent to the TAL of $i$, the algorithm adds it to the list of $i$ at line 25. All the neighbor cells to the TAL of $i$ are stored in $A_{i}^{t a l}$. When $j$ is added or removed from the TAL of $i$, row $A_{i}^{\text {tal }}$ of the matrix $A^{\text {tal }}$ is updated by the algorithm.

According to the discussion in Section 6.2.1 and Figure 6.3, it can be concluded that by adding/removing only one cell $j$ to/from the TAL of another cell $i$, there are three elements in the $\mathbf{S}(\mathbf{t})$ matrix which should be updated, in the case of one-hop calculation:

$$
\begin{aligned}
& \cdot \mathrm{s}_{i j}(\mathbf{t}) \\
& \cdot \mathrm{s}_{p j}(\mathbf{t}) \text { for all } p \in t a l_{i} \cap t a l_{j} \\
& \cdot \mathrm{s}_{j p}(\mathbf{t}) \text { for all } p \in t a l_{i} \cap t a l_{p}
\end{aligned}
$$

The algorithm changes these elements of $\mathbf{S}(\mathbf{t})$ and calculates the signaling overhead. If the change results in a lower signaling overhead, then the change is kept; if it results in a higher signaling overhead, the previous configuration is again active, and the change is discarded. By repeating this procedure for every $(i, j)$ pair, a modified TAL will be defined for each cell. The algorithm iterates until no more improvement is possible. Note that the algorithm does not achieve the optimum TAL design, but rather aims towards an improved configuration which should result in a lower total signaling overhead compared to the standard TA scheme.

\subsubsection{Advantages}

- The scheme does not require any information regarding the UE traces. The same input data for designing a standard TA scheme can also be used here.

- The scheme considers the impact of adding or omitting a cell from the list of other cells on either one-hop or two-hops cells; therefore the calculation tends to take care of the challenge explained in Section 5.3. 


\subsubsection{Disadvantages}

- The scheme is based on the $s_{i j}(\mathbf{t})$ formulas suggested in (6.1) and (6.2). Each cell has its own value of $\gamma$. However, the average estimation of this value over the network is considered in the formulations. It is hard to approach a good average estimation of $\gamma$, which influences the TAL design and the resulting signaling overhead. In the two-hops formula, a good estimation of the ratio between $\gamma_{1}$ and $\gamma_{2}$ is also important.

- Applying the scheme by considering two-hops formulation has a rather high complexity of calculations.

- The starting point in the algorithm of the scheme has an impact on the final result. A reasonable available starting point is to use the optimum standard TA configuration. This requires obtaining the optimum standard TA design before starting the algorithm.

\subsubsection{Scheme 2: An Intuitive Rule of Thumb}

The flexibility of TAL compared to the standard TA scheme is that $\mathbf{S}(\mathbf{t})$ is not necessarily binary nor symmetric. If one considers a network with only two cells (e.g. cells $i$ and $j$ in Figure 6.1 and ignoring the rest). There are two choices for designing the TAL of cell $i$ :

1. $\operatorname{tal}_{i}=\{i\}$

In this case, the signaling overhead resulting from cell $i$ is $c^{u} h_{i j}$. This means that all the flow moving from $i$ to $j$ should have a TAU, because $j$ is not included in the TAL of $i$.

2. $\operatorname{tal}_{i}=\{i, j\}$

Here, the signaling overhead resulting from cell $i$ is $\alpha c^{p} u_{i}$, meaning that if a UE is paged in $i$, there will be paging in cell $j$, but there is no TAU for the UEs moving from $i$ to $j$.

Thus, in order to minimize the signaling overhead resulting from cell $i$, the following decision can be made:

$$
l_{i j}= \begin{cases}1 & \text { if } \alpha c^{p} u_{i}<c^{u} h_{i j} \\ 0 & \text { otherwise }\end{cases}
$$

If $\alpha c^{p} u_{i}<c^{u} h_{i j}$, then it is desirable for $i$ to include $j$ in its TAL. The same logic can be applied for designing the TAL of cell $j$. This principle is 
generalized to the whole network by separately considering each $(i, j)$ pair in the network.

\subsubsection{Advantages}

- The scheme is simple and easy to apply on a large-scale network. It often gives a good TAL assignment. That is why it is named a rule of thumb for designing TAL.

- The scheme does not require any information other than the load and handover of the cells. It does not even depend on the standard TA configuration or tuning of any parameter.

- The scheme has very low computational complexity.

\subsubsection{Disadvantages}

- The main disadvantage of the scheme is that it only searches among the one-hop neighbors of a cell to be included in the TAL of that cell. Thus, the maximum length of a cell's TAL is the number of neighbors of the cell plus one. This limitation becomes critical if reducing the TAU overhead is prioritized over reducing the paging overhead (i.e. $\left.c^{u} \gg c^{p}\right)$.

- In this scheme, each cell "selfishly" optimizes the signaling overhead according to its own data and does not consider the joint effect of the other cells' TALs.

\subsubsection{Scheme 3: TAL Design based on UE Traces}

In the previous two schemes, the TAL design has been based on the load and handover of cells for a time duration. If possible, it would be desirable to utilize UE traces in designing a TAL. With the earlier cellular technologies, getting the traces of idle UEs was an extremely costly and unfeasible procedure. However in LTE, there are some possibilities for collecting a small portion of UE traces. One is the existence of UEs using GPS-featured applications in the network. Another possibility is to apply the TAL scheme, which is explained below and which collects a set of UE traces. 


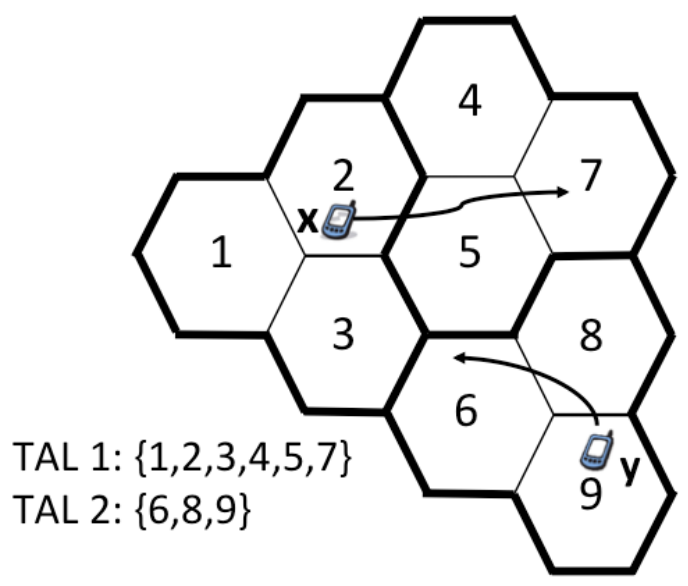

Figure 6.4: An example of how to collect part of UE traces.

\subsubsection{Collecting UE traces by the TAL scheme}

Since a cell is able to give different TALs to different UEs, it becomes feasible to collect a fraction of UE traces. If all cells in a network update a specific UE by giving it a TAL which only contains the updating cell, then the network can trace that specific UE. The idea is clarified by the following example.

- Example: In Figure 6.4, first let us consider that $\mathbf{x}$ and $\mathbf{y}$ follow the general configuration of the network, and are assigned to TAL1 and TAL2, respectively. In this case, none of the UEs will perform an update for the UE trace shown in the figure. In the next step, consider that both $\mathbf{x}$ and $\mathbf{y}$ are two specific UEs, for which the network collects their traces. In this case, every cell gives TAL of a single cell to $\mathbf{x}$ and $\mathbf{y}$, and not TAL1 and TAL2. The TALs of $\mathbf{x}$ and $\mathbf{y}$ always consist of one cell, which is the current serving cell. Therefore, by all the updates, the trace 2-5-7 can be collected for $\mathbf{x}$ and the trace 9-8-6 can be collected for $\mathbf{y}$.

The price of collecting a portion of UE traces is the number of updates that the selected UEs create. Note that for these selected UEs, there is no paging overhead.

\subsubsection{Optimization Algorithm}

Unlike the other two schemes, the load and handover of the cells are not used in the optimization algorithm of this scheme. Instead, a portion of UE 
traces is considered as the input data. The algorithm goes through these UE traces and considers whether adding or omitting a cell from the TAL of another cell will potentially improve the total signaling overhead or not. The algorithm uses the same basic TAL-modification operation as in Algorithm 3, explained in the first scheme. The difference is that the collected UE traces are used in the signaling overhead estimation. The result is the optimized TA assignment for the available UE traces, and the solution can be applied to the entire network. Of course, having $100 \%$ of the UE traces, could lead to a close-to-optimum TAL assignment for the network.

\subsubsection{Advantage}

- The scheme takes advantage of the tracing possibility allowed by the flexibility of TAL.

\subsubsection{Disadvantage}

- In order to obtain a reasonable result for this scheme, the network has to trace some UEs at the accuracy of the cell level. This may cause too much signaling if the sampling percentage is large.

\subsection{Performance Evaluation of the Proposed TAL Schemes}

The performance of a TAL scheme is always dependent on the UE traces inside the network. In order to have a fair comparison between the standard TA and the proposed TAL schemes, all the schemes are applied to the same UE-traces scenario. A UE-traces scenario is a set of UE traces and call arrivals for a specific time period in a network, matching the cell load and handover. One set of cell load and handover data, can have many matching UE-traces scenarios. The UE-traces scenario is dependent on the chosen mobility model, including the amount and speed of each UE's movement.

There is an extensive amount of research on mobility models using analytical and simulation approaches [27, 33, 34]. However, the study of UEs mobility behaviors is out of the scope of this dissertation, and hence the methods applied for generating the UE-traces scenario used for the performance evaluation, and aggregating the data from it, are explained in Appendix B. 
The standard TA and TAL schemes can be applied to the same UE-traces scenario and their performance can be compared to each other. For most of the schemes, however, the aggregated data in load and handover are the input. One method for the accurate calculation of the total signaling overhead is to directly count the total number of TAU and paging signalings in the UE-traces scenario.

\subsubsection{Signaling Overhead Calculation}

Assuming that each cell has received the TAL of the starting cell, the TAL of each UE is known in all the time intervals through following the UE trace. Each time a UE moves from a cell which is not included in the UE's TAL, the TAU cost $c^{u}$ is added to the total signaling overhead. Depending on the length of the TAL, denoted by $L_{t a l}$, at the time of paging, $\left(L_{t a l}-1\right) \times c^{p}$ is added to the total signaling overhead. When the TAL of a UE consists of only the serving cell, the paging overhead for the UE is zero. Similarly, in longer TALs, the serving cell should be excluded from the paging overhead. This is the reason why the value one is reduced from $L_{t a l}$ in the paging overhead computation. This procedure is repeated for all the UEs in the UE-traces scenario. Thus, the obtained total signaling overhead is the exact value for that UE-traces scenario.

\subsection{Numerical Results}

The numerical study that follows is based on the data from the Lisbon network. Ten UE-traces scenarios were generated by using the method in B.1 which resemble one available set of data of the cell load and handover in one hour. The number of UEs in all UE-traces scenarios is 25,000 . The one-hour time period is divided into 60 equal time intervals. Thus, the time granularity is 1 minute.

The dimension of the scenario matrices in all ten scenarios is $25,000 \times$ 60 . The parameter $\alpha$ is 0.0167 , which means $1.67 \%$ of the UEs are paged in the UE-traces scenarios. The possibility of a UE being called several times during the one hour time period is also considered. This is done by allowing the UE which was called previously to enter the random call selection for its other time slots. Among the elements of the scenario matrix, the number of active UEs scaled by the time proportion is $5 \%$ of the total cell load. While a UE is in the active state, there is no signaling overhead for that UE during the call. The average length of the call durations is assumed to be 3 minutes. 
Table 6.1 gives the numerical assumptions and values for this performance evaluation.

The cell load and handover data are aggregated from each UE-traces scenario, based on the method explained in B.2. The aggregated cell load and handover are comparable to those in the original data. The assumptions are that $c^{u}=1$ and $c^{p}=0.1$. The optimum standard TA configuration is computed by CPLEX [60] using the model in [109] for each UE-traces scenario data set. TAL configuration is at the granularity of the cell level.

Table 6.1: Numerical assumptions and values for performance evaluation.

\begin{tabular}{lll}
\hline Parameter & Description & Values \\
$\alpha$ & Call intensity factor & 0.0167 \\
$c^{p}$ & Cost of a single paging & 0.1 \\
$c^{u}$ & Cost of a single TAU & 1 \\
$N$ & Number of cells & 164 \\
$T$ & Number of TAs & {$[11-30]$} \\
\hline
\end{tabular}

The three schemes presented earlier in this chapter for designing TAL are implemented in MATLAB. The computations are run on an Intel Core 2 Duo of $2.1 \mathrm{GHz}$.

\subsubsection{Standard TA Design}

The signaling overhead of the standard TA (STA) design can be simply computed from the aggregated cell load and handover. As a baseline for the performance evaluation, the signaling overheads of the STA design were computed for the ten UE-traces scenarios based on the method explained in Section 6.3. The results are presented in Table 6.2. The values for all UE-traces scenarios are very close, because all are based on the same set of original cell load and handover data.

\subsubsection{Scheme 1: Trace-independent TAL Design}

A TAL design based on Scheme 1, which was presented in Section 6.2.1 and considers the two-hops estimation, was designed for each UE-traces scenario. The local search algorithm took on average 4 minutes to design TAL for one UE-traces scenario. The signaling overheads for this TAL design were calculated based on 6.3 for all ten UE-traces scenarios. The results are presented in Table 6.3. The observations from comparing the tables can be summarized as follows: 
Table 6.2: Signaling overheads of the STA design.

\begin{tabular}{c|rrr}
\hline & \multicolumn{3}{|c}{ STA } \\
\hline Scenario No. & TAU & Paging & Total \\
\hline 1 & 421 & 598.7 & 1019.7 \\
2 & 428 & 549.6 & 977.6 \\
3 & 382 & 607.2 & 989.2 \\
4 & 449 & 498.9 & 947.9 \\
5 & 357 & 622.3 & 979.3 \\
6 & 331 & 616.1 & 947.1 \\
7 & 325 & 622.6 & 947.6 \\
8 & 483 & 494.4 & 977.4 \\
9 & 382 & 620.4 & 1002.4 \\
10 & 363 & 624.9 & 987.9 \\
\hline
\end{tabular}

Table 6.3: Signaling overheads of the TAL design obtained from Scheme 1.

\begin{tabular}{c|ccc}
\hline & \multicolumn{3}{|c}{ TAL1 } \\
\hline Scenario No. & TAU & Paging & Total \\
\hline 1 & 71.0 & 348.8 & 419.8 \\
2 & 49.0 & 382.4 & 431.4 \\
3 & 64.0 & 382.4 & 446.4 \\
4 & 64.0 & 340.2 & 404.2 \\
5 & 48.0 & 380.2 & 428.2 \\
6 & 43.0 & 372.8 & 415.8 \\
7 & 36.0 & 366.2 & 402.2 \\
8 & 49.0 & 356.5 & 405.5 \\
9 & 64.0 & 381.1 & 445.1 \\
10 & 33.0 & 375.2 & 408.2 \\
\hline
\end{tabular}

- The total signaling overheads from the TAL designs are $54 \%$ to $58 \%$ better than those obtained from the optimal STA designs.

- The TAL algorithm significantly reduces the TAU overhead in relation to the paging overhead. The TAU overheads are reduced by $83 \%$ to $90 \%$ compared to the values obtained from the STA design. 
Table 6.4: Signaling overheads of the TAL design obtained from Scheme 2.

\begin{tabular}{c|ccc}
\hline & \multicolumn{3}{|c}{ TAL2 } \\
\hline Scenario No. & TAU & Paging & Total \\
\hline 1 & 317 & 160.0 & 477.0 \\
2 & 295 & 162.4 & 457.4 \\
3 & 257 & 163.6 & 438.6 \\
4 & 312 & 162.9 & 474.9 \\
5 & 274 & 165.0 & 439.0 \\
6 & 267 & 163.0 & 430.0 \\
7 & 285 & 162.7 & 447.7 \\
8 & 268 & 157.1 & 425.1 \\
9 & 306 & 162.8 & 468.8 \\
10 & 306 & 163.9 & 469.9 \\
\hline
\end{tabular}

\subsubsection{Scheme 2: TAL Design based on Rule of Thumb}

A TAL design based on the rule of thumb in Section 6.2.2 was designed for each UE-traces scenario. It took around 10 seconds to design TAL for one UE-traces scenario. The results of the signaling overhead values are given for the ten UE-traces scenarios in Table 6.4. The observations from comparing the tables can be summarized as follows:

- The total signaling overheads from the TAL design of Scheme 2 are $49 \%$ to $56 \%$ better than those of the optimal STA designs.

- The rule of thumb in designing TAL significantly reduces the paging overhead in relation to the TAU overhead. The paging overheads are reduced by $67 \%$ to $73 \%$ compared to the values obtained from the STA design. This is to be expected because the rule of thumb tends to create small TALs.

- The total signaling overheads obtained from Scheme 2 are $2 \%$ to $15 \%$ lower than those obtained from Scheme 1. The exception is Scenario No. 3 which resulted in $5 \%$ lower total signaling overhead. To compare the two schemes, one should also consider the speed in reaching these results. 
Table 6.5: Signaling overheads of STA and TAL3 designs for 1250 UEs.

\begin{tabular}{c|ccc|ccr|c}
\hline & \multicolumn{3}{|c|}{ STA } & \multicolumn{3}{c|}{ TAL3 } & Cost \\
\hline Scenario No. & TAU & Paging & Total & TAU & Paging & Total & TAU \\
\hline 1 & 29 & 29.8 & 58.8 & 1 & 4.2 & 5.2 & 389 \\
2 & 25 & 30.3 & 55.3 & 0 & 5.9 & 5.9 & 355 \\
3 & 19 & 30.1 & 49.1 & 1 & 6.6 & 7.6 & 359 \\
4 & 19 & 34.3 & 53.3 & 3 & 8.0 & 12.0 & 365 \\
5 & 13 & 27.7 & 40.7 & 3 & 4.8 & 7.8 & 368 \\
6 & 17 & 35.3 & 52.3 & 4 & 7.2 & 11.2 & 351 \\
7 & 28 & 28.2 & 56.2 & 0 & 7.1 & 7.1 & 355 \\
8 & 25 & 19.2 & 44.2 & 4 & 5.9 & 9.9 & 383 \\
9 & 22 & 14.5 & 36.5 & 1 & 5.5 & 6.5 & 355 \\
10 & 21 & 33.5 & 54.5 & 2 & 7.0 & 9.0 & 387 \\
\hline
\end{tabular}

\subsubsection{Scheme 3: TAL Design based on UE Traces}

An optimized TAL design based on 5\% of the total number of UE-traces was designed for each UE-traces scenario. It took around 2 hours for the algorithm to design the TAL for the 1250 UE traces. For these specific UEs, the signaling overheads obtained from the STA design and the TAL design based on UE traces (named TAL3) are given in Table 6.5. The significant reduction in the signaling overheads for TAL3 shows the scheme has a potential in comparison to the STA scheme.

The last column of Table 6.5 gives the cost of collecting these 1250 UE traces. Recalling the discussion in Section 6.2.3, all these 1250 UEs are given TALs which only contain the updating cell. Thus, there will be no paging overhead, and the cost of UE-trace collection is the amount of TAU.

For each scenario, TAL3 is applied to the entire UE-traces scenario. Table 6.6 presents both the signaling overheads of Scheme 3 without considering the cost of collecting data, and those where this cost is added. The observations from this table and Table 6.5 can be summarized as follows:

- While not adding the cost, the signaling overheads in Table 6.6 show that except for one scenario (Scenario No. 8), there is a reduction in the total signaling overhead. However, the improvement is much smaller in comparison to the results of the previous two TAL schemes.

- Table 6.5 shows that having $100 \%$ of the UE traces enables a highquality TAL design. However, the results from Table 6.6 show that 
Table 6.6: Signaling overheads of the TAL design obtained from Scheme 3.

\begin{tabular}{c|rcr|crr}
\hline & \multicolumn{3}{|c|}{ without cost } & \multicolumn{3}{c}{ cost added } \\
Scenario No. & TAU & Paging & Total & TAU & Paging & Total \\
\hline 1 & 267 & 611.3 & 878.3 & 656 & 611.3 & 1267.3 \\
2 & 282 & 585.0 & 867.0 & 637 & 585.0 & 1222.0 \\
3 & 276 & 624.7 & 900.7 & 635 & 624.7 & 1259.7 \\
4 & 321 & 521.9 & 842.9 & 686 & 521.9 & 1207.9 \\
5 & 294 & 642.4 & 936.4 & 662 & 642.4 & 1304.4 \\
6 & 386 & 340.2 & 726.2 & 737 & 340.2 & 1077.2 \\
7 & 385 & 469.0 & 854.0 & 740 & 469.0 & 1209.0 \\
8 & 635 & 375.9 & 1010.9 & 1018 & 375.9 & 1393.9 \\
9 & 315 & 504.8 & 819.8 & 670 & 504.8 & 1174.8 \\
10 & 468 & 427.7 & 895.7 & 855 & 427.7 & 1282.7 \\
\hline
\end{tabular}

for most of these UE-traces scenarios, an optimized TAL based on 5\% of UE traces is not a convincing design. A higher percentage of UEtraces must be considered in order to improve the performance of the scheme.

- Considering the very time-consuming process of designing TAL3 for even a small portion of the UEs, and the fact that TAL3 is not achieving significant improvement in the total signaling overhead, this scheme is not recommended.

- Comparing the amount of improvement in the total signaling overhead to the cost of collecting the UE traces, it is apparent that collecting the UE traces by TAL is not advantageous.

\subsection{Conclusions}

In this chapter, three TAL schemes were proposed, and their performances were evaluated, and compared to each other and to the optimal standard TA scheme in terms of baseline. The numerical results from this study showed that, generally, the TAL schemes can significantly reduce the signaling overhead compared to the standard TA scheme in a large-scale network.

The first scheme, which designs TAL independent from UE traces, performed best in large-scale networks. The "rule of thumb" is a very fast approach to obtaining a good TAL assignment. However, because it only con- 
siders the one-hop neighbors of the TAL of each cell, the design may not perform well for some scenarios, such as in the train scenario for which it is more reasonable to include more cells forming paths in the TAL. Another conclusion is that designing TAL based on UE traces results in a design which only performs well for the traces collected, and could not be generalized to a large-scale network. 



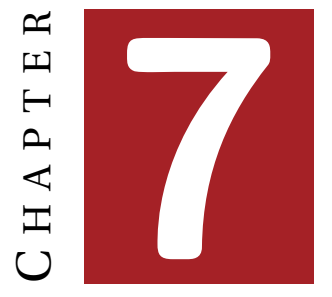

\section{Reducing Signaling Overhead}

Since the introduction of the TAL concept for LTE networks, defeating some of the existing limitations of the standard TA design has been expected. However, how to exploit this flexibility in the system, without the existence of UEs' mobility traces, was ambiguous. The TAL optimization formulation in this chapter allows a cell to have a collection of potentially overlapping TALs to be assigned to its UEs. Due to the objective function of reducing the total signaling overhead, the optimal solution is to assign one specific TAL to all UEs being updated in that cell. This takes us back to the principles of the TAL designs presented in Chapter 6. However, the proposed overlapping TAL design is able to explore the potential of the list concept to a wider extent compared to what has been done previously. Unlike the previous schemes in which solutions better than the standard TA scheme were heuristically found, in the current scheme the optimal TAL design in terms of the total signaling overhead is found for a snapshot in time.

The linear program (LP) optimization framework used in this chapter is based on the use of LTE TALs, which may contain overlapping TAs. To optimize the total signaling overhead of the overlapping TAL scheme, the same trade-off which previously existed in the standard TA scheme on the size of TAs, now exists for the length of each list in terms of the number of TAs they contain [78]. Frequent TAUs increase the load in MME and UE battery consumption. It also reduces the success rate of paging, as the UE is not able to respond to paging while it is performing a TAU [110]. On the other hand, long lists would increase the downlink signalings from the MME. Technically, up to 16 TAs per TAL are supported by LTE [5]. This constraint has been added to the formulation of the overlapping TAL scheme. 


\subsection{The Linear Programming Model}

A TA as small as a single cell is considered. This means that there will be no restriction on a given TA layout, and it helps to explore the flexibility of the overlapping TAL to its limit.

The proportional use of TAL $\ell$ for cell $i$ is denoted by a non-negative optimization variable $x_{i \ell}$. The binary indicator $a_{i \ell}$ indicates whether or not the cell $i$ is in TAL $\ell$. The minimization of the total overhead from TAU and paging can be modeled by the following LP model. The description of notations is also given in Appendix A.

$$
\begin{aligned}
& \min \sum_{i \in \mathscr{N}} \sum_{j \in \mathscr{N}: i \neq j} c_{T A U}(i, j)+\sum_{i \in \mathscr{N}} c_{\text {paging }}(i) \\
& \text { s.t. } \sum_{\ell \in \mathscr{L}} a_{i \ell} x_{i \ell}=1, \quad i \in \mathscr{N} \\
& c^{u}\left(h_{i j} \sum_{\ell \in \mathscr{L}} a_{i \ell}\left(1-a_{j \ell}\right) x_{i \ell}+h_{j i} \sum_{\ell \in \mathscr{L}} a_{j \ell}\left(1-a_{i \ell}\right) x_{i \ell}\right)=c_{T A U}(i, j), \\
& i, j \in \mathscr{N}, i \neq j \\
& \alpha c^{p} \sum_{\ell \in \mathscr{L}} \sum_{i \in \ell} \sum_{j \in \ell, i \neq j} u_{i} a_{j \ell} x_{i \ell}=c_{\text {paging }}(i), \\
& i \in \mathscr{N} \\
& \sum_{i \in \mathcal{N}} a_{i \ell} \leq N_{T A L} \\
& \ell \in \mathscr{L} \\
& x_{i \ell} \geq 0 \text {, } \\
& \ell \in \mathscr{L}, i \in \mathscr{N} .
\end{aligned}
$$

The first set of constraints (7.1b) assures that the proportional usage values of TALs in each cell $i$ add up to one. This means that all UEs in each cell will be assigned a TAL. The second set of constraints (7.1c) computes the TAU between each two different cells $i$ and $j$ in the network. For each ordered pair $i$ and $j$, if $i$ but not $j$ is included in TAL $\ell$ (i.e., $a_{i \ell}=1, a_{j \ell}=0$ ), then there is a TAU overhead of $c^{u} h_{i j} x_{i \ell}$ between them. The third set of constraints (7.1d) calculates the paging overhead of each cell $i$ in the network. For any list $\ell$ in cell $i$, the overhead for paging UEs in cell $j \in \ell$ is $\alpha c^{p} u_{i}$ scaled by the number of cells in $\ell$ and the variable $x_{i \ell}$ which stands for the proportional use of $\ell$. The fourth set of constraints (7.1e) assures that the length of TALs does not exceed the limit of $N_{T A L}$, which is the maximum allowable number of TAs in TAL. 


\subsection{Solution Characteristics}

The number of possible lists for each cell is $2^{N-1}$, therefore the number of variables of the above LP model is exponential in the number of cells $N$. However, due to the LP formulation, it can be solved efficiently even for a large number of variables. The following proposition provides a solution characterization.

PROPOSITION 3. At the optimum solution of (7.1), for any cell $i \in \mathscr{N}$, there is exactly one $\ell \in \mathscr{L}$, such that in $x_{i \ell}=1$, and the rest are zeros.

PROOF. Suppose $\ell_{1} \in \mathscr{L}$ and $\ell_{2} \in \mathscr{L}\left(\ell_{1} \neq \ell_{2}\right)$ are both present at the optimum solution for cell $i$, meaning that $a_{i \ell_{1}}=a_{i \ell_{2}}=1, x_{i \ell_{1}}+x_{i \ell_{2}}=1, x_{i \ell_{1}}>0$ and $x_{i \ell_{2}}>0$. Then the objective function value for cell $i$ is:

$$
\sum_{j \in \mathscr{N}: i \neq j} c^{u} h_{i j}\left(\left(1-a_{j \ell_{1}}\right) x_{i \ell_{1}}+\left(1-a_{j \ell_{2}}\right) x_{i \ell_{2}}\right)+\alpha c^{p} u_{i}\left(a_{j \ell_{1}} x_{i \ell_{1}}+a_{j \ell_{2}} x_{i \ell_{2}}\right)
$$

Because $\ell_{1}$ and $\ell_{2}$ are different, there must be at least one cell $j^{\prime}$ that exists in one list and does not exist in the other list, say $a_{j^{\prime} \ell_{1}}=1$, and $a_{j^{\prime} \ell_{2}}=$ 0 . With this observation, the corresponding term in the above sum for $j^{\prime}$ becomes:

$$
c^{u} h_{i j^{\prime}} x_{i \ell_{2}}+\alpha c^{p} u_{i} x_{i \ell_{1}}
$$

Among the two values $c^{u} h_{i j^{\prime}}$ and $\alpha c^{p} u_{i}$, one has a lower value. According to the objective function which is to minimize the total signaling overhead, if $c^{u} h_{i j^{\prime}}$ is lower, then setting $x_{i \ell_{1}}=0$ and $x_{i \ell_{2}}=1$ results in a better objective value, and if $\alpha c^{p} u_{i}$ is lower, then setting $x_{i \ell_{1}}=1$ and $x_{i \ell_{2}}=0$ gives a better objective value. However, this is in contradiction to the earlier assumption that both $x_{i \ell_{1}}$ and $x_{i \ell_{2}}$ are greater than zero at optimum. This shows that for any cell $i$, an extreme solution is optimum for the trade-off between update and paging overheads while considering each cell $j^{\prime}$ to be included in the list of $i$ or not. The same logic applies in considering more than two lists in the optimum solution. Hence the conclusion.

Here, it can be concluded that while using the overlapping TAL scheme for minimizing the total signaling overhead, each cell assigns one specific TAL to all UEs being registered in that cell. What is stated in the proposition is in fact very interesting. Although each cell in the overlapping TAL scheme can use different proportions of TALs, meaning that $x_{i \ell}$ in the formulation 
can be any non-negative value, due to the objective function in (7.1a), for each cell there is only one TAL which results in the minimum value of the total signaling overhead. This fact sets a proper fundamental for the TAL schemes presented in the previous chapter. The solution to the proposed LP model is a lower bound to the performance of the "rule of thumb".

For a snapshot scenario (i.e. given $u_{i}$ and $h_{i j}$ for all $i, j \in \mathscr{N}$ ), the optimum overlapping TAL design solution of the proposed LP model is a lower bound to the total signaling overhead of any TAL-to-cell assignment for the aggregated data of UEs. This is equivalent to finding the optimum binary $\mathbf{S}(\mathbf{t})$ matrix, in which the symmetric and transitive properties are relaxed. The total signaling overhead of this binary matrix is lower than any fractional $\mathbf{S}(\mathbf{t})$ matrix. Clearly in the optimum TAL design, for any $i, j \in \mathscr{N}$ and $i \neq j$, the lower value between $c^{u} h_{i j}$ and $\alpha c^{p} u_{i}$ is chosen. Here, it can be concluded that the optimum overlapping TAL configuration from the LP model performs optimally for a limited period of time while considering all UEs have been updated to the TALs of the cells they are located in.

As the UEs are allowed to move freely among the cells included in their TALs, eventually each cell will contain UEs with different TALs. This challenge has been previously explained in Section 5.3. In the current study the total signaling overhead of the optimum TAL design from the LP model may in reality be far from the one computed in (7.1a), as the scheme is vulnerable to the UE traces inside the network. Hence, for performance evaluation of the TAL solution, this vulnerability should be considered.

\subsection{Performance Evaluation}

To evaluate the performance of the proposed overlapping TAL scheme on a large-scale topology, the Lisbon network is again considered. As splitting the cells of a site into different TAs is not a common practice, the LP model is applied at site level.

As the LP model is exponential in the number of sites, applying it to the entire network is a time-consuming process. One solution is to divide the network to smaller sets of sites and apply the model to each set separately. According to constraint (7.1e), the model itself will divide the network into sets of maximum $N_{T A L}$. The actual value by $3 \mathrm{GPP}$ standard $N_{T A L}=16$ is set here. To ensure the optimum division of sites in the network, the integer programming model in [109] is used while adding the constraint defined in (7.4). The fact that the site is always included in the lists given by itself is also considered in the model. 


$$
\sum_{j \in \mathscr{N}} s_{i j}(\mathbf{t}) \leq 15, \quad i \in \mathscr{N}, i \neq j
$$

The model is solved by CPLEX [60] for a very high value of $c^{u}$ (i.e. $c^{u} \gg$ $c^{p}$ ), which gives the optimum division of the network based on the total signaling overhead. After this procedure, constraint (7.1e) will become redundant. This grouping certainly restricts the performance of the overlapping TAL scheme, and the aggregated results from the model for the groups are no longer the lower bounds to the performance of the overlapping TAL. It should be noted that the model originally proposed is general and does not contain any grouping.

Figure 7.1 illustrates the Lisbon network topology and key statistics, and the underlying division of sites. In this performance evaluation, the overhead of a single TAU, $c^{u}$, is set at ten times the overhead of a single paging, $c^{p}$. The call intensity factor is set to $\alpha=0.05$. The standard TA design is computed using the model in [109] and CPLEX. Table 7.1 presents the numerical assumptions and values for this performance evaluation.

Table 7.1: Numerical assumptions and values for performance evaluation.

\begin{tabular}{lll}
\hline Parameter & Description & Values \\
$\alpha$ & Call intensity factor & 0.05 \\
$\gamma$ & Probability of UEs entering a site without TAU & {$[0,1]$} \\
$\delta$ & Proportion of UEs in a site assigned to TAL of other sites & {$[0,1]$} \\
$c^{p}$ & Cost of a single paging & 0.1 \\
$c^{u}$ & Cost of a single TAU & 1 \\
$N$ & Number of sites & 60 \\
$N_{T A L}$ & Maximum number of TAs in TAL & 16 \\
$T$ & Number of TAs & 7 \\
\hline
\end{tabular}

Figure 7.2 illustrates the optimum standard TA design for the network. TABLE 7.2 gives the TAU, paging and the total signaling overhead of the standard TA design and the initial site grouping shown in Figure 7.1. Clearly, the standard TA design should have the lowest total signaling overhead compared to any other group layout in the network. As the overlapping TAL scheme is applied inside each group presented in Figure 7.1, the TAU overhead of the design, $2.0683 \times 10^{4}$, should finally be added to the overhead resulting from the overlapping TAL scheme. This can be interpreted as the cost for making the groupings, since if there was not any grouping, the over- 


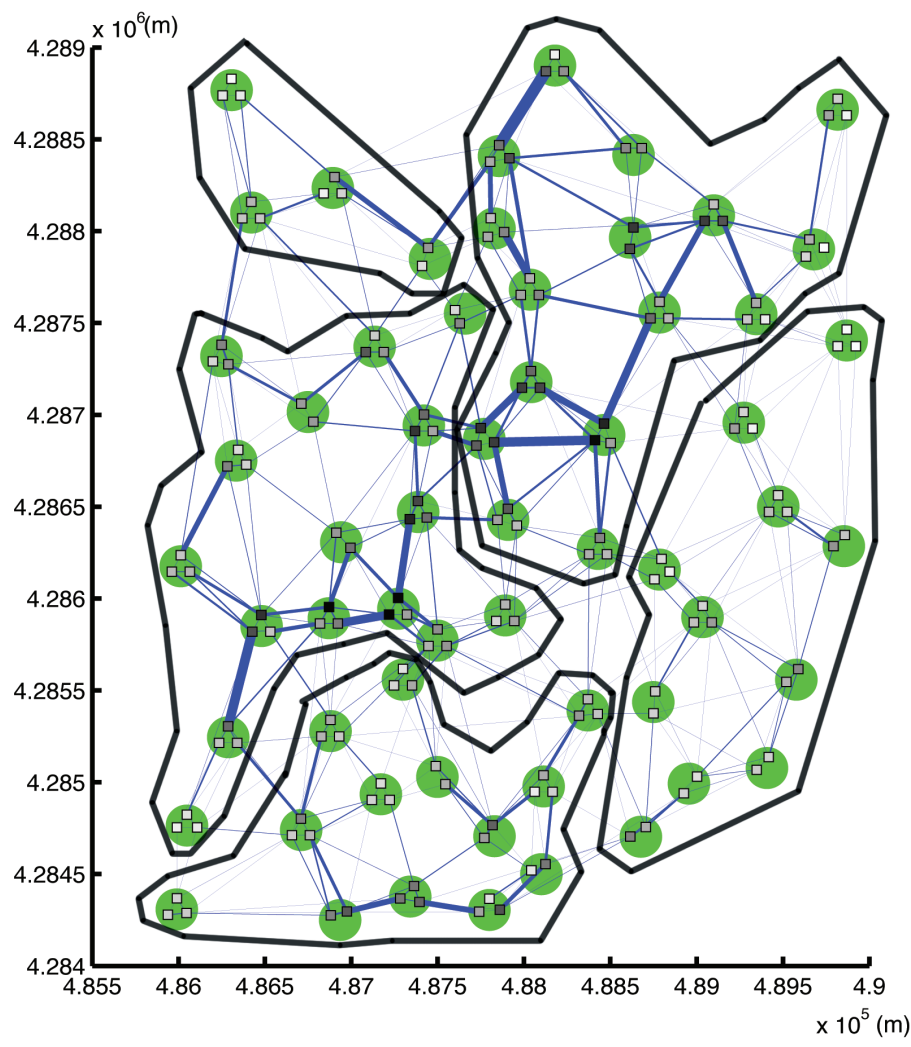

Figure 7.1: An illustration of the site groupings for the overlapping TAL scheme.

Table 7.2: Signaling overhead comparisons of Figures 7.1 and 7.2. Figure 7.2 represents the standard TA design.

\begin{tabular}{lccc}
\hline & TAU & Paging & Total \\
\hline Design in Figure 7.1 & $2.0683 \times 10^{4}$ & $2.3684 \times 10^{4}$ & $4.4367 \times 10^{4}$ \\
Design in Figure 7.2 & $2.3849 \times 10^{4}$ & $1.8603 \times 10^{4}$ & $4.2452 \times 10^{4}$ \\
\hline
\end{tabular}

lapping TAL model would have had more flexibility in choosing the sites included in each TAL.

To configure the overlapping TALs for the Lisbon network, the model in Section 7.1 is applied to each group in Figure 7.1. TABLE 7.3 presents the 


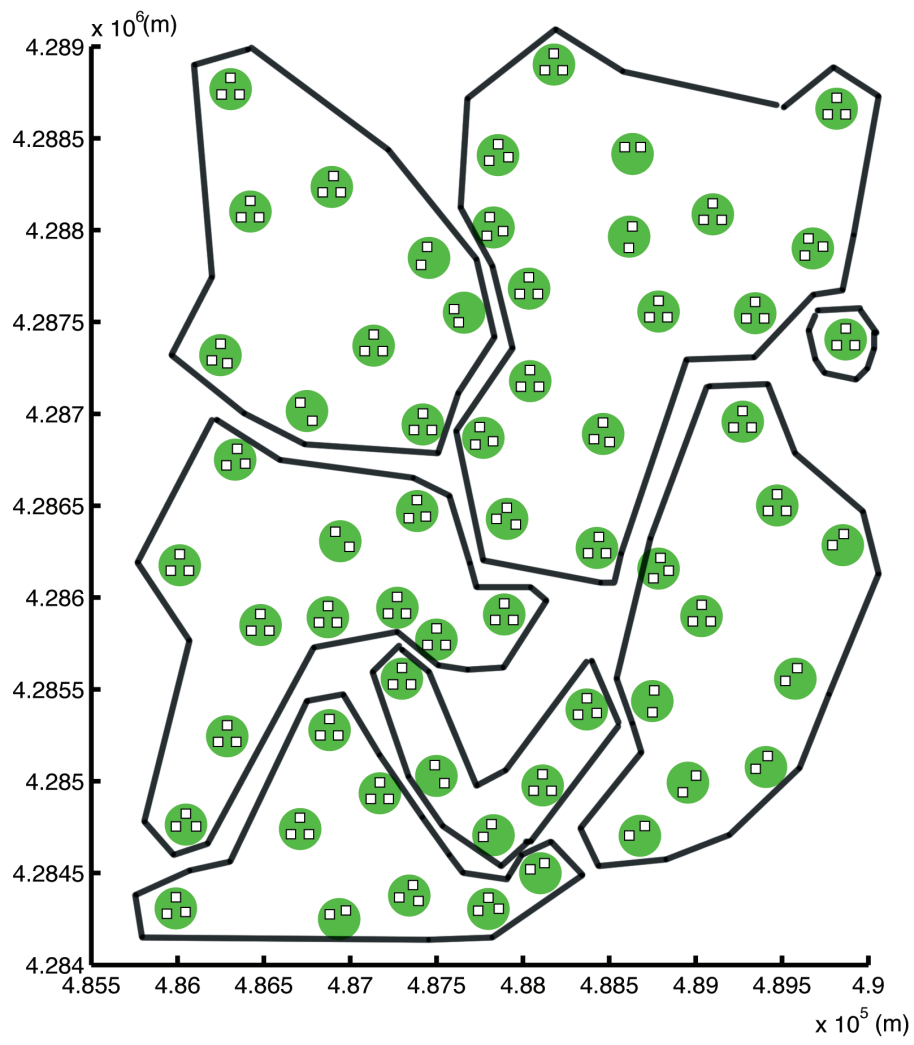

Figure 7.2: An illustration of the optimum standard TA design.

number of sites, the computed TAU, paging and the total signaling overhead for the overlapping TAL scheme for each group. The last column of the table presents the model computing time for each group. The computations are run on Intel Core 2 Duo of $2.1 \mathrm{GHz}$. One immediate observation is that the paging overhead accounts for most of the total signaling overhead of each group. The reason is that the network is already divided into groups of geographically close sites, most of which have a high amount of handover between them, and hence the model is intended to consider those sites in each others' TALs.

To give an insight into the design of overlapping TAL designs, TABLE 7.4 presents the load and handover of sites in Group 1, which contains four 
Table 7.3: The LP model results and computation time for each group

\begin{tabular}{ccrrrr}
\hline Group & No. of sites & TAU & Paging & Total & Time (s) \\
\hline $\mathbf{1}$ & 4 & 15 & 122.085 & 137.085 & 0.008 \\
$\mathbf{2}$ & 11 & 83 & 675.920 & 758.920 & 0.036 \\
$\mathbf{3}$ & 13 & 70 & 1114.230 & 1184.230 & 0.164 \\
$\mathbf{4}$ & 16 & 191 & 2485.755 & 2676.755 & 1.872 \\
$\mathbf{5}$ & 16 & 49 & 2548.445 & 2597.445 & 1.744 \\
\hline
\end{tabular}

Table 7.4: Load and handover data of Group 1 with their optimum TAL

\begin{tabular}{ccrrrrl}
\hline Site & Site Load & HO to 1 & HO to 2 & HO to 3 & HO to 4 & TAL \\
\hline $\mathbf{1}$ & 4057 & 0 & 542 & 15 & 483 & $\{1,2,4\}$ \\
$\mathbf{2}$ & 3570 & 201 & 0 & 1271 & 287 & $\{1,2,3,4\}$ \\
$\mathbf{3}$ & 2985 & 0 & 554 & 0 & 0 & $\{2,3\}$ \\
$\mathbf{4}$ & 1304 & 331 & 118 & 0 & 0 & $\{1,2,4\}$ \\
\hline
\end{tabular}

sites, and the optimum overlapping TALs for each site. This small example shows that:

- the LP model assigns each site with one specific TAL, which results to the minimum sum of TAU and paging overhead.

- sites in the group contain overlapping TALs.

- the symmetric and transitive property of $\mathbf{S}(\mathbf{t})$ matrix is relaxed, and hence the total signaling overhead is significantly reduced compared to the standard TA scheme. The total signaling overhead of the standard TA design for Group 1 is 178.74 computed by using the model in [109].

\subsubsection{Lower Bound to the Restricted Design}

By considering a snapshot of time in which all UEs in one site have updated their overlapping TALs from the sites they are located in, TABLE 7.5 presents the lower bound to the performance of the overlapping TAL scheme which is restricted by groupings. It is possible to compute this lower bound by summing up the values of each column in TABLE 7.3. The TAU overhead of Figure 7.1 should also be added to the TAU overhead values in TABLE 7.3. 
Table 7.5: Performance comparison of the two schemes in an ideal case.

\section{Overhead Standard TA Designed overlapping TAL Improvement}

\begin{tabular}{llcl} 
& & (lower bound) \\
\hline TAU & $2.3849 \times 10^{4}$ & $2.1091 \times 10^{4}$ & $11.6 \%$ \\
Paging & $1.8603 \times 10^{4}$ & $6.9464 \times 10^{3}$ & $62.7 \%$ \\
Total & $4.2452 \times 10^{4}$ & $2.8037 \times 10^{4}$ & $34.0 \%$ \\
\hline
\end{tabular}

TABLE 7.5 compares the overhead values of the standard TA scheme with the ones obtained by the overlapping TAL design for an "ideal" scenario.

The comparisons show that, ideally, the overlapping TAL design restricted by groupings is able to significantly (i.e. $34 \%$ ) improve the performance of the signaling overhead in terms of both TAU and paging costs. The reason for having 5.4 times more improvement in the paging overhead compared to the TAU overhead is the elimination of extra paging overhead by relaxing the symmetric and transitive property of the $\mathbf{S}(\mathbf{t})$ matrix.

\subsubsection{Empirical Validation of the Design}

To justify the effectiveness of the proposed overlapping TALs, the performance of the scheme is analyzed based on different realistic UE movement scenarios. One advantage of the proposed scheme is the availability of the exact possible TALs that the UEs in one site may have. Let us consider site 2 of the small group in TABLE 7.4. As all other sites in the group have $\{2\}$ in their TALs, the UEs moving from any of the sites in the group to this site do not perform any TAU. It is obvious that, as time passes, the number of UEs having the TAL $\{1,2,3,4\}$ decreases in site 2 , as no UE from any of the sites in the group will update to the TAL of site 2 . On the other hand, UEs with the list of site 2 will not perform any update while moving to any other sites in the group.

Two methods are used for calculating the signaling overhead of the overlapping TAL design while considering different UEs' distribution. Both methods use the aggregated data of UEs, and not any specific UE-trace scenario. Both methods are expected to generate close results, as they, to some extent, share the same fundamentals. 


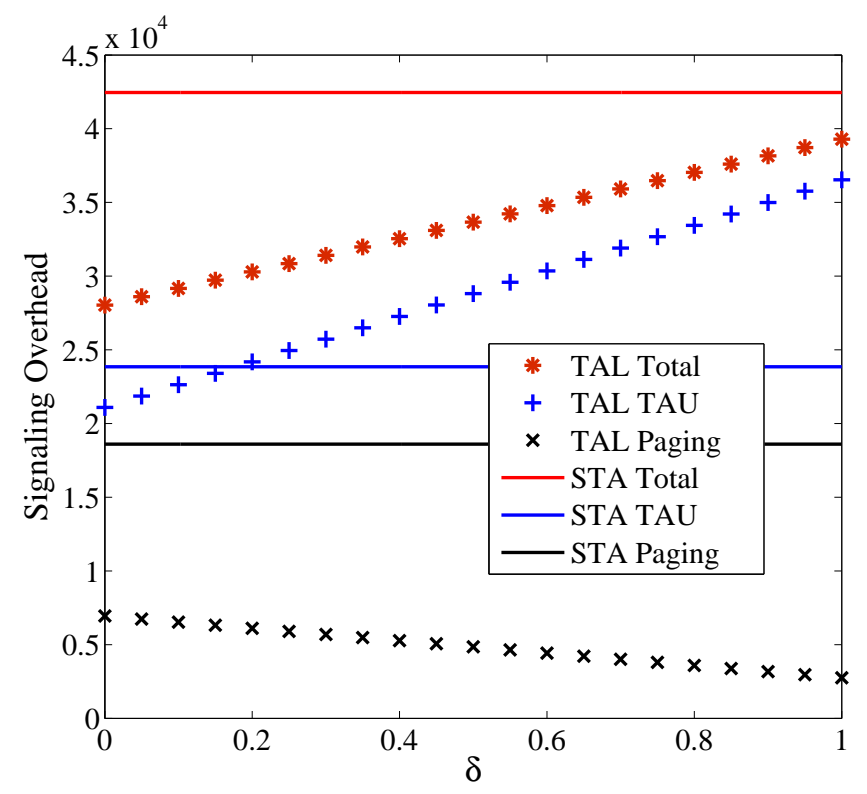

Figure 7.3: Evaluating the overlapping TAL scheme by Method I.

\subsubsection{Method I}

Factor $\delta$ represents the proportion of UEs in site $i$ having a TAL other than the one assigned by $i$. Hence, $\delta=0$ corresponds to the overheads presented in TABLE 7.5 for the overlapping TAL design. By increasing the value of $\delta$, the number of UEs with other TALs inside $i$ is increased. The population of UEs having TAL of site $j$ in $i$ is naturally assumed to be a portion of the handover from $j$ to $i$. Figure 7.3 compares the signaling overheads resulting from Method I for different values of $\delta \in[0,1]$ with the standard TA design. The results show that the proposed overlapping TAL scheme is promising in terms of reducing the total signaling overhead under different UE-distribution situations.

\subsubsection{Method II}

The definition of $s_{i j}$ given in (6.1) and presented in Section 6.1 is re-used here. The parameter $l_{i j}$, which is defined 1 if $j$ is in the TAL of $i$ and 0 otherwise, can be obtained from $x_{i \ell}$ solutions of the overlapping TAL design. Figure 7.4 compares the signaling overheads resulting from Method II for 


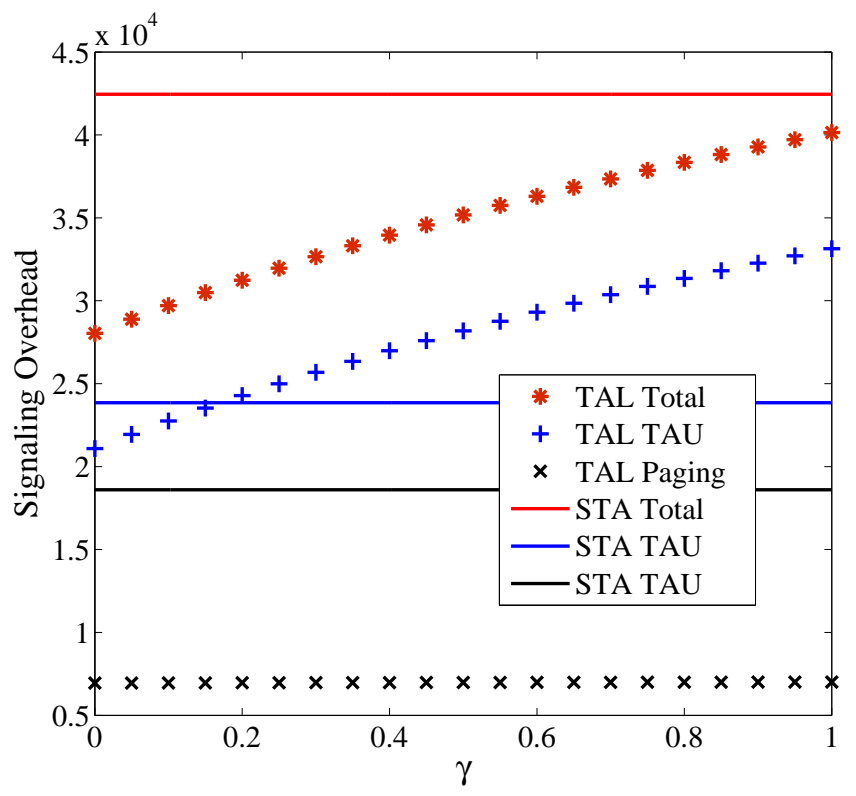

Figure 7.4: Evaluating the overlapping TAL scheme by Method II.

all values of $\gamma$ with the standard TA scheme. As before, the results show that the proposed scheme is able to improve the performance of the TALheterogeneous network.

Figure 7.5 shows that the results from the two methods are very close to each other, as they both estimate the UE's distribution based on the load and handover of the network.

\subsection{Conclusions}

In this chapter, a linear programming model was proposed to configure overlapping tracking area lists (TALs) for the purpose of minimizing the total signaling overhead of location management in LTE networks. An optimum overlapping TAL design was allocated to each cell in order to minimize the total signaling overhead. It has been proved that the optimum overlapping TAL solution for this problem is that each cell assigns one specific TAL to all UEs being registered in that cell. The LTE constraint on the number of TAs in a list was considered and formulated in the model. Two separate methods were used to evaluate the performance of the overlapping TALs with- 


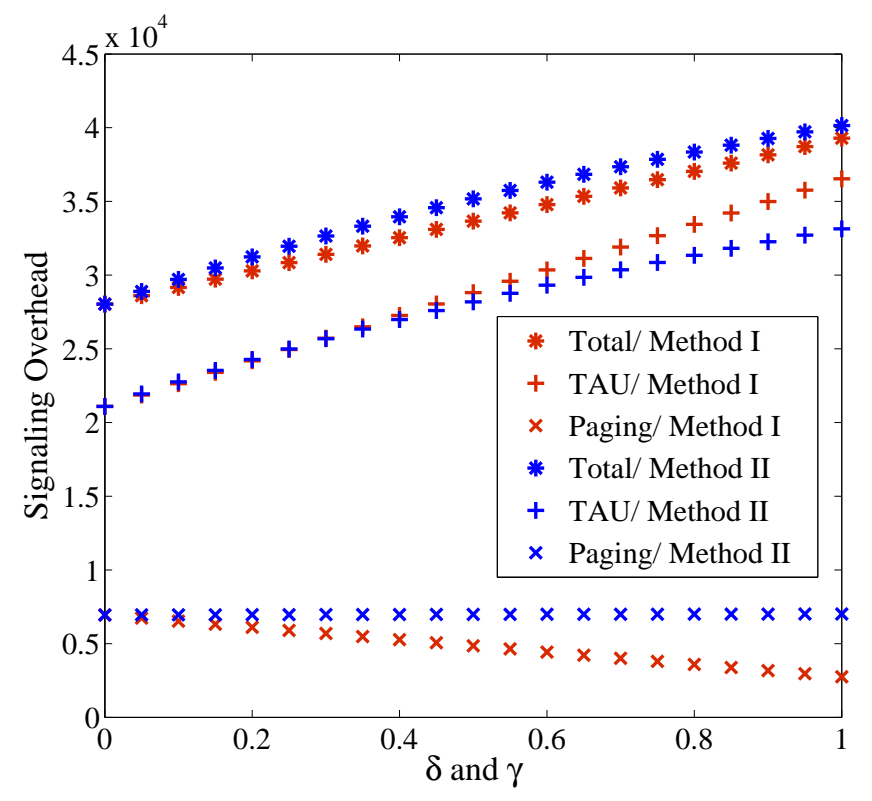

Figure 7.5: Comparison of the overlapping TAL overheads by Method I and II.

out considering any specific UE-trace scenario. The results showed that the optimized TALs outperform the standard TA scheme in reducing the total signaling overhead. 


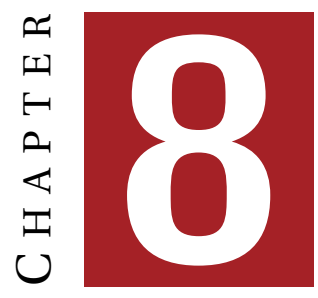

\section{Signaling Congestion Mitigation of THE TRAin SCENARIO}

The signaling congestion problem of the train scenario has been one of the most challenging problems of the standard TA design. The problem has previously been explained in Chapter 5 . In this chapter, a modified overlapping TAL scheme is developed to mitigate the signaling congestion due to simultaneous mass mobility. An LP model which minimizes the number of TAUs while keeping the total paging overhead lower than some pre-defined limit, is presented.

The congestion mitigation problem of this chapter has also been considered in [74]. The authors of there used a randomized time distribution for the initiation of the process of cell re-selections to avoid the mass mobility signaling congestion. However, their mechanism requires additional configuration on the network side to provide the algorithm parameters, while the approach presented here uses only existing features of the standard LTE systems.

One of the main challenges in applying TAL is the estimation of the total signaling overhead of a fully deployed TAL scheme in a large-scale network. In the modified overlapping TAL scheme presented in this chapter, the signaling overhead calculation is independent of UE traces. The numerical results demonstrate that the proposed model is able to balance the load in some representative scenarios of massive mobility signaling congestion. The study in this chapter has been appeared in [84]. 


\subsection{Modified Overlapping Tracking Area List}

In order to mitigate the TAU signaling congestion, some extra paging overhead is introduced, and to mitigate the paging signaling congestion, addition of some extra TAU overhead is inevitable. Therefore, towards avoiding signaling congestion, the signaling overhead calculation of TAU and paging should be considered, separately. Hence, Equation (2.2) is divided into Equations (8.1) and (8.2) for TAU and paging signaling overhead, respectively.

$$
\begin{gathered}
C^{U}=\sum_{i \in \mathcal{N}} \sum_{j \in \mathcal{N}: i \neq j} c_{T A U}(i, j)=\sum_{i \in \mathcal{N}} \sum_{j \in \mathscr{N}: j \neq i} c^{u} h_{i j}\left(1-s_{i j}(\mathbf{t})\right) \\
C^{P}=\sum_{i \in \mathscr{N}} c_{\text {paging }}(i)=\sum_{i \in \mathcal{N}} \sum_{j \in \mathscr{N}: j \neq i} \alpha c^{p} u_{i} s_{i j}(\mathbf{t})
\end{gathered}
$$

In Equations (8.1) and (8.2), $C^{U}$ denotes the total TAU signaling overhead, and $C^{P}$ denotes the total paging signaling overhead for a given TA design $\mathbf{S}(\mathbf{t})$. In both equations, the middle equality terms relate the parameters to the ones defined in Chapter 7.

In the modified overlapping TAL scheme, each cell uses multiple and overlapping TALs to be allocated to UEs updating their TALs from the cell. The following subsection explains the modified overlapping TAL scheme with a network of three cells.

\subsubsection{An Instructive Example}

Figure 8.1 considers the three cells, A, B, and C, along a train path. The optimum standard TA configuration of this part of the network is one of the four possible combinations presented in Table 8.1. The parentheses in the second column of the table specify different groupings of these three cells. The configuration $\{(A, C),(B)\}$ is not a feasible combination, as by construction of this example there is no direct handover from A to C. According to Equations (8.1) and (8.2), the total TAU and paging overheads are calculated for each of the four configurations.

If the transitivity condition of the $\mathbf{S}(\mathbf{t})$ matrix is relaxed, then $\mathbf{S}_{\text {new }}(\mathbf{t})$ matrix in (8.3) could become a possible configuration for the example in Figure 8.1. 


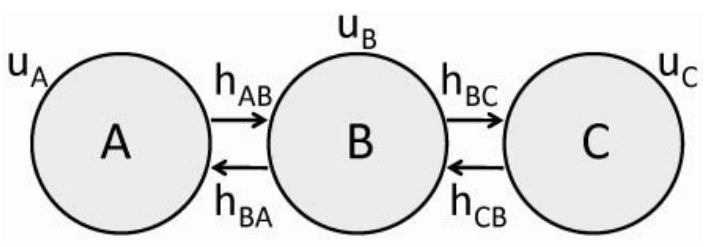

Figure 8.1: Three cells along a train path.

Table 8.1: Possible TA configurations, $C^{U}$ and $C^{P}$ of the ABC network.

\begin{tabular}{llll}
\hline No. & Configuration & $C^{U}$ & $C^{P}$ \\
\hline 1 & $(\mathrm{~A}),(\mathrm{B}),(\mathrm{C})$ & $c^{u}\left(h_{A B}+h_{B A}+h_{B C}+h_{C B}\right)$ & 0 \\
\hline 2 & $(\mathrm{~A}, \mathrm{~B}),(\mathrm{C})$ & $c^{u}\left(h_{B C}+h_{C B}\right)$ & $\alpha c^{p}\left(u_{A}+u_{B}\right)$ \\
\hline 3 & $(\mathrm{~A}),(\mathrm{B}, \mathrm{C})$ & $c^{u}\left(h_{A B}+h_{B A}\right)$ & $\alpha c^{p}\left(u_{B}+u_{C}\right)$ \\
\hline 4 & $(\mathrm{~A}, \mathrm{~B}, \mathrm{C})$ & 0 & $2 \alpha c^{p}\left(u_{A}+u_{B}+u_{C}\right)$ \\
\hline
\end{tabular}

$$
\begin{gathered}
\mathbf{S}_{\text {new }}(\mathbf{t})=\left(\begin{array}{lll}
1 & 1 & 0 \\
1 & 1 & 1 \\
0 & 1 & 1
\end{array}\right) \\
C_{n e w}^{U}+C_{n e w}^{P}=c^{u}\left(h_{A C}+h_{C A}\right)+\alpha c^{p}\left(u_{A}+2 u_{B}+u_{C}\right)
\end{gathered}
$$

The total signaling overhead of $\mathbf{S}_{n e w}(\mathbf{t})$ is given in (8.4). In the example, $h_{A C}=h_{C A}=0$, and $C_{n e w}^{U}=0$, and hence the $\mathbf{S}_{\text {new }}(\mathbf{t})$ configuration has the lowest TAU overhead compared to the configurations in Table 8.1. Assume that $u_{A} \gg u_{B}$ and $u_{C} \gg u_{B}$ (for example considering that there are train stations in $A$ and $C$ with large number of UEs standing in the platform), then the paging overhead $C_{n e w}^{P}$ is almost half of the paging overhead of the fourth configuration in the table.

This example shows that the transitive property of the $\mathbf{S}(\mathbf{t})$ matrix can limit the performance of the standard TA scheme. Here, it is of interest to show that the TAL concept has the potential to reduce the overhead by relaxing this property, and achieving an effect similar to that of $\mathbf{S}_{\text {new }}(\mathbf{t})$. However, the challenge in using the list concept is that the UEs in one cell might have different lists depending on the cells from which the lists are assigned. One can refer back to the discussion in Section 5.3.

By assuming that each of the cells A, B and C are a separate TA (configuration 1) and they can assign UEs with different TALs, Table 8.2 presents the 
Table 8.2: The list of TALs for each cell in the ABC network of Figure 8.1.

\begin{tabular}{lll}
\hline $\mathrm{A}$ & $\mathrm{B}$ & $\mathrm{C}$ \\
\hline$\{A\}$ & $\{B\}$ & $\{C\}$ \\
$\{A, B\}$ & $\{A, B\}$ & $\{B, C\}$ \\
$\{A, B, C\}$ & $\{B, C\}$ & $\{A, B, C\}$ \\
& $\{A, B, C\}$ &
\end{tabular}

set of all possible TALs for each cell. The TALs assigned by each cell should contain that cell. Cells A and $\mathrm{C}$ are not giving TAL $\{A, C\}$ to their UEs as there is no direct handover between these two cells; this list only increase the paging overhead without any improvement in TAU. The table shows that the UEs in the ABC network have the possibility to be assigned one of the following six TALs: $(\{A\},\{B\},\{C\},\{A, B\},\{B, C\},\{A, B, C\})$.

Let $t a$ denote any TA, and $t a l$ denote any TAL of the ABC network; $X_{t a l}^{t a}$ is defined as the percentage of the UEs inside each $t a$ having tal as their list. Consider the following three equations. Equation (8.5) assures that all the UEs receiving their lists from $A$ use one of the three candidate TALs of $A$. Equations (8.6) and (8.7) express the same logic for TAs B and C, respectively.

$$
\begin{array}{r}
X_{A}^{A}+X_{A B}^{A}+X_{A B C}^{A}=1 \\
X_{B}^{B}+X_{A B}^{B}+X_{B C}^{B}+X_{A B C}^{B}=1 \\
X_{C}^{C}+X_{B C}^{C}+X_{A B C}^{C}=1
\end{array}
$$

By the definition of TAL, the UEs assigned to the list $\{A, B, C\}$ can move in the $\mathrm{ABC}$ network without any TAU, and they could have also been updated to this TAL from any of the cells/TAs. To make the calculation of signaling overhead tractable, the restriction that the percentage of assigning a common TAL from different TAs are equal is imposed. This means that $X_{A B}^{A}=X_{A B}^{B}, X_{B C}^{B}=X_{B C}^{C}$ and $X_{A B C}^{A}=X_{A B C}^{B}=X_{A B C}^{C}$. Thus, there is no need to continue with the superscripts of elements $\left(X_{\text {tal }}\right)$.

In the overlapping TAL scheme, the cells inside each TA are assigned a list of overlapping TALs. The cells update the UEs with different TALs in their lists. The standard TA design is considered as the backbone structure, and the lists are created by different combinations of TAs. In the modified overlapping TAL scheme, the restriction explained in the example is applied. This means that in the modified overlapping TAL scheme, the percentages 
of assigning a common list from different cells to the UEs in the network are equal.

The biggest difficulty in calculating the signaling overhead was having UEs with different TALs in one cell. With the assumption on the definition of $X_{t a l}$ and the modified overlapping TAL scheme, the percentage of UEs assigned to each TAL is known. Therefore, unlike previous studies where the signaling overhead was calculated by following each UE movement pattern and call arrivals or estimated by methods such as the ones presented in Sections 7.3.2.1 and 7.3.2.2, here the TAU and paging overhead formulas given in (8.1) and (8.2) can be directly used.

From now on and throughout the rest of the dissertation the term overlapping TAL scheme refers to this modified scheme, and for simplicity the word modified is omitted.

\subsection{An LP Model for the Train Scenario}

To overcome the signaling congestion in the train scenario, the cells along the railway path can assign different TALs to the UEs inside the train, this was previously shown in Figure 5.4. As the TAs involved in the train scenario form a line such as the one in Figure 8.2, the LP formulation has a simple structure. UE movement occurs only between consecutive TAs along the line. Thus, the TALs contain blocks of consecutive cells, and the formulation's size becomes polynomial in the number of TAs. As numbering the TAs follows naturally by their positions along the line, for convenience, the position indices are used to refer to TAs. Hence, TA $i, i=1, . ., T$, is the $i$ th TA on the line. Each TAL is uniquely identified by its starting and ending TAs, therefore the variable definition is simplified to $x_{i j}$ for the TAL which contains all TAs from $i$ to $j$.

A min-max approach for TAU congestion mitigation is applied, that is, TALs are used to minimize the maximum TAU between all pairs of TAs. To this end, an upper bound on the increase of the total paging overhead is necessary, as otherwise the optimum would be to completely eliminate TAU by exclusively using the TAL containing all TAs (i.e., as if all cells form one TA). The bound is expressed as $\beta C^{P}$, where $C^{P}$ is the total paging overhead of TA design $\mathscr{T}$, and $\beta$ is a non-negative parameter denoting the allowed percentage of increase. An auxiliary variable $z$ is used to denote the maximum TAU between TAs. The min-max TAU optimization problem for the train scenario can then be modeled by the following LP. 


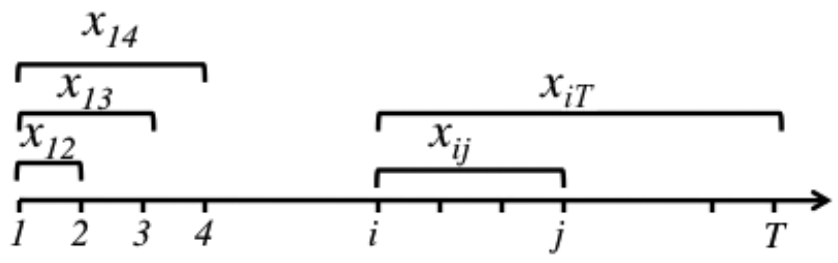

Figure 8.2: Definition of variables for the train scenario.

$\min z$

$$
\begin{array}{ll}
\text { s.t. } & \sum_{i=1}^{k} \sum_{j=k}^{T} x_{i j}=1, \\
& c^{u}\left(h_{i, i+1} \sum_{k=1}^{i} x_{k i}+h_{i+1, i} \sum_{k=i+1}^{T} x_{i+1, k}\right) \leq z, \\
& \alpha c^{p} \sum_{i=1}^{T-1} \sum_{j=i+1}^{T} \sum_{k=i}^{j} \sum_{h=k+1}^{j}\left(u_{k} n_{h}+u_{h} n_{k}\right) x_{i j} \leq \beta C^{P}, \\
& x_{i j} \geq 0,
\end{array}
$$

The first set of constraints (8.8b) ensures that each TA assigns all its UEs with feasible TALs. For the train scenario, it is sufficient to account for UE movements between adjacent TAs on the line, as can be seen in (8.8c). In (8.8d), paging is considered for all TA pairs of a line segment, and further over all the segments. Apart from the non-negativity requirement, the numbers of variables and constraints in (8.8) are of $O\left(T^{2}\right)$ and $O(T)$, respectively. Thus, the optimization approach of the train scenario has low complexity.

While considering two consecutive TAs, $i$ and $i+1$, it can be easily verified that there are a total of $i(T-i+1)$ TALs containing $i$, and, among them, $i$ TALs do not contain $i+1$. Thus, the amount of TAU generated by UE movement from $i$ to $i+1$ depends on the proportional usage levels of the $i$ TALs. The values are strictly between zero and one at optimum, thus not all of the UEs inside the train will perform TAU at a TA boundary, giving the desired effect of TAU congestion mitigation. 


\subsection{Performance Evaluation}

To evaluate the performance of the overlapping TAL scheme, two realistic congestion scenarios are constructed for a defined baseline scenario. Both congestion scenarios represent UE movement by train. An assumption is that all passengers inside the train hold cell phones. The aim is to illustrate that the overlapping TAL scheme can mitigate the congestion problem in both scenarios of the network. As the LP model is polynomial in size, applying it to large-scale scenarios of this kind is scalable. The basic element of this study is TA rather than cell. To compare the performance of the overlapping TAL scheme with the standard TA scheme, the TALs are considered to be the combinations of overlapping TAs. However, it is obvious that the TALs at cell level can improve the possible potentials of TALs.

The LP model defined in (8.8) has been implemented in the CPLEX optimizer [60]. The load and handover statistics of the UEs are given for one hour period of time. Table 8.3 gives the numerical assumptions and values for this performance evaluation.

Table 8.3: Numerical assumptions and values for performance evaluation.

\begin{tabular}{lll}
\hline Parameter & Description & Values \\
$\alpha$ & Call intensity factor & 0.05 \\
$\beta$ & Allowed percentage increase on the total paging & {$[0,0.8]$} \\
$c^{p}$ & Cost of a single paging & 0.1 \\
$c^{u}$ & Cost of a single TAU & 1 \\
$C^{P}$ & Total paging overhead of the baseline scenario & 719.7 \\
$C^{U}$ & Total TAU overhead of the baseline scenario & 122 \\
$N$ & Number of cells & 25 \\
$T$ & Number of TAs & 5 \\
\hline
\end{tabular}

\subsubsection{Baseline Scenario}

A railway path with five TAs $\{A, B, C, D, E\}$ and length of $150 \mathrm{~km}$ is defined as a baseline scenario. Each TA covers a large area and contains five cells. The boundaries of each TA have the lowest handovers between UEs in that region. Suppose that this configuration is the optimum standard TA design by model [109] based on the total signaling overhead of this specified part of the network. The total signaling overhead is $841.7\left(C^{U}=122, C^{P}=719.7\right)$. The data excludes the mass mobility of UEs in train. 


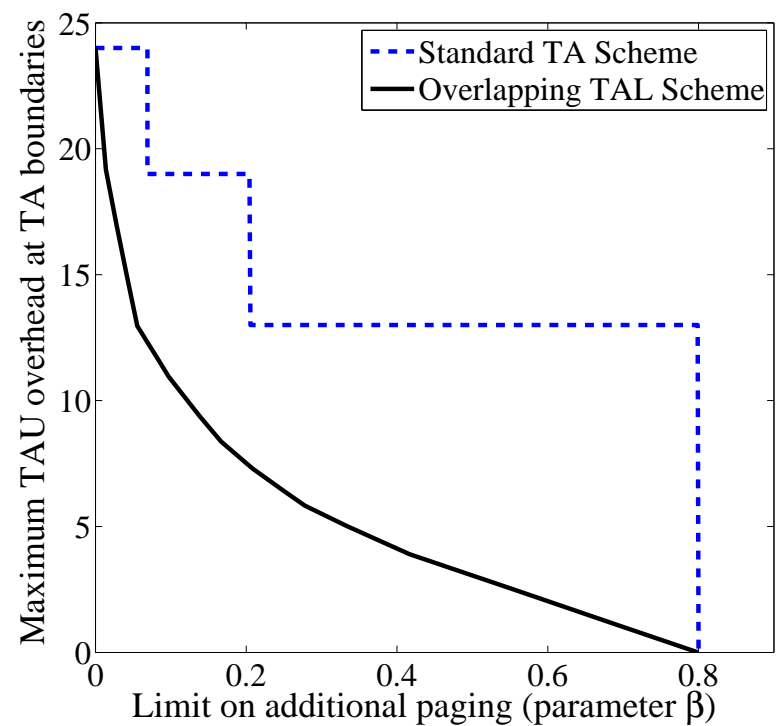

Figure 8.3: Evaluating the two schemes for the baseline scenario.

The model (8.8) is applied to the baseline scenario and is run for all $\beta$ values from 0 to maximum. It only takes a few seconds for the solver to calculate the optimum proportions of TALs for each $\beta$ value. Next, the standard TA configurations are designed by minimizing the maximum $z$, the same objective in Model (8.8), and all the $\beta$ values. Note that while the $\beta$ value is 0 , all the five TAs are separate in the standard TA configuration, and the equivalent solution in the overlapping TAL scheme adapts all the UEs in each TA to contain only that TA in their lists. On the other extreme, when the $\beta$ value is maximum, all the five TAs are merged into one in the standard TA configuration. The overlapping TAL, in this case, gives a list containing all the five TAs to every UE. Hence, for the two extremes of the $\beta$ the optimal standard TA scheme and the overlapping TAL coincide.

The comparison of the standard TA scheme and the overlapping TAL for the baseline is illustrated in Figure 8.3. As it is explained above, the graphs coincide at the two ends. The figure shows that the standard TA scheme is changed in four discrete steps. By increasing the limit on the paging overhead, the TAs tend to merge. The behavior of the overlapping TAL scheme is smooth as the proportions of each TAL can be continuously changed. By allowing $10 \%$ addition to the paging overhead, the maximum TAU overhead 


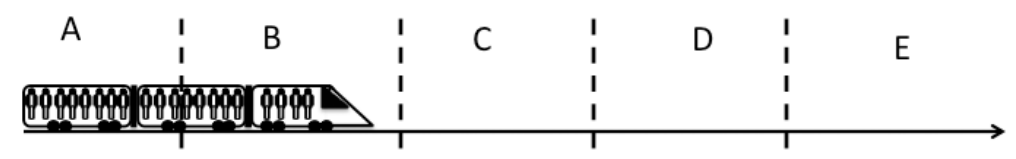

Figure 8.4: Congestion Scenario 1.

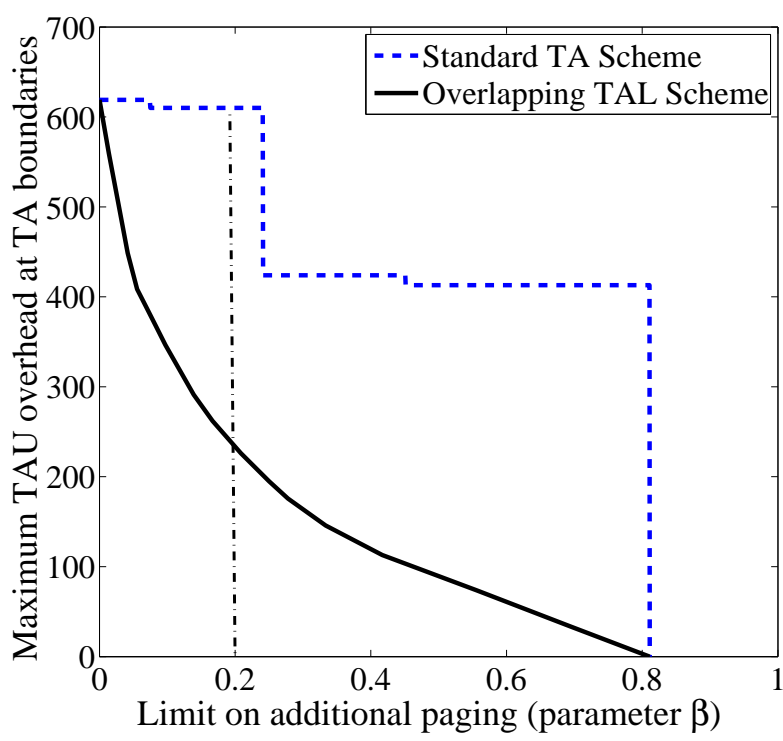

Figure 8.5: Evaluating the two schemes for Scenario 1.

at the TA boundaries is reduced by more than $50 \%$ with the overlapping TAL scheme, while the standard TA scheme can only reduce it by $20 \%$. Note that even in this uncongested scenario where mass mobility is not considered, the overlapping TAL scheme can significantly perform better than the standard TA scheme by overcoming the transitive restriction explained in Section 8.1. 


\subsubsection{Congestion Scenario 1}

In the first congestion scenario shown in Figure 8.4, a train moves from $A$ to $E$ having 600 passengers on-board. ${ }^{1}$ After a main station in $C$, the number of on-board passengers is reduced to 400 . It takes around half an hour for the train to pass the whole path (ignoring the time spent at the main station).

The load and handover data from the train scenario are aggregated and added it to the baseline scenario. All the UEs in the train passing each TA boundary will be added to the corresponding handover. The load is increased proportionally to the amount of time the train is spent in each TA. For example, the train spends about $6 \mathrm{~min}(0.1$ hour $)$ in $A$, therefore the load of $A$ is increased by $600 * 0.1=60$, due to the fact that the handover and load data are given for one hour period of time.

For various $\beta$ values, Figure 8.5 illustrates the behavior of the two schemes in minimizing the maximum TAU. Here the $\beta$ refers to the additional paging overhead due to the standard TA configurations and the proportions of the lists in the overlapping TALs compared to the original ones. By comparing the values on the y-axis in Figures 8.3 and 8.5, it can be concluded that Scenario 1 is highly congested. When the $\beta$ is equal to $20 \%$ of the total paging overhead, equivalent to 143.94 in Scenario 1 (shown by the vertical line in Figure 8.5), the standard TA scheme can reduce the maximum TAU signaling at the TAU boundaries by $0.01 \%$. In the same situation, the overlapping TAL scheme reduces the maximum TAU by $60.9 \%$. This case study demonstrates the benefit of the overlapping TAL scheme in mitigating the congestion problem of the train scenario.

\subsubsection{Congestion Scenario 2}

In Scenario 2, another train is added in the opposite direction to Scenario 1 (Figure 8.6). This train, which can be a regional train with speed reaching $160 \mathrm{~km} / \mathrm{h}$, has 450 passengers in the beginning and it stops in every TA on its way. It takes one hour for the train to pass the specified railway path. The same data aggregation is applied for this scenario.

Figure 8.7 illustrates the performance of the two schemes for Scenario 2. The results of Scenario 1 in Figure 8.5 are replicated to Figure 8.7 to ease comparison. Couple of observations follow from Figure 8.7. First by adding the second train to the scenario, the gap between the standard TA schemes

\footnotetext{
${ }^{1}$ One example can be TGV Eurostar train which can move at a speed up to $300 \mathrm{~km} / \mathrm{h}$ and may carry up to 784 passengers [78].
} 


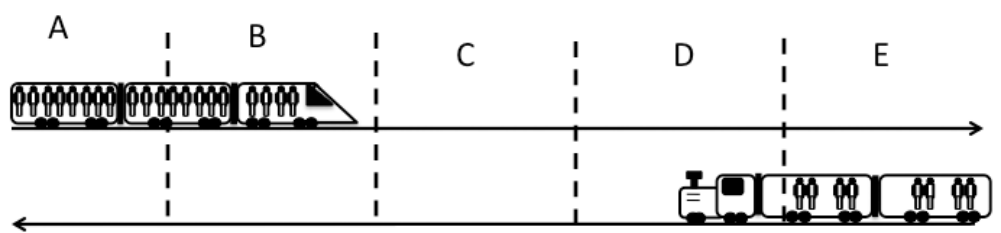

Figure 8.6: Congestion Scenario 2.

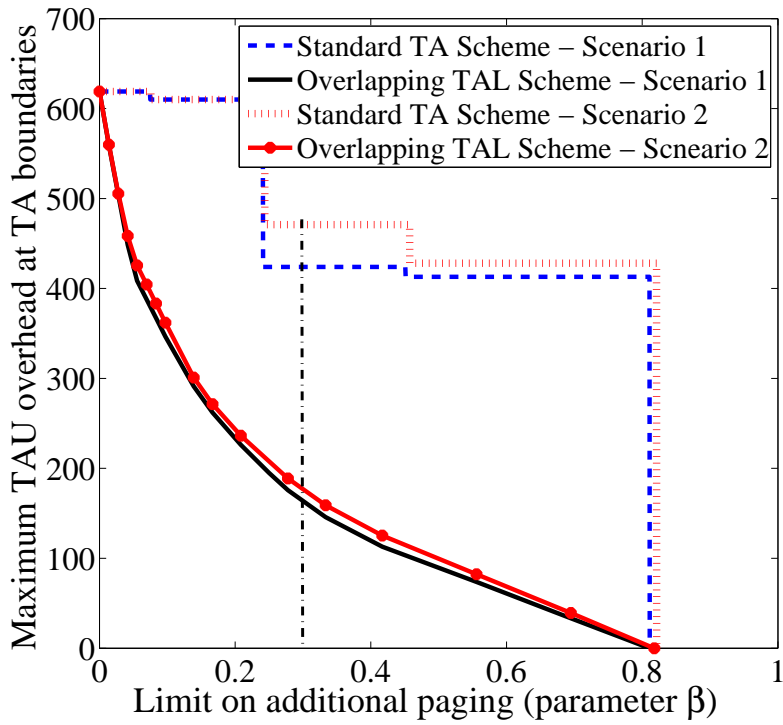

Figure 8.7: Evaluating the two schemes for Scenarios 1 and 2.

of Scenario 1 and 2 in some parts is more than ten times bigger than the corresponding gap for the overlapping TAL scheme. The proportions of TAL in reducing the maximum TAU overhead are mostly based on the largest amount of simultaneous movements of the UEs. Second, the overlapping TAL scheme performs much better than the standard TA scheme while mass mobility in both directions is present.

Let us consider $\beta=0.3$, equivalent to 215.91 (shown by the vertical line in Figure 8.7). The optimal standard TA configuration based on Model (8.8) is $\{(A, B, C),(D),(E)\}$, which can be represented by $x_{A C}=1, x_{D D}=1$ and $x_{E E}=1$ for both scenarios. However, the optimal TAL proportions in the overlapping TAL scheme for the two scenarios are given in Table 8.4. The 
variables' representations are based on the definition illustrated in Figure 8.2. Variables not included in the table are zero.

Table 8.4: Optimal solutions for $\beta=0.3$.

\begin{tabular}{ccccc}
\hline & \multicolumn{2}{c}{ Scenario 1 } & \multicolumn{2}{c}{ Scenario 2 } \\
& STA & OTAL & STA & OTAL \\
$\mathrm{x}_{A A}$ & 0 & 0.27 & 0 & 0.29 \\
$\mathrm{x}_{D D}$ & 1 & 0 & 1 & 0 \\
$\mathrm{x}_{E E}$ & 1 & 0.18 & 1 & 0.41 \\
$\mathrm{x}_{A B}$ & 0 & 0.26 & 0 & 0.24 \\
$\mathrm{x}_{A C}$ & 1 & 0.39 & 1 & 0.42 \\
$\mathrm{x}_{A D}$ & 0 & 0.08 & 0 & 0.05 \\
$\mathrm{x}_{B D}$ & 0 & 0.05 & 0 & 0.25 \\
$\mathrm{x}_{C D}$ & 0 & 0.26 & 0 & 0.11 \\
$\mathrm{x}_{C E}$ & 0 & 0 & 0 & 0.17 \\
$\mathrm{x}_{D E}$ & 0 & 0.60 & 0 & 0.37 \\
\hline
\end{tabular}

The overhead results of these configurations are given in Tables 8.5 for the two scenarios. As the TAL variables can take fractional values, the overlapping TAL scheme is able to utilize all the possible total additional paging (TPAG) to the favor of reducing the maximum TAU (MTAU), i.e. TPAG= $\beta C^{P}$ for the overlapping TAL scheme in the table. The numerical results show that the overlapping TAL scheme is able to mitigate the first congestion scenario by $61.49 \%$ and the second congestion scenario by $62.51 \%$ more than the standard TA scheme for the limit under consideration, which is $\beta=0.3$. The fundamental flexibility of the overlapping TAL scheme in assigning different proportions of each TAL significantly improves the performance compared to the standard TA scheme.

Table 8.5: Signaling overhead results for $\beta=0.3$.

\begin{tabular}{ccccc}
\hline & \multicolumn{2}{c}{ Scenario 1 } & \multicolumn{2}{c}{ Scenario 2 } \\
& STA & OTAL & STA & OTAL \\
$\beta C^{P}$ & 215.91 & 215.91 & 215.91 & 215.91 \\
MTAU & 424.00 & 163.28 & 471.00 & 176.59 \\
TPAG & 173.55 & 215.91 & 175.49 & 215.91 \\
\hline
\end{tabular}




\subsection{Conclusions}

In this chapter, a low-complexity solution approach was presented based on linear programming, to find the optimal proportional use of the lists of TAs for the proposed overlapping TAL scheme. The congestion mitigation effect was achieved by minimizing the maximum TAU signaling overhead in the network. The study showed that by assigning different TALs to the UEs involved in massive mobility, one can avoid the excessive TAU signaling messages at the TA boundaries. The transitive property of the standard TA configuration is relaxed in the overlapping TAL scheme, which considerably improves the load balancing capability.

There are several topics deserving further research. One is to apply the overlapping TAL scheme in a large-scale network to see its potentials compared to the standard TA scheme. This is studied in the following chapter. Another interesting subject worth studying is to extend the scheme by considering more data in hand, for example assuming that the destination of each UE in a train is known. 



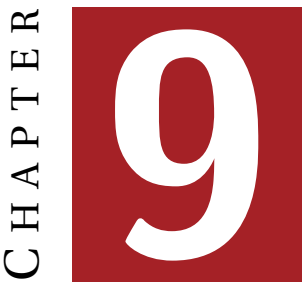

\section{Signaling Congestion Mitigation}

The rationale of avoiding signaling congestion is to ensure no significant degradation in the quality of service, which may occur due to resource exhaustion for tracking UEs. In addition to the train scenario explained in the previous chapter, there are several types of mobility patterns that may cause TAU and paging congestion. As explained in Chapter 3 , reconfiguring TAs in order to adapt to the UEs movement behavior during peak hours typically requires temporally turning down the cells, which is a costly process from the service perspective. This chapter aims to mitigate the signaling congestion resulted from mass mobility of idle UEs without performing a TA revision.

In previous studies of applying TALs, e.g., [35, 81, 118], the performance comparison relies heavily on the assumptions made on UE mobility and other network parameters. Because UEs that are initially registered to the same TAL by a cell will spread out within the area defined by the TAL, after some time duration, as a result of each UE's individual movement, the proportion of UEs assigned a certain TAL will become heterogeneous over the area. Note that the amount of TAU and paging generated by a UE are both dependent on the content of its TAL. Therefore, unless a very accurate mobility model is available (which is hardly the case in many scenarios), the practical performance of TAL may deviate significantly from the estimated one.

In the overlapping TAL scheme explained in the previous chapter, the construction of the LPs ensures the same proportional use of each TAL by all cells within the TAL. Therefore, statistically the proportion of UEs of each TAL remains homogeneous with the TAL's area, meaning that the performance is insensitive to individual UE movement. By assuming that the loca- 
tion distribution and mobility pattern of idle UEs are similar to those of active UEs, which is a well-accepted assumption, the performance of the overlapping TAL scheme is conveniently assessed. This makes the performance comparison both simple and unbiased, and its validity is not impacted by mobility models.

In this chapter, the LP formulation of the overlapping TAL scheme for the train scenario is generalized to find the optimal proportional use of the overlapping TALs for mitigating both TAU and paging congestions in largescale networks. The study of this chapter has previously appeared in [86].

\subsection{An Instructive Example}

In this section, the overlapping TAL scheme for TAU congestion mitigation is explained using a small example of a network with four TAs in Figure 9.1. Each of these TAs contain multiple cells and the assumption is that the TA layout in Figure 9.1 is optimum in terms of the total signaling overhead of the standard TA scheme. Suppose TALs which may partially overlap in their composition are created and assigned to UEs. Overall for the example in Figure 9.1, there are a total of fifteen TALs, each being a non-empty subset of $\{1,2,3,4\}$.

Any UE in this network will be registered to one of these fifteen TALs and each TA belongs to its respective collections of TALs. For example, the cells in TA 1 potentially belong to eight TALs, which can be assigned to the UEs registered or updated in $1:\{1\},\{1,2\},\{1,3\},\{1,4\},\{1,2,3\},\{1,2,4\},\{1,3,4\}$, $\{1,2,3,4\}$. Each of these eight TALs has a proportional value of usage, and the sum of all these values is one. For example TAL $\{1,3\}$ can be assigned to a UE from cells of both TAs 1 and 3. If TAs 1 and 3 assign different proportional values of this TAL to their UEs, then the total number of UEs being registered to the TAL in the area of these two TAs will depend on the UEs mobility behaviors. It is assumed that, the two TAs apply the same proportional values for the TALs containing both TAs. This assumption is generalized to any TAL, meaning that all TAs within the TAL are fully consistent in their proportional use of the TAL.

To formalize the optimization framework, the proportional usage of TAL $\ell$ is denoted by a non-negative optimization variable $x_{\ell}$. All cells in one TA will apply the same collection of TALs, and they update their UEs with the same proportional use of each TAL. For the network in Figure 9.1, the four TAs apply their respective collections of TALs by Equations (9.1)-(9.4). Because the usage of any TAL $\ell$ is unified within the entire service area of $\ell$, 


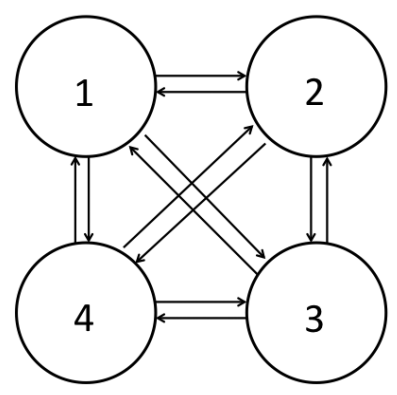

Figure 9.1: A small network with four TAs.

no matter if the UEs are currently stationary or making handover between cells in $\ell, x_{\ell}$ accurately provides the proportion of users being registered to $\ell$ among all UEs in the area. Note that these UEs could have received $\ell$ from any of the TAs in the list.

$$
\begin{aligned}
& x_{1}+x_{12}+x_{13}+x_{14}+x_{123}+x_{124}+x_{134}+x_{1234}=1 \\
& x_{2}+x_{12}+x_{23}+x_{24}+x_{123}+x_{124}+x_{234}+x_{1234}=1 \\
& x_{3}+x_{13}+x_{23}+x_{34}+x_{123}+x_{134}+x_{234}+x_{1234}=1 \\
& x_{4}+x_{14}+x_{24}+x_{34}+x_{124}+x_{134}+x_{234}+x_{1234}=1
\end{aligned}
$$

To calculate the number of TAUs from 1 to 3 and the opposite direction, the aggregated handover statistics of cells in 1 and 3, denoting them by $h_{13}$ and $h_{31}$ are required. Among the UEs making handover in the direction $1 \rightarrow 3$, only the UEs which do not have 3 in their TAL make a TAU. Therefore, the TAU overhead is $c^{u} h_{13}\left(x_{1}+x_{12}+x_{14}+x_{124}\right)$, and with the same reasoning the TAU overhead is $c^{u} h_{31}\left(x_{3}+x_{23}+x_{34}+x_{234}\right)$ for the opposite direction. The total TAU between the two TAs with the overlapping TAL scheme is $c^{u} h_{13}\left(x_{1}+x_{12}+x_{14}+x_{124}\right)+c^{u} h_{31}\left(x_{3}+x_{23}+x_{34}+x_{234}\right)$.

As all $x$-variables are between zero and one, the TAU may attain values in the range $\left[0, c^{u}\left(h_{13}+h_{31}\right)\right]$. Recall that, the total TAU between the two TAs is either zero or $c^{u}\left(h_{13}+h_{31}\right)$ in the standard TA scheme. As the overlapping TAL scheme is not constrained to satisfy the transitivity property, it has the potential to mitigate the congestion due to TAU signaling overhead. 


\subsection{Congestion Mitigation of TAU}

In this section, the optimization approach which was previously used for TAU congestion mitigation of the train scenario is generalized by optimizing the TALs for a network region where massive TAU is of concern.

All cells in one TA will use the same collection of TALs, and they update their UEs with the same proportional use of each TAL. The standard TA design is considered as the underlying structure. For the network region in question, denote the current TAs forming the region by set $\mathscr{T}=\{1, \ldots, T\}$. Each TA $v \in \mathscr{T}$ is a set of cells. The TAs can be, for example, optimized for the total TAU and paging overhead.

A min-max approach for TAU congestion mitigation is applied, that is, TALs are used to minimize the maximum TAU between all pairs of TAs. To this end, an upper bound on the increase of the total paging overhead is necessary, as otherwise the optimum would be to completely eliminate TAU by exclusively using the TAL containing all TAs. The bound is expressed as $\beta C^{P}$, where $C^{P}$ is the total paging overhead of TA design $\mathscr{T}$, and $\beta$ is a nonnegative parameter denoting the allowed percentage of increase.

The set of all possible TALs of $\mathscr{T}$ is denoted by $\mathscr{L}$. Thus $\mathscr{L}$ is the set of all non-empty subsets of $\mathscr{T}$. The total load parameter of all cells inside TA $v \in \mathscr{T}$ is given by $u_{v}$, and $h_{v w}$ denotes the total handover between TAs $v$ and $w$. These values are easily derived from the load and handover values between cells. Parameter $a_{v \ell}$ is a binary indicator, denoting whether or not TA $v$ is in TAL $\ell$. Moreover, $n_{v}$ denotes the number of cells in TA $v$. The aforementioned continuous variable $x_{\ell}$ represents the proportional use of TAL $\ell$ by all cells of the TAs in $\ell$. An auxiliary variable $z$ is used to denote the maximum TAU between TAs. The min-max TAU optimization problem can then be modeled by the following LP.

$\min z$

s.t. $\sum_{\ell \in \mathscr{L}} a_{v \ell} x_{\ell}=1, v \in \mathscr{T}$

$$
\begin{aligned}
& c^{u}\left(h_{v w} \sum_{\ell \in \mathscr{L}} a_{v \ell}\left(1-a_{w \ell}\right) x_{\ell}+h_{w v} \sum_{\ell \in \mathscr{L}} a_{w \ell}\left(1-a_{v \ell}\right) x_{\ell}\right) \leq z, v, w \in \mathscr{T}, v \neq w \\
& \alpha c^{p} \sum_{\ell \in \mathscr{L}} \sum_{\nu \in \ell} \sum_{w \in \ell, v \neq w} u_{v} n_{w} x_{\ell} \leq \beta C^{P}, \\
& x_{\ell} \geq 0, \quad \ell \in \mathscr{L} .
\end{aligned}
$$

The first constraint set (9.5b) assures that, within any TA, the propor- 
tional usage values of TALs containing the TA sum up to one. This is the generalization of (9.1)-(9.4). The next set of constraints (9.5c) defines the maximum TAU between TA pairs. For each ordered pair $v$ and $w$, there is a contribution of $c^{u} h_{v w} x_{\ell}$, if TAL $\ell$ contains $v$ (i.e., $a_{v \ell}=1$ ) but not $w$ (i.e., $a_{w \ell}=0$ ). Constraints (9.5d) bound the total paging overhead. For any list $\ell$, the overhead imposed by paging UEs in TA $v \in \ell$ to TA $w \in \ell$ is $\alpha c^{p} u_{v}$, scaled by the number of cells in $w$ and variable $x_{\ell}$ that stands for the proportional use of $\ell$.

It is often the fact that at the optimum of (9.5) the $x$-variables are fractional. As a result, some but not all of the UEs moving from one TA to another generate TAUs, in contrast to the original TA layout. In other words, the sharp boundaries between the TAs are "blurred" by the fractional use of TALs.

The number of variables in (9.5) is exponential in the number of TAs $T$. However, in practice this is not problematic for two reasons. First, the region with high risk of excessive TAU is typically confined to a part of the network. Second, as (9.5) is an LP, it can be solved efficiently even for a large number of variables.

\subsection{Congestion Mitigation of Paging}

The min-max optimization approach is not limited to congestion mitigation of TAU. It is easily adapted to reducing the risk of signaling congestion in paging that may become of concern for scenarios where massive UEs are populated densely in some area with little mobility (e.g., a football stadium). The paging overhead in this area becomes considerably higher than that in the rest of the network.

To reduce the paging overhead, some type of area splitting needs to take place. However, if the area would be in one common TA, such a split would not be possible without reconfiguring the TAs into smaller ones. For clarity of the discussion, every single cell is assumed to be a TA in the current configuration. The assumption does not impose excessive TAU, because the effect of "TA" of any size equivalently can be achieved by using a TAL containing the corresponding single-cell TAs [35, 82]. Thus, in this section, it is assumed that TALs are already in place in the current TA design, but the function has been limited to forming TAs in a conventional sense. For mitigating paging congestion, new (and smaller) TALs are created within the original ones, and their proportional usage levels are determined by the min-max optimization. 
Let $\mathscr{T}=\{1, \ldots, T\}$ be the set of single-cell TAs in the TAL containing the area with risk of paging congestion. As in the previous section, $\mathscr{L}$ is used to denote the non-empty collections of elements of $\mathscr{T}$. The other variable and parameter definitions are reused as well. Note that within $\mathscr{T}$, TAU is zero for the mentioned configuration (single TAL containing all TAs of $\mathscr{T}$ ). Reducing paging would lead to a positive amount of TAU, and the limit is denoted by $C_{\lim }^{U}$. The min-max LP formulation of paging congestion mitigation is provided in (7.1).

$\min z$

s.t. $\sum_{\ell \in \mathscr{L}} a_{\nu \ell} x_{\ell}=1, v \in \mathscr{T}$

$$
\alpha c^{p} \sum_{\nu \in \mathscr{T}} u_{v} \sum_{\ell \in \mathscr{L}} a_{\nu \ell} a_{w \ell} x_{\ell} \leq z, w \in \mathscr{T}
$$

$\sum_{v \in \mathscr{T}} \sum_{w \in \mathscr{T}: v \neq w} c^{u}\left(h_{v w} \sum_{\ell \in \mathscr{L}} a_{\nu \ell}\left(1-a_{w \ell}\right) x_{\ell}+h_{w v} \sum_{\ell \in \mathscr{L}} a_{w \ell}\left(1-a_{v \ell}\right) x_{\ell}\right) \leq C_{\lim }^{U}$,

$$
x_{\ell} \geq 0, \ell \in \mathscr{L} .
$$

The first set of constraints are identical to that of (9.5). Constraints (9.6c) define, together with the objective, the maximum paging. For each singlecell TA $w$, the left-hand side of (9.6c) represents the total amount of overhead generated to $w$ because of paging UEs located in TAs other than $w$, due to the use of TALs with multiple cells. The next constraint set is the aggregated form of $(9.5 \mathrm{c})$ to represent the total TAU, bounded by the limit value in the right-hand side.

The fixed limit value in (9.6) and parameter $\beta$ in (9.5) are not necessarily considered constants prior to running TAL optimization. In fact, the LP can be solved repeatedly for a range of limit values. Doing so generates a set of candidate solutions representing various levels of the trade-off between congestion reduction of TAU and the price of more paging, and vice versa (cf. Pareto optimization).

\subsection{Performance Evaluation}

In the following performance evaluation, TA and TAL are defined on the site level and the overhead of a single TAU, $c^{u}$ is set ten times as much as $c^{p}$ [66]. Parameter $\alpha$ is set to 0.05 , i.e. $5 \%$ of the UEs are paged. Table 9.1 gives the numerical assumptions and values for this performance evaluation. 
For all the scenarios, there is an underlying TA layout (possibly implemented via TALs) that represents the most likely design in terms of planning. Namely, the TA layout is the global optimum in minimizing the total signaling overhead (i.e., $C^{P}+C^{U}$ ). This optimum TA layout is computed using the model in [109] and software CPLEX [60].

Table 9.1: Numerical assumptions and values for performance evaluation.

\begin{tabular}{lll}
\hline Parameter & Description & Values \\
$\alpha$ & Call intensity factor & 0.05 \\
$\beta$ & Allowed percentage increase on the total paging & {$[0,0.56]$} \\
$\sigma$ & Allowed increase ratio on the total TAU & {$[1,6.3]$} \\
$c^{p}$ & Cost of a single paging & 0.1 \\
$c^{u}$ & Cost of a single TAU & 1 \\
$C^{P}$ & Total paging overhead & 18603 \\
$C_{\max }^{P}$ & Maximum congestion in paging & 1058 \\
$C^{U}$ & Total TAU overhead & 23849 \\
$C_{\max }^{U}$ & Maximum congestion in TAU & 5996 \\
$C_{\lim }^{U}$ & Limit on additional increase in the total TAU & {$[23849,150249]$} \\
$N$ & Number of sites & 60 \\
$T$ & Number of TAs & 7 \\
\hline
\end{tabular}

Whereas comparing the performance of the proposed overlapping TAL scheme with the underlying TA design gives insights, it is somewhat biased to the favor of the former, because the latter is not optimized for signaling congestion but for the total signaling overhead. For this reason, the performance comparison is conduced between overlapping TALs and a reoptimization of TA layout for the objective metrics of this chapter, that is, the TAs can be merged for the objective function (9.5a) or split for the objective function (9.6a). This gives an unbiased assessment of optimized overlapping TALs.

The trade-off between the signaling overhead of TAU and paging has to be dealt with in both the standard TA scheme and the overlapping TAL scheme. For the latter scheme, reducing TAU congestion by using overlapping TALs results in overall higher paging in comparison to the baseline TA design, and vice versa. This aspect is reflected by the limit parameters in (9.5) and (9.6). For the limit parameter $\beta$ in (9.5), the minimum possible value is zero. In this case, no additional paging is permitted, and consequently the only feasible solution is to reuse the baseline TA design. That is, the standard TA scheme and overlapping TAL scheme coincide. When $\beta$ increases, the concern of paging overhead diminishes, and both schemes 
eventually coincide again, giving the same solution with one single TA (or TAL) containing all sites with zero TAU overhead. At this point, constraint (9.5d) is void, and increasing $\beta$ further clearly has no effect. A similar observation applies to the TAU limit in (9.6). Namely, the two schemes coincide when the limit is zero for which the baseline TA design is kept, or when the limit is very large such that the optimum for both is to split TAs maximally with zero paging (but completely sacrifices TAU). Thus, for each of the limit parameters, there is a maximum value of significance.

In the performance evaluation, for each limit parameter, its maximum value of significance is found by using bi-section search for the given planning scenario. Then, within the range of significance, computations for 500 equally-spaced values of the limit parameter are conducted, in order to give a comprehensive performance picture. The results represent, in fact, a large number of possible options for the trade-off between TAU and paging, both for the standard TA scheme and for the overlapping TAL scheme, allowing the network operator to select the solution to implement, based on the operator's preference.

The data from the Lisbon network is again used for performance evaluation on a general and large-scale topology. Figure 9.2 illustrates the underlying TA layout (i.e., the optimum with respect to the total signaling overhead). There are seven TAs in the design; these are used for creating overlapping TALs. Note that, in mitigating signaling congestion of paging, the functionality of the standard TA scheme is implemented with non-overlapping TALs of single-sited TAs (see Section 9.3). In this case, the term TA group is used for the sake of clarity.

Key statistics of the TA design are as follows. The total TAU overhead is $C^{U}=23849$, and the total paging overhead is $C^{P}=18603$. The highest TAU between TAs, denoted by $C_{\max }^{U}=5996$, and the most congested site in paging has a paging overhead of $C_{\max }^{P}=1058$.

For parameter $\beta$, the range of significance in this case is $[0,0.56]$. Formulation (9.5) in Section 9.2 is solved with $\beta$ varying within the range. The same range is applied in re-optimizing TAs with the standard TA scheme for the same objective function (9.5a). The results are displayed in Figure 9.3 and Table 9.2. In addition to the signaling overhead, the table contains information of the computing time.

As was previously explained in Chapter 8 for the case of train scenario, the performance curves coincide at the two ends. One immediate observation is that the curve of the overlapping TAL scheme is smooth, whereas that of the standard TA scheme has a stepwise shape. For the latter, the TAs 


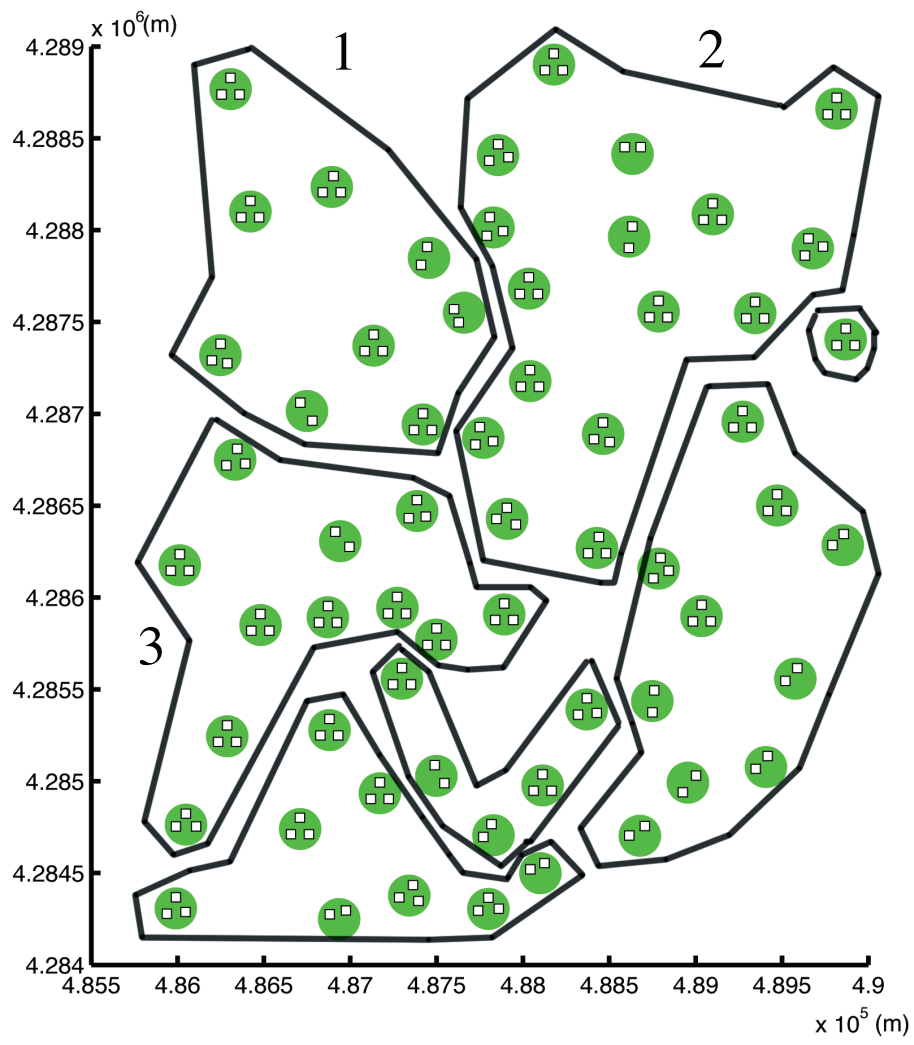

Figure 9.2: The underlying TA design.

Table 9.2: Performance comparison for TAU congestion mitigation.

\begin{tabular}{ccccc}
\hline$\beta$ & \multicolumn{2}{c}{ Standard TA scheme } & \multicolumn{2}{c}{ Overlapping TAL scheme } \\
& Maximum TAU & Time $(\mathrm{ms})$ & Maximum TAU & Time $(\mathrm{ms})$ \\
0.0 & 5963 & - & 5953 & 8 \\
0.1 & 4109 & 52 & 537 & 12 \\
0.2 & 2953 & 52 & 131 & 12 \\
0.3 & 2423 & 76 & 62 & 12 \\
0.4 & 1910 & 60 & 37 & 12 \\
0.5 & 373 & 230 & 14 & 12 \\
\hline
\end{tabular}




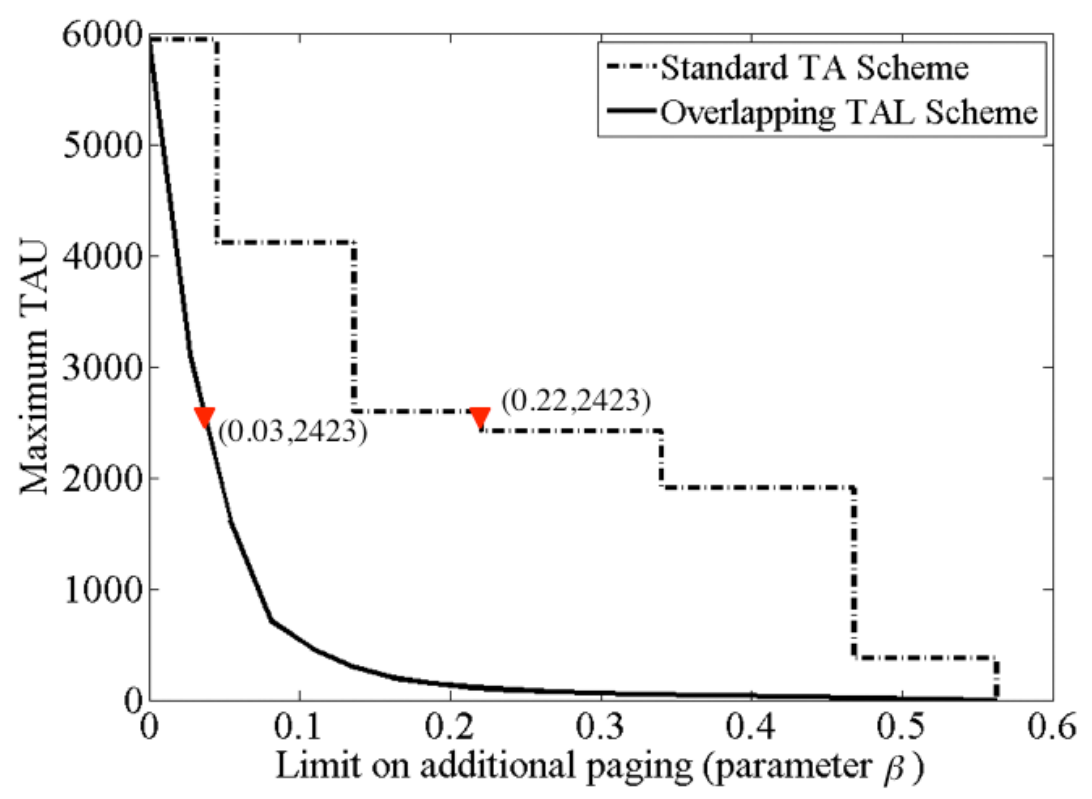

Figure 9.3: Evaluating the TAU congestion mitigation.

may not overlap due to the binary and transitive properties of the $\mathbf{S}(\mathbf{t})$ matrix (Section 5.1). As a result, the only possibility of reducing the maximum TAU is by merging TAs. This inherent limitation of the standard TA scheme leads to the stepwise shape. Overlapping TALs, in contrast, can achieve "partial merge" of TAs, by solving the optimization formulation (9.5), yielding a smooth curve that significantly outperforms the standard TA scheme.

For example, if the total paging overhead is allowed to increase by $10 \%$, re-optimizing TAs by the standard TA scheme reduces the peak TAU between TAs from $C_{\max }^{U}=5996$ to slightly above 4109 . Using optimized overlapping TALs, the peak is pushed down to 537, see Table 9.2. This is only one seventh of the maximum TAU resulted from the standard TA scheme. Hence, TAU signaling congestion can be effectively handled by the proposed TAL scheme with little price paid for a higher amount of paging.

Examining the computing time in Table 9.2 shows that solving (9.5) for TAU mitigation runs very fast, and the computing time is not sensitive at all to the limit value. In fact, the overlapping TAL scheme runs several times faster than the standard TA scheme. In conclusion, the former has superior performance both in TAU mitigation and the computational burden.

Figures 9.4 and 9.5 provide illustrations of the designs corresponding to 


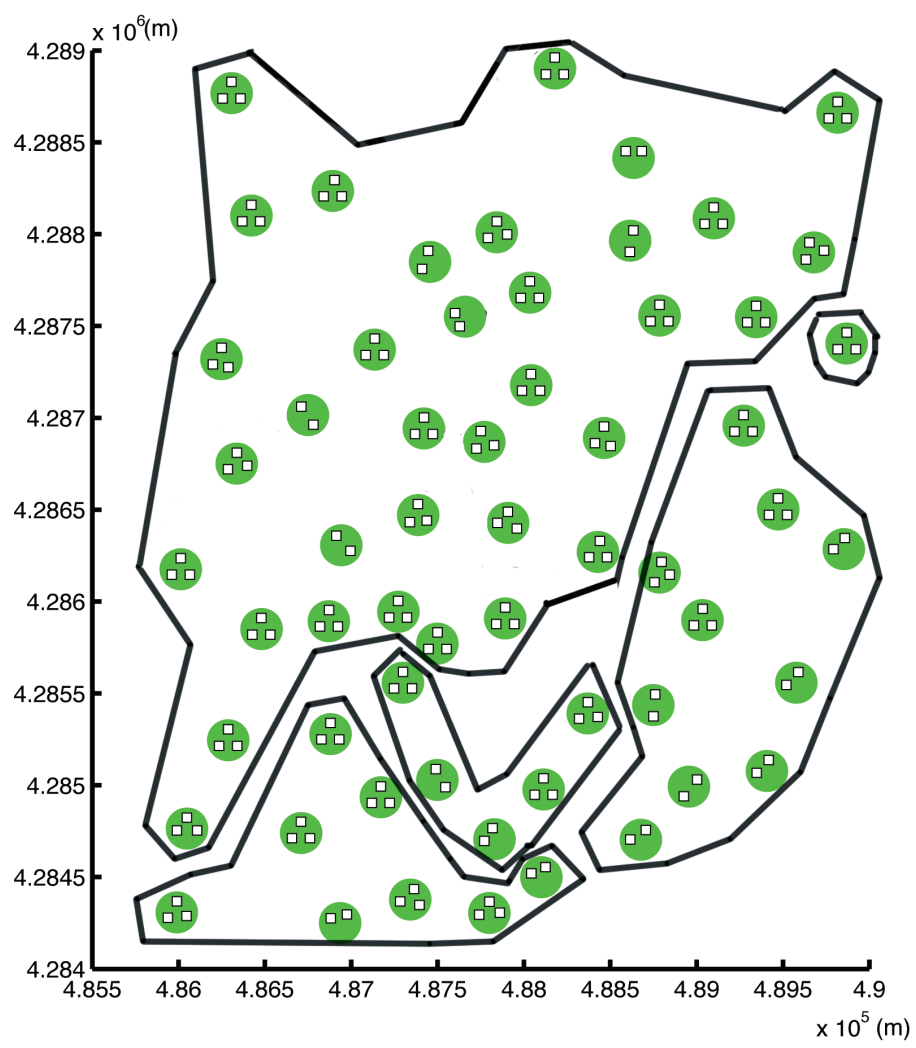

Figure 9.4: The standard TA design for a chosen maximum TAU.

the two markers in Figure 9.3. The standard TA scheme and overlapping TAL give the same level of peak TAU (approximately 2400) for the selected solutions, although the former has considerably higher paging. Figure 9.4 shows the TA layout given by the standard TA scheme; one can see this layout is a simple merge of TAs 1-3 in Figure 9.2. The composition and usage of TALs are shown in Figure 9.5. In this figure, the original three TAs are still used, but to lesser extent. In addition, TALs containing pairs of TAs are in place, with the effect of reducing TAU peak between them. The illustration highlights the effect of blurring TA boundaries by overlapping TALs.

As the last part of the performance evaluation, the overlapping TAL scheme is applied for mitigating paging congestion, using Figure 9.2 as the base lay- 


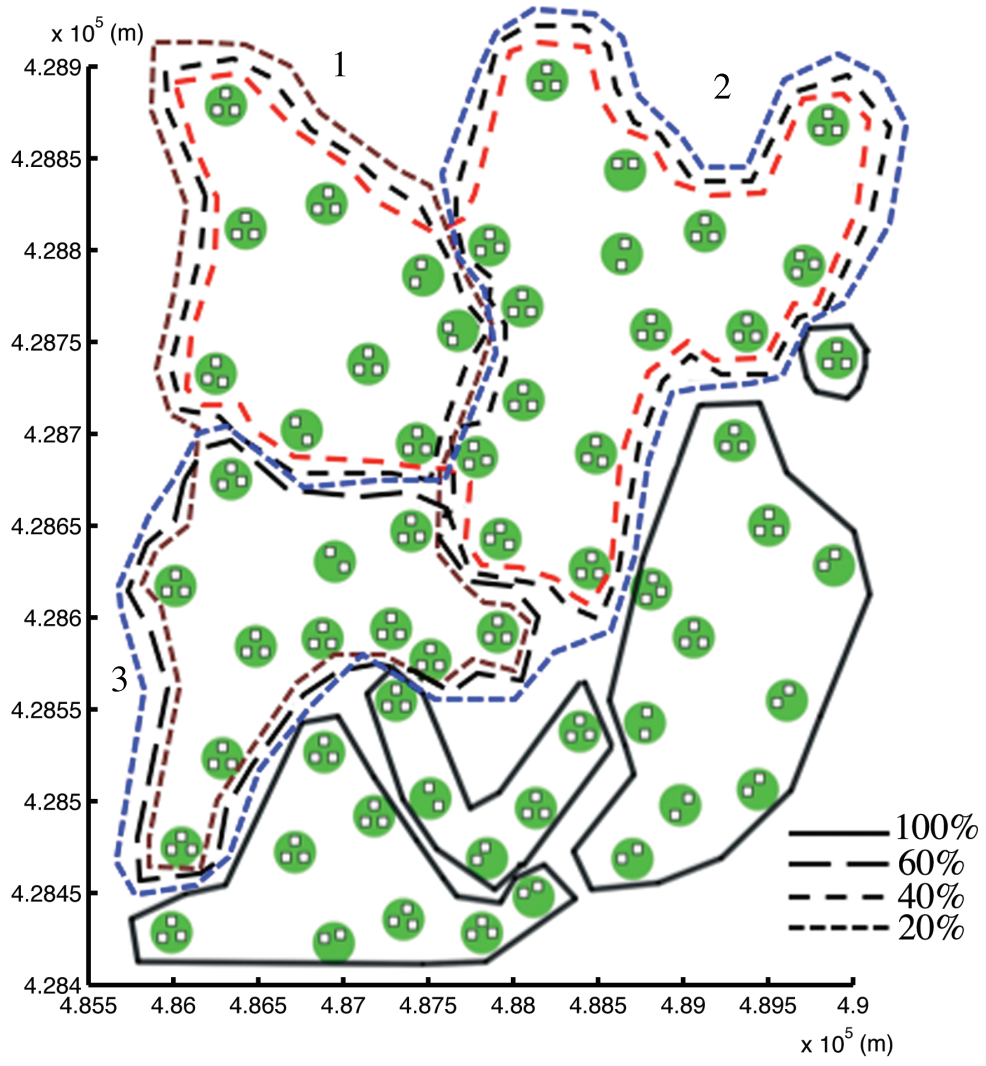

Figure 9.5: The overlapping TAL design for a chosen maximum TAU.

Table 9.3: Performance comparison for paging congestion mitigation.

\begin{tabular}{ccccc}
\hline$\sigma$ & \multicolumn{2}{c}{ Standard TA scheme } & \multicolumn{2}{c}{ Overlapping TAL scheme } \\
& Maximum paging & Time (s) & Maximum paging & Time (s) \\
1.0 & 1058 & - & 1058 & $<1$ \\
1.2 & 847 & 45 & 201 & 33 \\
1.4 & 501 & 555 & 72 & 94 \\
1.6 & 400 & 340 & 22 & 1881 \\
1.8 & 340 & 517 & 11 & 259 \\
2.0 & 324 & 378 & 6 & 384 \\
\hline
\end{tabular}




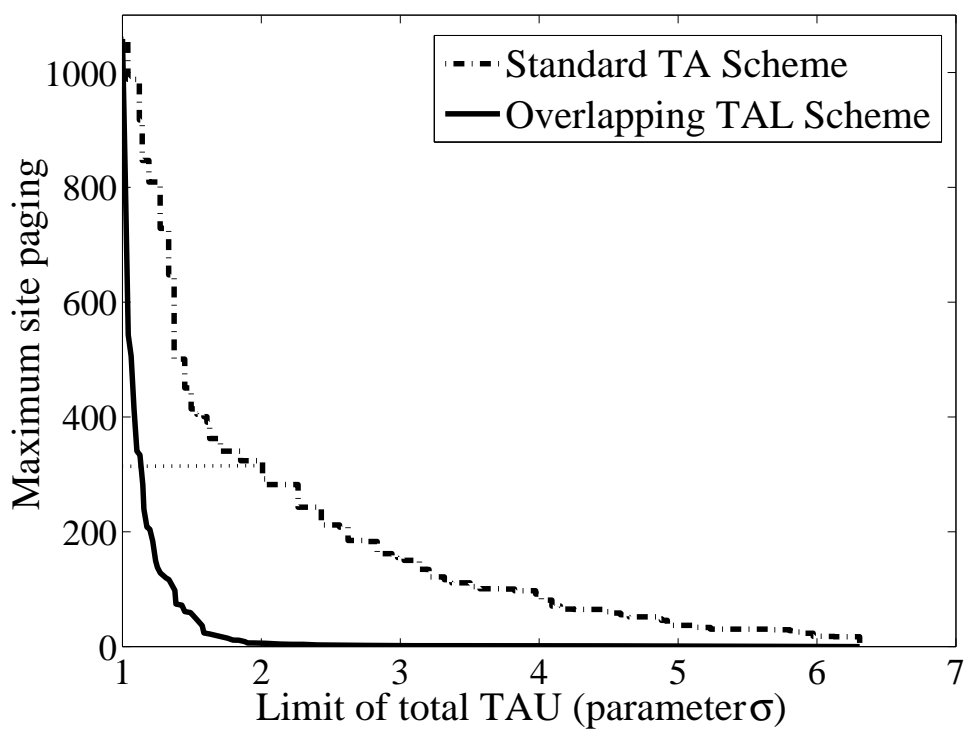

Figure 9.6: Evaluating the paging congestion mitigation.

out. In this case, the TAs are assumed to be single-sited, and the grouping in Figure 9.2 is implemented by means of non-overlapping TALs explained in Section 9.3. Overlapping TALs are then created and optimized by formulation (9.6) to achieve partial split within each TA group. The TA groups are also re-optimized following the standard principle of TA design; this corresponds to finding the optimal split (with sharp boundaries) for objective function (9.6a). The performance comparison is given in Figure 9.6 and Table 9.3. For the sake of compactness, the results represent the aggregated values over all TA groups. Thus $C_{\lim }^{U}$ can be set in relation to $C^{U}$ of the baseline design. The ratio between $C_{\lim }^{U}$ and $C^{U}$ is denoted by $\sigma$. The range of significance for the scenario is $\sigma \in[1.0,6.3]$.

In the figure, the horizontal axis is $\sigma$, For example, $\sigma=2.0$ on the axis means that $C_{\lim }^{U}=2 C^{U}$. The vertical axis represents the maximum paging overhead generated by the sites. For each site, this overhead is the amount of paging overhead of the site in order to locate UEs of other sites. When $\sigma=$ 1.0, the only feasible solution is to keep the baseline TA groups, for which the maximum site paging equals $C_{\max }^{P}$. The maximum decreases gradually in respect of $\sigma$, and reaches zero (i.e., full split) when $\sigma \geq 6$.3. Note that in Table 9.3, $\sigma$ is limited to 2.0 , because the maximum paging in the overlapping TAL 
scheme is virtually zero beyond this point.

In comparison to Figure 9.3, the performance curve of the standard TA scheme in Figure 9.6 is much smoother. This is because splitting a TA group can be done with a much better granularity (e.g., one site at a time) than merging TAs. Nevertheless, the performance is evidently inferior to that of adopting overlapping TALs, as can be seen from Figure 9.6 and Table 9.3. The overlapping TAL scheme is able to reduce the maximum paging much more steeply in comparison to conventional split of TA groups. For example, the former virtually eliminates paging, if the TAU is allowed to be doubled (i.e., $\sigma=2.0$ ), whereas in the latter case the maximum paging is still above one third of the baseline value, as shown in Table 9.3. Moreover, the magnitude of the performance difference grows in $\sigma$ for the range considered in the table. As another comparison, recall that the average site paging of the baseline design is approximately 310 (that is, $C^{P}$ divided by the number of sites), and suppose it is of interest to bring the maximum paging level down to this value, for which the horizontal dashed line in the figure show the performance of the two schemes. Using the standard TA scheme (or equivalently non-overlapping TALs), the TAU overhead has to be doubled. With optimized overlapping TALs, the effect is achieved with merely a $13 \%$ increase in TAU. Thus overlapping TALs provide an effective means of splitting hotspots for avoiding paging congestion.

In Table 9.3, the computing time used by the overlapping TAL scheme is up to 30 minutes. The major part of the time is attributed to the largest TA group with 16 sites, see Figure 9.2. For this TA group, the LP (9.6) contains $2^{16}-1$ variables. Although the run time may not admit on-line use, it remains reasonable in a planning stage, because typically a hotspot of massive and close-to-static UEs can be predicted in advance. It is also observable that time values for the overlapping TAL scheme and the standard TA scheme are comparable in magnitude, and the former scheme runs faster for all except for two cases in the table.

\subsection{Conclusions}

This chapter presented a scheme using a overlapping TALs and a min-max optimization approach for the purpose of mitigating signaling congestion for location management in LTE networks. The optimization task was formulated and computed by means of linear programming. As the key aspect of the optimization framework, consistency in the proportional use of TALs is maintained throughout the network, and consequently no assumption 
or model of UE mobility is required in order to assess the performance of overlapping TALs. Numerical evaluations of mitigating massive TAU as well as paging congestion demonstrated that the proposed optimization framework is very effective. Optimization of overlapping TALs significantly outperforms the standard TA scheme in signaling congestion. By providing high granularity in re-distributing the signaling overhead over the network area, TALs open up a new dimension of freedom in location management.

An interesting line of extension of the current work is dynamic configuration of overlapping TALs. By monitoring TAU and paging statistics and adapting the proportional use of TALs accordingly, the proposed TAL scheme can be extended to form an automated process in location management within the paradigm of self-optimization networks. This topic will be addressed in the next chapter. 



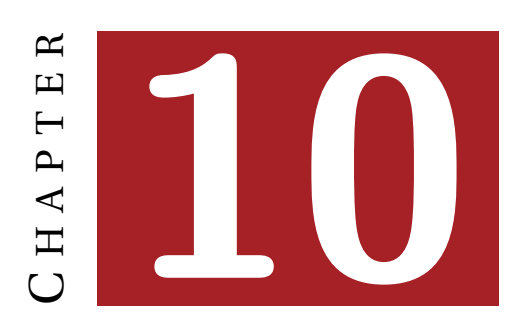

\section{STUDY OF STATIC AND DYNAMIC DESIGNS}

Conventionally, TAs are manually configured and the design is static. While network conditions change, this approach is not efficient for reconfiguring TAs. The static design often does not perform very well in terms of the signaling overhead, but it has the advantage of low computational complexity. In contrast, a dynamic approach that frequently updates the designs may achieve better results, while requiring a higher degree of computational effort $[39,47]$.

Self-Organizing Networks (SON) capability is one of the key components of the LTE network. Automatic dynamic configuration is a key aspect for SON. Although the concept of automated reconfiguration is not new in cellular networks and there are already extensive uses of automated processes in performance engineering, introducing a higher level of automation remains one of the key topics in cellular communications. In Releases 8 and 9, 3GPP is standardizing self-optimizing and self-organizing capabilities for LTE. This was a continuation of the natural evolution of automation in cellular networks by extending the scope deeper into the network for LTE [9]. SON techniques aim at doing both planning and reconfiguration in a semiautonomous fashion.

Due to the complexity of TA reconfiguration, until now the operators decide about the TA of each cell at the time of network deployment, and changes are made only in case of extreme performance degradations. The network continuously collects UE statistics and monitors performance indicators, and in SON there is a potential of improving the TA or TAL designs in short time intervals without any cost in terms of service interruption [97]. 
The aim of this chapter is to study the performance of the standard TA scheme under static and dynamic frameworks. The comparison of the two frameworks helps in understanding the potential of SON in reducing the OPEX of the network. Moreover, the problem of signaling congestion mitigation is studied in a dynamic framework. The dynamic framework is intended to correspond to SON mechanism. The work presented here has partially been published in [82].

\subsection{SON Dynamic Framework}

In a dynamic framework, the optimum configuration is always based on the data which has been collected until that specific point in time. However, the optimum design based on the gathered data up till that time, may not remain optimum for the next time interval. This is due to the dynamic change in UEs' behaviors. Hence, the challenge in the dynamic framework study is the signaling overhead estimation based on different sets of available data.

Previously in [82, 83], the challenge was tackled by defining different sets of signaling overhead values including ideal, potential and actual. The ideal/potential signaling overhead represented the signaling overhead of applying the STA/TAL design made for data of time interval $d$ to the same data. The actual signaling overhead, which was the most practically feasible estimation among all, represents the signaling overhead of applying the design made for data of time interval $d-1$ to the data of time interval $d$. However, in this case the design was optimized for one set of data and applied to the next set of data, therefore the approach was vulnerable to consecutive sets of data, and may not be practical for SON. In this section, a mechanism to use the data collected from SON is proposed for the design of standard TA and TAL layout in a dynamic framework. A realistic assumption considered in this study is that the similarity of data degrades by the increase of time interval.

The dynamic SON time framework is denoted by the set $\mathscr{D}=\{1, \ldots, D\}$, and $D$ is the total number of data sets. The data entering the optimization model $\bar{u}_{i}(d)$ and $\bar{h}_{i j}(d)$ are defined to be the weighted sum of the previous value and the new data gathered in the last time slot $u_{i}(d-1)$ and $h_{i j}(d-1)$. The idea is to smoothly reduce the impact of old data sets, and not making a sharp change in the design of two consecutive time slots. With each collected data from SON, $u_{i}(d-1)$ and $h_{i j}(d-1)$, the new aggregated $\bar{u}_{i}(d)$ and $\bar{h}_{i j}(d)$ are computed by Equations (10.1) and (10.2). These two equations are referred to as "exponential filters" in the glossary of statistical terms, 
where $\lambda$ is a constant between 0 and 1 that smooths the changes in $\bar{u}_{i}(d)$ and $\bar{h}_{i j}(d)$.

$$
\begin{gathered}
\bar{u}_{i}(d)=(1-\lambda) \bar{u}_{i}(d-1)+\lambda u_{i}(d-1) \\
\bar{h}_{i j}(d)=(1-\lambda) \bar{h}_{i j}(d-1)+\lambda h_{i j}(d-1)
\end{gathered}
$$

The total TAU and paging signaling overhead for the TA design of time slot $d, \mathbf{S}^{d}(\mathbf{t})$ are denoted by $C^{U}(d)$ and $C^{P}(d)$, respectively. Equations (10.3) and (10.4) compute the TAU and paging signaling overheads for each time slot $d$. If cells $i$ and $j$ are in different TAs, according to Equation (10.3) the movement between the two cells contributes to $C^{U}(d)$. Based on Equation (10.4), the total paging overhead generated by cell $i$ to other cells in the same TA is computed by the load of cell $i$ and the size of that TA. Note that a paging overhead is generated in each of the cells in the TA, except the cell where the UE is located. This is considered by the inner summation. By adding the paging overhead of all cells in the network (the outer summation), the total paging overhead is calculated for time slot $d$.

$$
\begin{gathered}
C^{U}(d)=\sum_{i \in \mathscr{N}} \sum_{j \in \mathscr{N}: j \neq i} c^{u} \bar{h}_{i j}(d)\left(1-s_{i j}^{d}(\mathbf{t})\right), d \in \mathscr{D} \\
C^{P}(d)=\sum_{i \in \mathcal{N}} \sum_{j \in \mathscr{N}: j \neq i} \alpha c^{p} \bar{u}_{i}(d) s_{i j}^{d}(\mathbf{t}), d \in \mathscr{D}
\end{gathered}
$$

\subsection{Congestion Mitigation in SON}

The SON mechanism can combine the static and dynamic framework based on the network behavior. The TAU and paging congestion problems do not exist all the time, but only during certain time intervals and locations. Hence, the standard TA scheme can be kept until the time when TAU congestion reaches a certain threshold. According to the study in Chapter 9, the objective of the overlapping TAL scheme is to minimize the maximum TAU overhead between the TA boundaries or paging overhead of a standard TA scheme. Thus based on the SON mechanism, whenever the maximum TAU signaling at the TA borders exceeds a pre-defined threshold value, say $\xi$, the overlapping TAL scheme is applied to reduce this value and to avoid the congestion in the network.

One major issue in applying the overlapping TAL scheme in a dynamic framework is making a reasonable assumption for the parameter $\beta$ in (9.5) 
and $\sigma$ in (9.6). A relatively high value for $\beta$ or $\sigma$ can completely eliminate the congestion problem, while it introduces a large amount of paging overhead or TAU overhead in each time interval, whereas $\beta$ or $\sigma$ of very small size might not solve the problem of congestion to the limit lower than $\xi$. This in fact opens a new problem which deserves further research.

An alternative in using the overlapping TAL scheme is the fact that the set $\mathscr{T}$ can denote the TAs inside the congested region and not the total TAs of the entire network. Therefore, one mechanism is that while MME monitors the TAU and paging overheads and determines the congestion area, it can allow only those TAs with high TAU or paging at their boundaries and their neighboring TAs to enter the optimization formulations (9.5) or (9.6), respectively. In this way the problem significantly reduces in size. However, this study explores the bigger picture and considers the entering of all the TAs of the network inside the optimization model and solves the problem of exponentially higher size. One main reason is that the congestion can happen in several parts of the network at the same time interval and as TAs are geographically connected to each other, solving the congestion problems individually for small subsets of TAs, may omit the optimum proportional use of TALs which could be found by solving the overall problem.

\subsection{Performance Evaluation}

The performance evaluation in this chapter consists of two parts. One is to compare static and SON dynamic framework for the standard TA scheme. The other one is to apply the overlapping TAL scheme in a dynamic framework, and evaluate its performance. The overlapping TAL scheme is studied on the problem of TAU signaling congestion, because based on the available data, the TAU overhead contributed more in the total signaling overhead. However, it is expected that the mitigation of paging signaling congestion results to a similar outcome.

In this study, the realistic data of the Lisbon network is again used, and the performance evaluation has been applied at site level. The generated traffic data is simulated based on the mobility patterns of the city streets and the traffic intensity of the region types (e.g. commercial, office, housing,...) differentiated at different times of the day and week. Due to a dynamic framework, for each time interval, there exists a set of site load and handover statistics, which are used as an approximation for idle UEs locations and mobility behavior. The time frame of one entire week is considered in the experiment and each time interval is one hour. Hence, the number of 


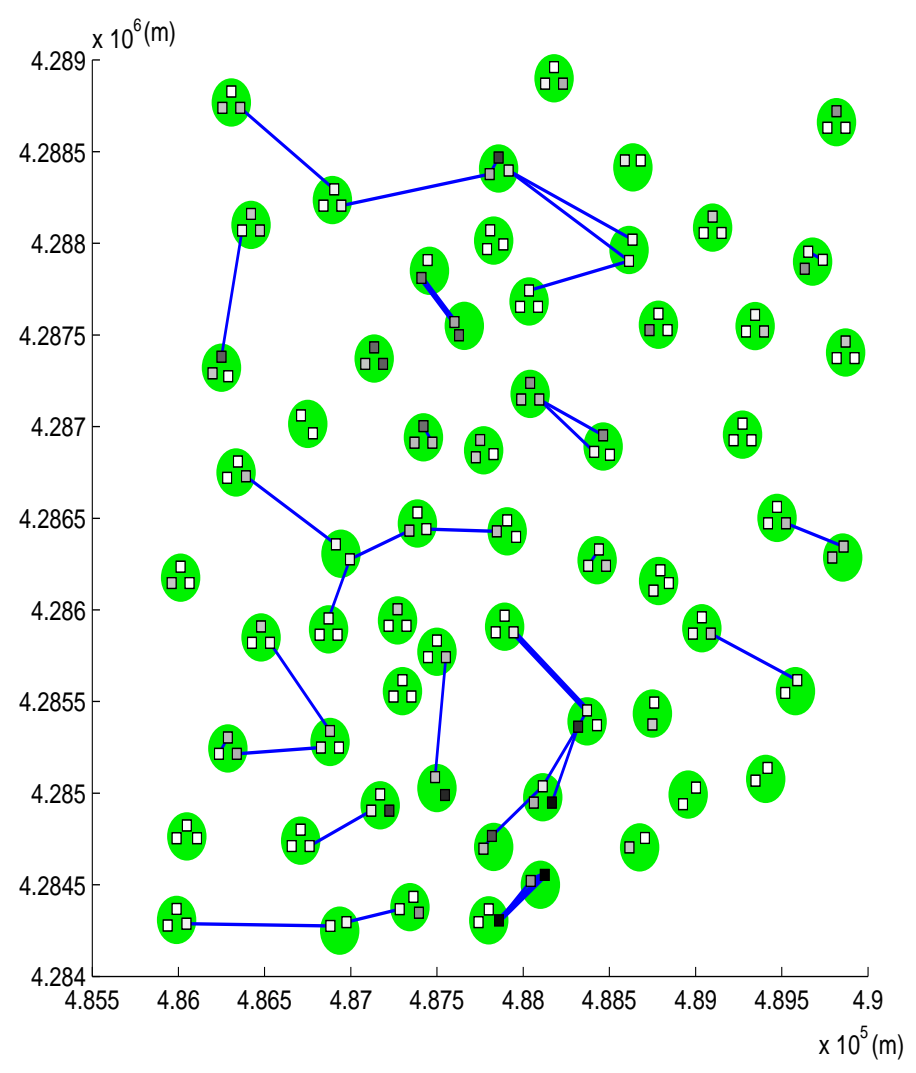

Figure 10.1: An illustration of the number of mobility in the Lisbon network on a Monday dawn (3:00-4:00).

data sets $D$ is 168 . The traffic is simulated to vary over different times of each day and different days of the week. This is especially observable when comparing the weekdays to weekends. The UEs' activities are very low during nights and higher during days with two peaks at the beginning and the end of the office hours. For example, Figure 10.1 illustrates a low number of mobility between (3:00-4:00) of a Monday morning, and Figure 10.2 shows a high number of mobility at (17:00-18:00) of a Friday afternoon in the Lisbon network. 


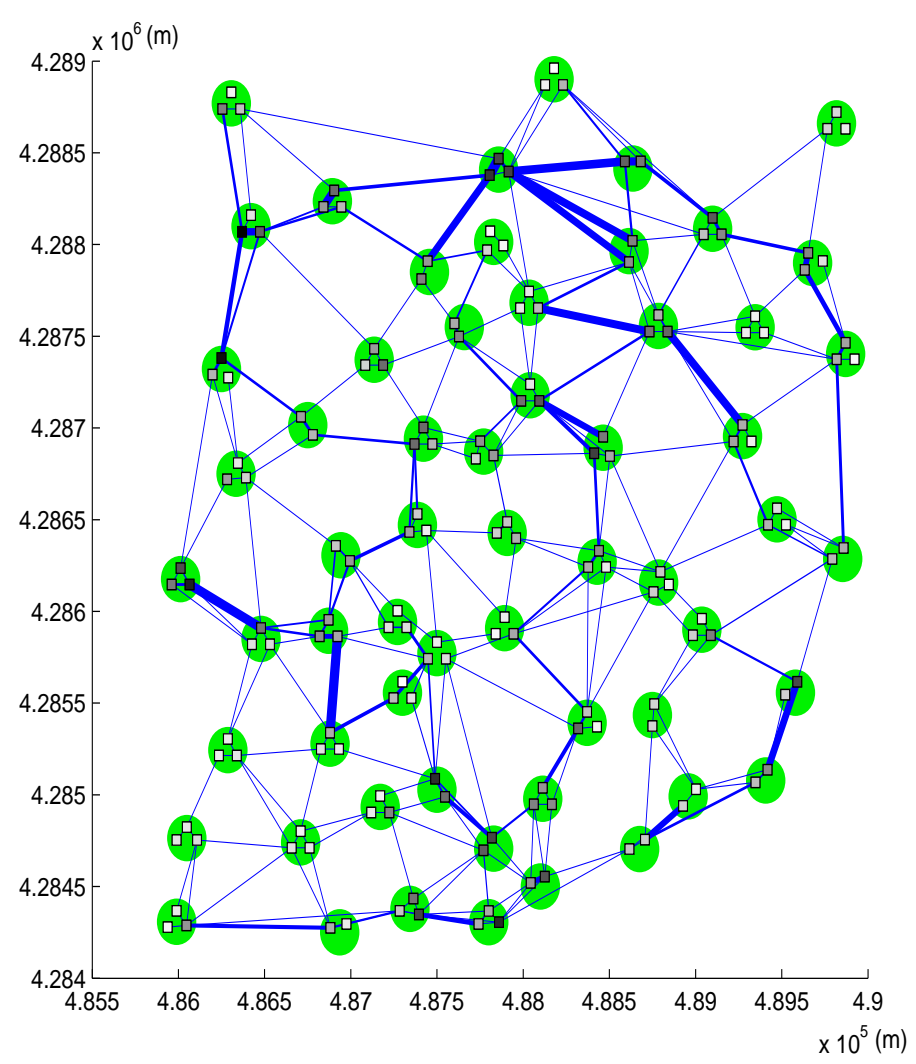

Figure 10.2: An illustration of the number of mobility in the Lisbon network on a Friday evening (17:00-18:00).

\subsubsection{A Comparison of the Static and SON Dynamic STA}

In the static TA, which is still the location management deployment in most cellular networks, a standard TA design based on the average data of site load and handover of the entire time period (in this case study: one week) is applied to all the data sets. The optimum standard TA design is computed by CPLEX [60] using the model in [109].

The dashed lines in Figures 10.3 and 10.4 illustrate the total and the TAU signaling overhead of the static standard TA scheme during the examined week. The values presented on the y-axis of all the forthcoming diagrams are all based on the same cost unit, and due to having the same unit, they 


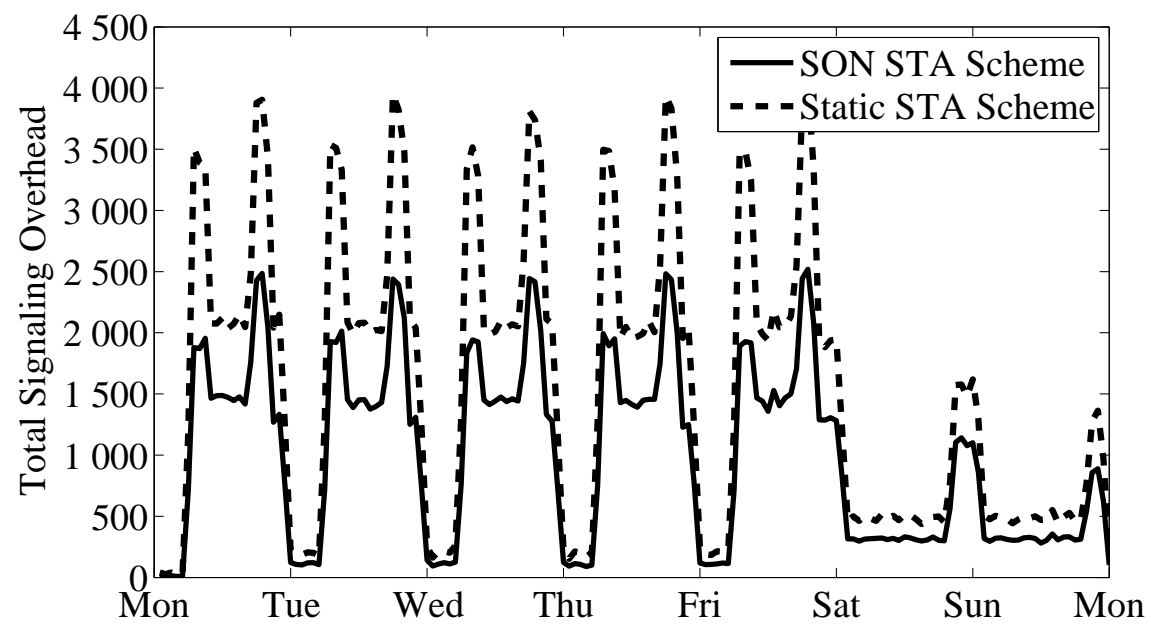

Figure 10.3: The total signaling overhead of the static and SON standard TA schemes.

are comparable. In the entire experiment, the call intensity factor $\alpha$ is set to 0.05 , and the overhead of a single TAU $c^{u}$ is set ten times as much as $c^{p}$ [66]. Table 10.1 summarizes all the numerical assumptions and values considered in the following performance evaluation study.

Table 10.1: Numerical assumptions and values for performance evaluation.

\begin{tabular}{lll}
\hline Parameter & Description & Values \\
$\alpha$ & Call intensity factor & 0.05 \\
$\beta$ & Allowed percentage increase on the total paging & {$[0.05,0.1]$} \\
$\lambda$ & Smoothing factor & 0.75 \\
$\xi$ & Paging threshold value & 100 \\
$c^{p}$ & Cost of a single paging & 0.1 \\
$c^{u}$ & Cost of a single TAU & 1 \\
$D$ & Number of data sets & 168 \\
$N$ & Number of sites & 60 \\
$T$ & Number of TAs & {$[11-30]$} \\
\hline
\end{tabular}

To apply SON on the standard TA scheme, the data is calculated according to the exponential filters explained in Section 10.1. This data is used for the standard TA design of each time interval. However in SON, the TA layout is applied to the network's data of the next time interval. This assumption 


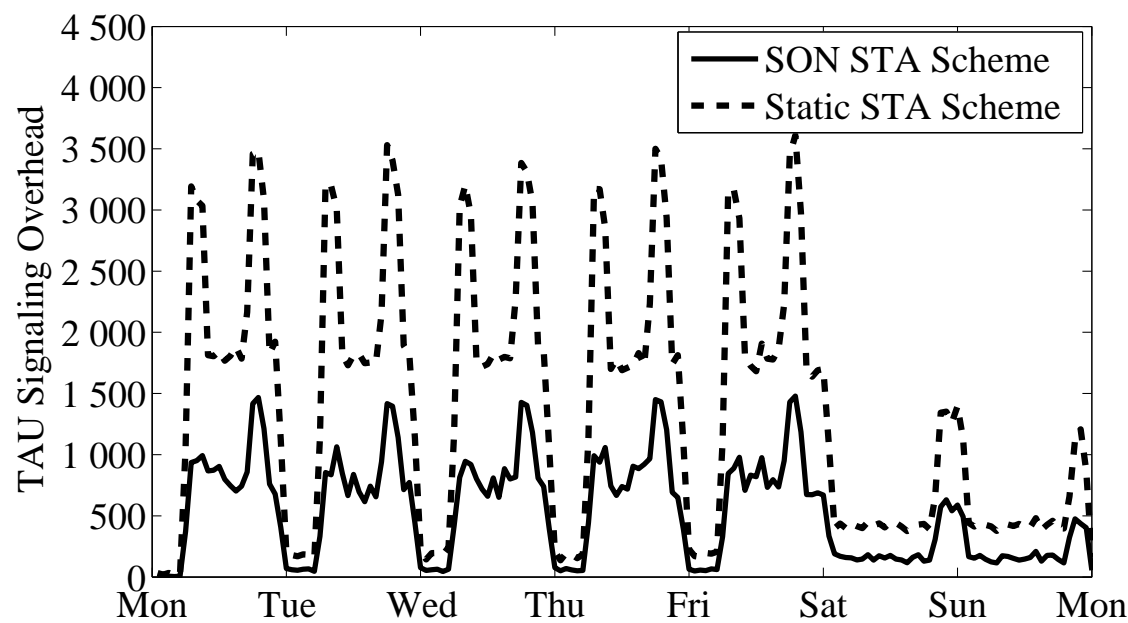

Figure 10.4: The TAU signaling overhead of the static and SON standard TA schemes.

has been previously explained in Section 10.1, and it is because in reality there is no possibility of going back in time and applying the optimum design to the same set of data. Note that the optimum design based on the history of UEs' behaviors might not perform well for the next time interval. The $\lambda$ value in this study is set to 0.75 , this means that the current time interval has three times more impact than the aggregated data of the previous time intervals. Larger values of $\lambda$ actually reduce the level of smoothing, and in case of $\lambda=1$ the data applied is just the same as the original data $u_{i}(d-1)$ and $h_{i j}(d-1)$ for calculating the actual signaling overhead. While values of $\lambda$ closer to zero have a greater smoothing effect and are less responsive to recent changes. The solid lines in Figures 10.3 and 10.4 illustrate the total and TAU signaling overhead of the SON standard TA scheme during the examined week.

Figure 10.3 compares the total signaling overhead of the static and SON standard TA scheme during the examined week. The key statistics of the TA design are as follows. There are 26 TAs in the static design, whereas in the SON designs the number of TAs in different time intervals varies from a minimum value of 11 to a maximum of 30 . The total signaling overhead of the static design during one week is $\sum_{d=1}^{D}\left(C_{\text {static }}^{U}(d)+C_{\text {static }}^{P}(d)\right)=2.6304 \times$ $10^{5}$, while in SON it has a $35.9 \%$ lower value, $\sum_{d=1}^{D}\left(C_{S O N}^{U}(d)+C_{S O N}^{P}(d)\right)=$ 


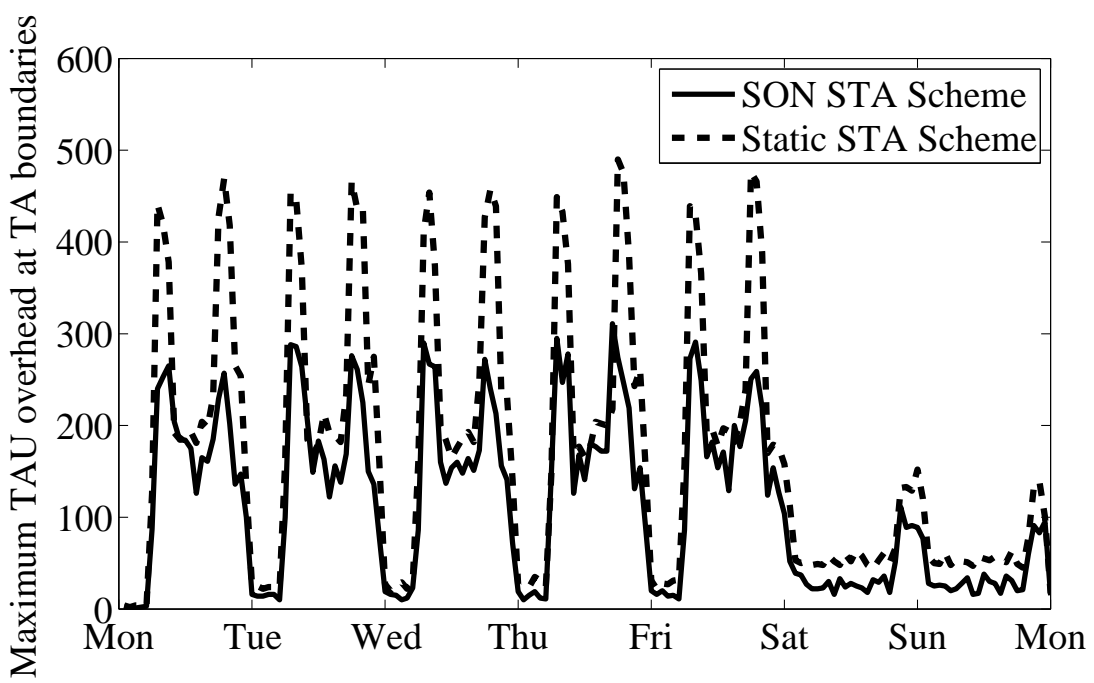

Figure 10.5: The maximum TAU signaling congestion of the static and SON standard TA schemes.

$1.6864 \times 10^{5}$. According to Figure 10.4 which compares the TAU overhead of the static and SON standard TA schemes during the examined week, it can be concluded that the improved total signaling overhead of the dynamic SON framework over the static one is a result of TAU overhead improvement. The total TAU overhead of the week is $\sum_{d=1}^{D} C_{\text {static }}^{U}(d)=2.3283 \times 10^{5}$ for the static TA, and $\sum_{d=1}^{D} C_{S O N}^{U}(d)=9.0235 \times 10^{4}$ for SON, that is $61.24 \%$ reduction.

The next step is to evaluate the problem of TAU congestion while a standard TA design based on the minimization of total signaling overhead is applied to the network. For the same static and SON frameworks explained above, the maximum and average TAU overhead have been computed at TA boundaries of the standard TA design for each time interval. Figure 10.5 presents the maximum value of TAU among all TA boundaries for both static (dashed line) and SON (solid line) frameworks. Although the figure shows that the static framework has higher TAU congested TAs compared to SON in the whole time frame, the difference is more highlighted for two peaks in the morning and afternoon of the working days.

Figure 10.6 provides an illustration of the average TAU overhead at TA boundaries for the two frameworks. This explains that the SON standard TA scheme outperforms the static TA mostly on reducing the average TAU overhead, however the problem of congested TAs still remains while compar- 


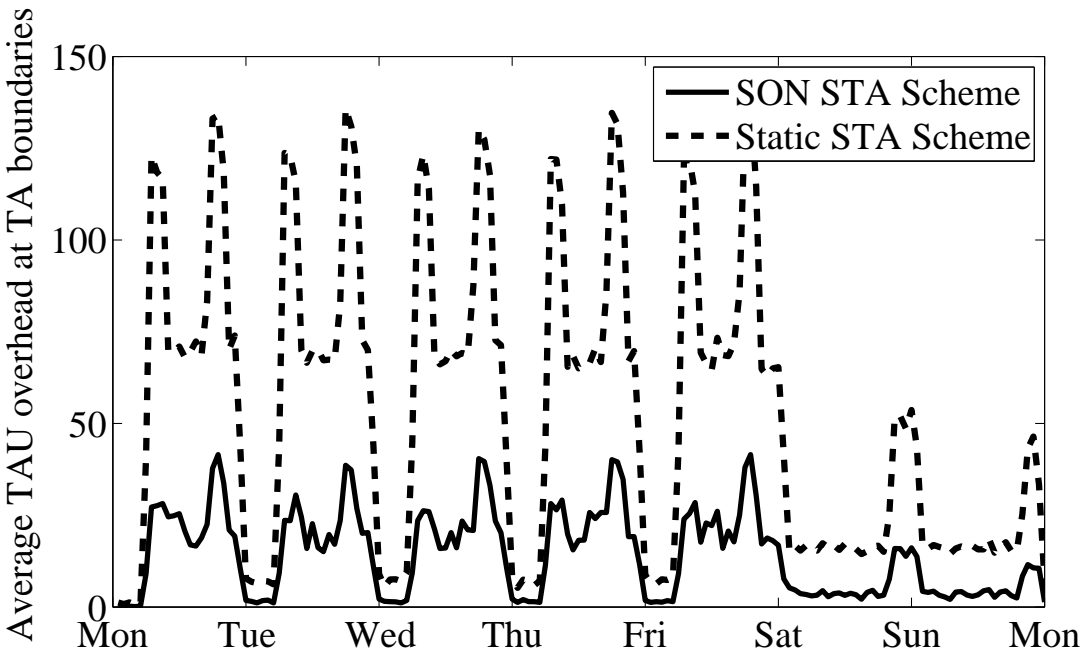

Figure 10.6: The average TAU signaling congestion of the static and SON standard TA schemes.

ing Figures 10.5 and 10.6. This motivates the idea of applying the proposed overlapping TAL scheme of Chapter 9 in order to mitigate the congestion situations.

\subsubsection{Applying the Overlapping TAL for Congestion Mitigation}

In this section, the aim is to mitigate the TAU congestion in the SON standard TA scheme presented with solid line in Figure 10.5. This figure shows that the problem of TAU congestion does not exist in all time intervals. The threshold value $\xi=100$ is set, and hence the overlapping TAL scheme is only applied to the data of time intervals in which the maximum TAU at the TA boundaries is higher than this threshold value.

Figure 10.7 compares the TAU congested scenario of the SON standard TA scheme with the uncongested scenario while the overlapping TAL scheme is applied to the network. For each time interval, it is assumed that $\beta=0.1$ in formulation (9.5), meaning that the model allows $10 \%$ increase in the total paging of each time interval in order to minimize the TAU congestion. The horizontal line at 100 shows which time intervals are congested, and hence the optimization model is applied only to those time intervals. The figure shows that with $\beta=0.1$ the maximum TAU remains well below the thresh- 


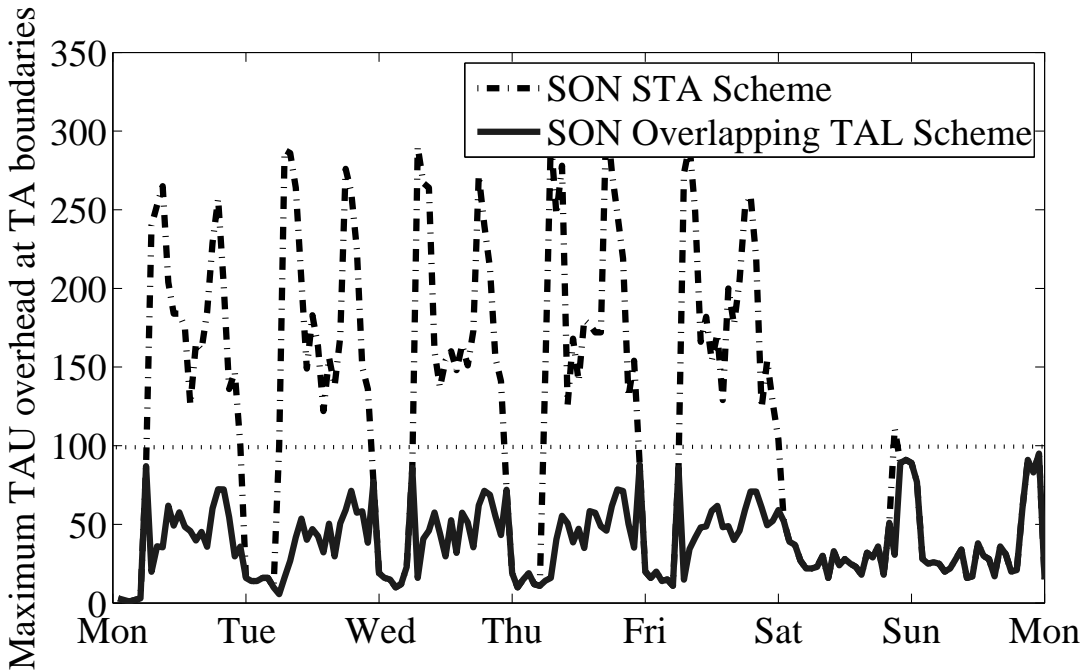

Figure 10.7: The TAU signaling congestion mitigation by the overlapping TAL scheme $(\beta=0.1)$.

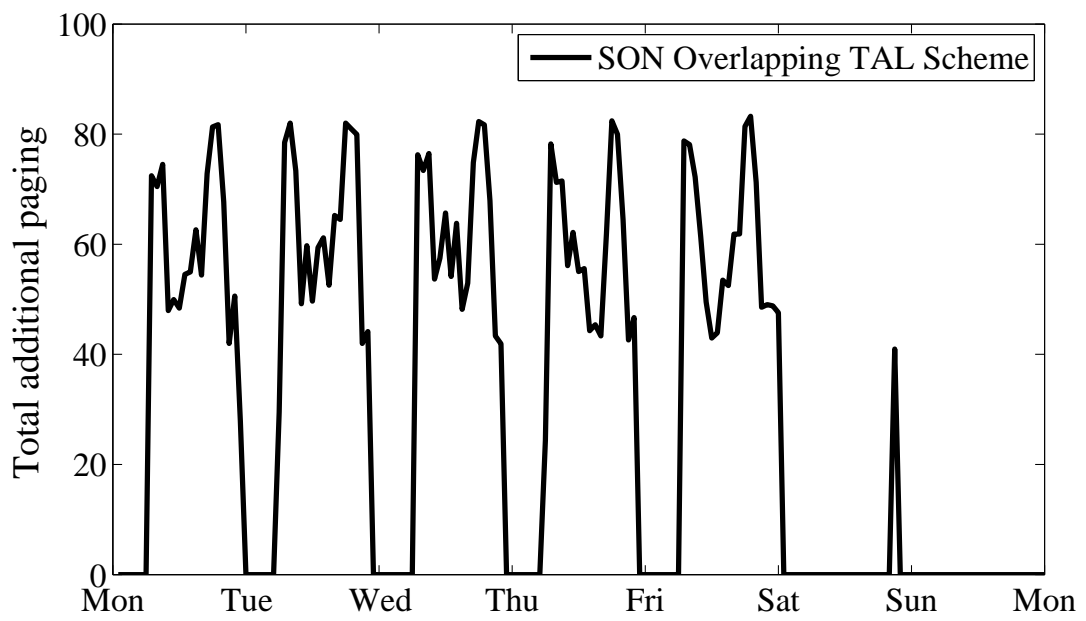

Figure 10.8: Total additional paging $(\beta=0.1)$.

old value $\xi=100$.

The goal is to avoid any TAU congestion however this does not mean to 


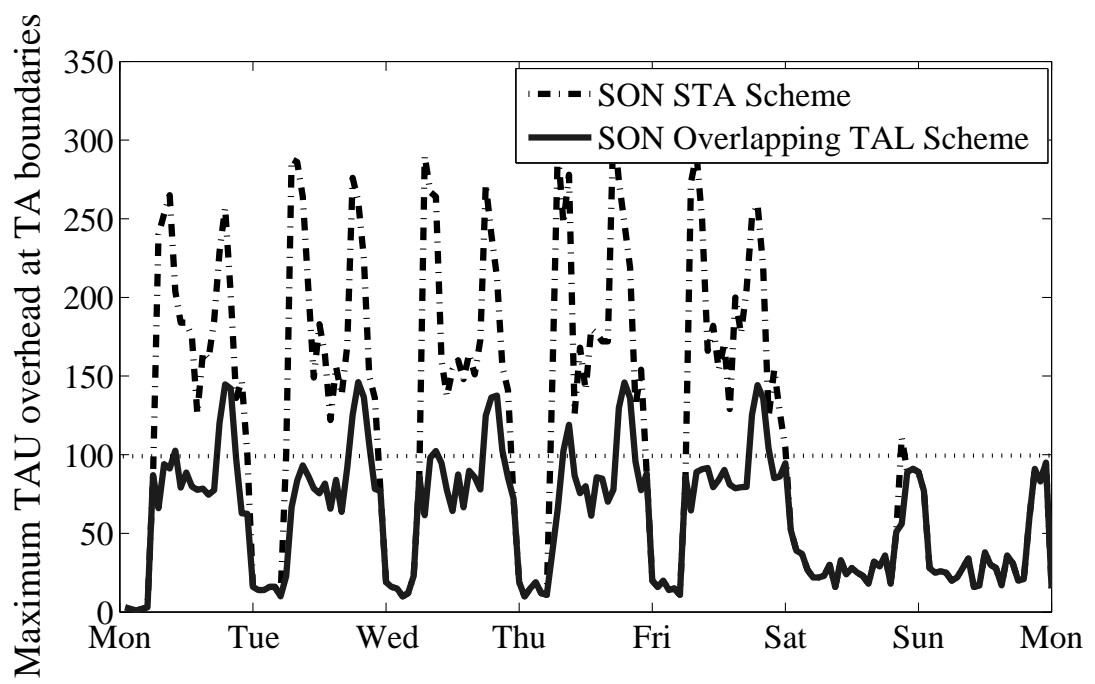

Figure 10.9: The TAU signaling congestion mitigation by the overlapping TAL scheme $(\beta=0.05)$.

reduce the TAU overhead as much as possible. Note that additional paging overhead is added in each time interval by introducing the overlapping TAL scheme. Figure 10.8 illustrates the amount of additional paging for each time interval for avoiding congestion. The total additional paging for the whole week is $\sum_{d=1}^{D} C_{\text {additional }}^{P}(d)=5.1947 \times 10^{3}$ for $\beta=0.1$.

In the final step of the performance evaluation, the value of $\beta$ is reduced to 0.05 in order to see if the overlapping TAL scheme is able to mitigate the TAU congestion. Figure 10.9 compares the TAU congested scenario of SON standard TA scheme with the SON overlapping TAL scheme while $\beta=0.05$. The figure shows that the overlapping TAL scheme is able to mitigate the TAU congestion problem in most of the time intervals, and for the time intervals which still have congestion, the maximum TAU overhead at the boundaries has been significantly reduced. Figure 10.10 illustrates that the total additional paging in the case where $\beta=0.05$ is only $\sum_{d=1}^{D} C_{\text {additional }}^{P}(d)=2.5973 \times 10^{3}$.

From the numerical results, it is concluded that the proposed overlapping TAL scheme is highly able to mitigate the TAU congestion in a SON dynamic framework. To fully mitigate the TAU congestion problem while not adding extra additional paging, SON can dynamically change the value of $\beta$ 


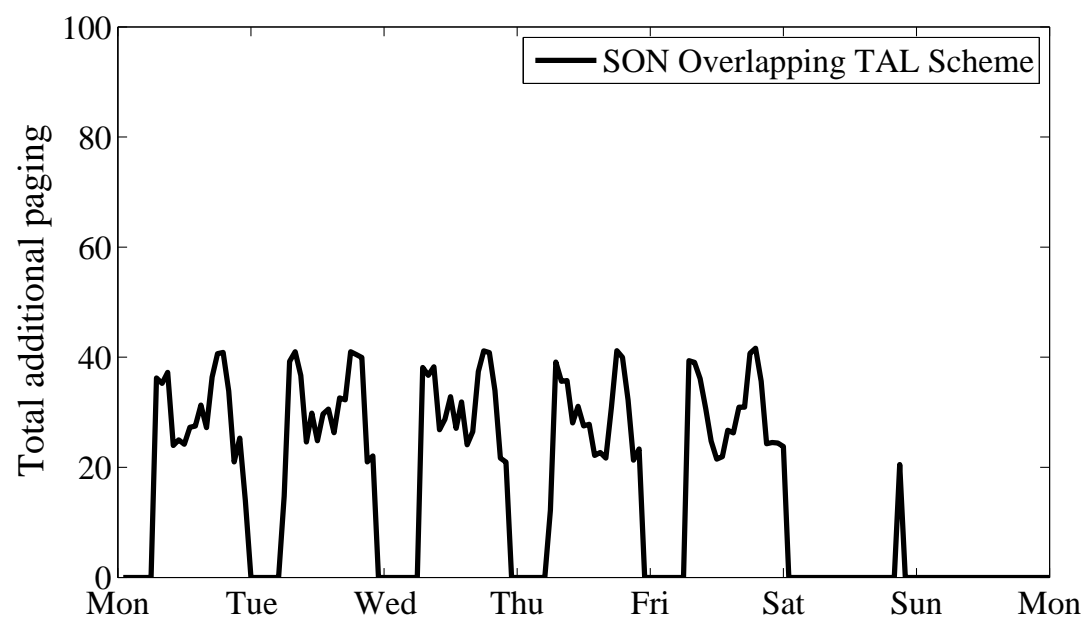

Figure 10.10: Total additional paging $(\beta=0.05)$.

based on the amount of congestion in different time intervals. Nevertheless, the performance of a fixed $\beta$ is evidently satisfactory.

\subsection{Conclusions}

In this chapter, an automated process was proposed in location management of LTE networks within the paradigm of self-organizing networks. The performance of the standard TA scheme was examined under time-vary data within the static and SON dynamic frameworks. The problem of mitigating TAU signaling congestion was tackled in a dynamic framework by using the overlapping TALs. The TAU and paging statistics have been monitored and the proportional use of TALs were adapted based on a linear program optimization formulation. Numerical evaluations for mitigating massive TAU demonstrated that the proposed dynamic optimization framework is very effective. The study was limited in having the data of only one week, however by introducing more history of network's behavior, SON can significantly improve the network performance. 



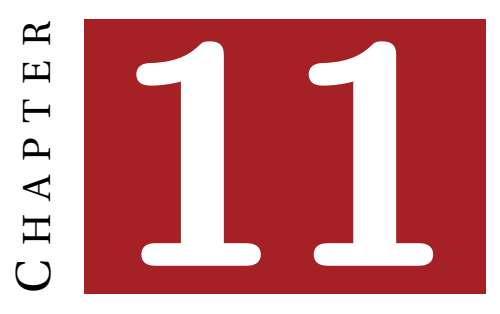

\section{Conclusions And Future Research}

The dissertation work has dealt with four main themes. The first theme (Chapters 3-4) was TA design re-optimization considering a cost budget, and finding the Pareto-optimal solutions for the trade-off between the signaling overhead and the reconfiguration cost. Although these problems were studied in the TA context, the results can be generalized to the study of LA and RA optimization. The second theme (Chapters 5-7) dealt with the TAL concept and its potentials compared to the standard TA scheme. There are still many open problems which require more investigation in the TAL scheme. The dissertation gave insight into the performance of TAL. The third theme (Chapters 8-9) dealt with the signaling congestion mitigation problem. The dissertation proposed an overlapping TAL scheme which solves the well-known train problem scenario, and can be generalized to mitigate the TAU and paging congestion in general topology. The fourth theme was the dynamic framework explored in Chapter 10. LTE supports SON, which is one of the visions in future network management. The dissertation examined the standard TA and the overlapping TAL schemes under a dynamic evaluation framework, in order to investigate the aptness of the schemes for SON.

\subsection{Conclusions}

The work presented in the dissertation justifies the benefit of tracking area planning and optimization for improving the performance in cellular networks. There are also some detailed conclusions from each specific theme studied in the dissertation. 
Once a TA design is in use, adopting a new solution of green-field optimization does not typically pay off in real networks. The repeated local search algorithm which is developed to solve the re-optimization problem in this dissertation is able to approach high-quality solutions. The novelty of the approach is the consideration of the reconfiguration-cost budget.

Before applying any reconfiguration, a decision maker can be provided by a set of Pareto-optimal solutions of potential trade-offs between the signaling overhead and the reconfiguration cost. The proposed integer programming model provides the exact Pareto-optimal solutions, and the suggested GA algorithm gives close to optimal solutions for large-scale networks in short time.

The signaling overhead obtained from the TAL assigned by the presented local-search algorithm is half of the signaling overhead resulted from the standard TA scheme. The rule of thumb in the dissertation is a very simple and quick approach for assigning a reasonably good TAL for a large-scale network.

Even if each cell is allowed to have a set of TALs to assign to its UEs, the optimum TAL solution based on minimizing the total signaling overhead is to consider only one TAL for each cell. This conclusion is obtained from the LP model, and justifies the previously studied approaches, such as the rule of thumb.

The proposed overlapping TAL scheme is able to successfully mitigate both TAU and paging signaling congestion. The scheme is invaluable due to its independence from each UE's specific mobility behavior.

The SON dynamic framework is able to significantly improve the performance of the network, even if a standard TA scheme is applied. The overlapping TAL scheme can be applied whenever a congestion scenario occurs. The study shows that the scheme is able to successfully mitigate the signaling congestion in a dynamic fashion.

\subsection{Suggestions for Future Work}

There are still many open problems in the study of TA management of cellular networks. Some related topics deserving further research are summarized below.

The re-optimization problem explored in the dissertation for the standard TA scheme can be extended to the TAL scheme. Even though in the TAL scheme the problem of service interruption in reconfiguration is solved, from the network standpoint it is still more preferable to avoid major changes 
between two consecutive configurations. Hence, another extension is to introduce a "change" budget in reconfiguring the TAL. It is also of interest to study the proposed TAL schemes under the defined SON dynamic framework.

In the performance evaluation of the static and dynamic frameworks, the aggregated data is based on short and equal-length time intervals. Due to the effort required for data collection, it is of relevance to evaluate the total signaling overheads which resulted from considering data with higher level of aggregation.

Additional experimental analysis on larger networks and various topologies can give more insights into the performance of the proposed algorithms. Another topic is the investigation of alternative and better optimization algorithms, especially for the TAL scheme, of which one example can be to extend the idea of rule of thumb to neighbors other than the first-hop ones in order to overcome the existing limitation.

One of the main extension lines of this research is to build an LM framework based on the overlapping TAL scheme. The framework should be compatible for both minimization of the total signaling overhead, and mitigation of the signaling congestion depending on the priority of either problem in the network. Reasonable thresholds must be computed for triggering the overlapping TAL assignments according to the two problems.

In order to offer wireless coverage in different environments, ranging from the open outdoors to office buildings and undergrounds, Heterogeneous Networks (HetNets) are defined [40]. HetNets are a gradual evolution of cellular topology that involve a mix of radio technologies and cell types (femto, pico, micro, and macro) working together seamlessly. HetNets consist of several subsystems, where each subsystem uses different access technologies and protocols [45]. Location management study of HetNets, in which LAs, RAs and TAs coexist, will introduce new research problems in this area. 



\section{BIBLIOGRAPHY}

[1] 3GPP. TS 23.002 - v9.1.0. Network architecture. 3G Release 9, 2009.

[2] 3GPP. TS 23.401 - v8.6.0. General Packet Radio Services (GPRS) enhancements for Evolved Universal Terrestrial Radio Access Network (EUTRAN) access. 3G Release 8, 2009.

[3] 3GPP. TR 23.880 - v0.1.0. Stage2 for registration in densely-populated area (RED). 3G Release 8, 2007.

[4] 3GPP. TS 23.882 - v1.9.0. System architecture evolution: Report on technical options and conclusions. 3G Release 7, 2007.

[5] 3GPP TS 24.301 - v12.1.0. Technical specification group core network and terminals; Non-Access-Stratum (NAS) protocol for Evolved Packet System (EPS). 3G Release 12, 2013.

[6] 3GPP TS 32.500 - v8.0.0. Self organizing networks (SON): concepts and requirements. 3G Release 8, 2008.

[7] 3GPP TS 36.300 - v8.0.0. Overall description; Stage 2. 3G Release 8, 2008.

[8] 3GPP TS 36.304 - v8.0.0. User Equipment (UE) procedure in idle mode. 3G Release 8, 2008.

[9] 3GPP TS 36.902. Evolved Universal Terrestrial Radio Access Network (E-UTRAN); Self-configuring and self-optimizing network (SON) use cases and solutions. 3G Release 9, 2009.

[10] 3G Americas. The benefits of SON in LTE. White Paper, 2009.

[11] E. H. L. Aarts and J. H. M. Korst. Simulated Annealing and Boltzmann Machines. Wiley, 1989.

[12] E. H. L. Aarts and J. K. Lenstra. Local Search in Combinatorial Optimization. Wiley, 1997.

[13] D. K. Agrafiotis. Multiobjective optimization of combinatorial libraries. IBM Journal of Research and Development, 2001. 
[14] N. Akkari, S. Tohme, and M. Doughan. Vertical-to-horizontal handovers: Architecture and mobility management. In Proc. of Wireless Communications and Networking Conference (WCNC'07), 2007.

[15] I. F. Akyildiz, J. S. M. Ho and Y. B. Lin. Movement-based location update and selective paging for PCS networks. ACM/IEEE Transactions on Networking, 4: 629-638, 1996.

[16] I. F. Akyildiz, J. McNair, J. S. M. Ho, H. Uzunalioglu, and W. Wang. Mobility management in next generation wireless systems. Proceedings of the IEEE, 87(8): 1347-1384, 1999.

[17] S. Z. Ali. Design of location areas for cellular mobile radio networks. In Proc. of IEEE Vehicular Technology Conference (VTC'02), pp. 1106-1110, 2002.

[18] S. Almeida-Luz, M. A. Vega-Rodrigeuz, J. A. Gomez-Pulido, and J. M. Sanchez-Perez. Applying different evolution to the reporting cells problem. In Proc. of IEEE International Multiconference on Computer Science and Information Technology, pp. 65-71, 2008.

[19] X. An, F. Pianese, I. Widjaja, and U. G. Acer. DMME: Virtualizing LTE mobility management. In Proc. of 36th IEEE Conference on Local Computer Networks, pp. 528-536, 2011.

[20] V. Angelakis, D. Gundlegård, B. Rajna, C. Rydergren, K. Vrotsou, R. Carlsson, J. Forgeat, and et al. Mobility modeling for transport efficiency - Analysis of travel characteristics based on mobile phone data. In Proc. of Mobile Phone Data For Development (D4D '13), 2013.

[21] C. Barnhart, E. L. Johnson, G. L. Nemhauser, M. W. P. Savelsbergh, and P. H. Vance. Branch-and-Price: Column generation for solving huge integer programs. Operations Research, 46(3): 316-329, 1998.

[22] A. Bar-Noy and I. Kessler. Tracking mobile users in wireless communications networks. IEEE Transactions on Information Theory, 39: 18771886, 1993.

[23] A. Bar-Noy, I. Kessler, and M. Sidi. Mobile users: To update or not to update?. Wireless Networks, 2 :175-185, 1995. 
[24] Y. Bejerano, M. A. Smith, J. Naor, and N. Immorlica. Efficient location area planning for personal communication systems. ACM/IEEE Transaction on Networking, 14: 438-450, 2006.

[25] R. E. Bellman. Dynamic Programming. Princeton University Press, 1957.

[26] D. Bertsimas and J. N. Tsitsiklis. Introduction to Linear Optimization. Athena Scientific, 1997.

[27] C. Bettstetter. Mobility modeling in wireless networks: Categorization, smooth movement, and border effects. ACM SIGMOBILE Mobile Computing and Communications Review, 5: 55-66, 2001.

[28] P. S. Bhattacharjee, D. Saha, A. Mukherjee, and M. Maitra. Location area planning for personal communication services networks. In Proc. of the 2nd ACM International Workshop on Modeling, Analysis and Simulation of Wireless and Mobile Systems (MSWiM '99), pp. 95-98, 1999.

[29] E. Cayirci and I. F. Akyildiz. Optimal location area design to minimize registration signaling traffic in wireless systems. IEEE Transactions on Mobile Computing, 2: 76-85, 2003.

[30] M. Canales, J. R. Gallego, A. H. Solana, and A. Valdovinos. An adaptive location management scheme for mobile broadband cellular systems. In Proc. of the 6th International Symposium on Wireless Communication Systems (ISWCS '09), pp. 333-337, 2009.

[31] K. Chander, D. Juneja, S. Iyengar, and S. Mukhopadhyay. An agentbased smart solution for vertical handover in 4G. International Journal of Engineering Science and Technology, 2010.

[32] A. Charnes and W. W. Cooper. Goal programming and multiple objective optimization, European Journal of Operational Research, 1: 39-45, 1977.

[33] K. -H. Chiang and N. Shenoy. A 2-D random-walk mobility model for location-management studies in wireless networks. IEEE Transactions on Vehicular Technology, 53: 413-424, 2004.

[34] M. I. Chowdhury, M. Iqbal, N. Sultana, and F. Rahman. Analysing an analytical solution model for simulations mobility. International Journal of Wireless \& Mobile Networks, 5: 111-124, 2013. 
[35] Y. W. Chung. Adaptive design of tracking area list in LTE. In Proc. of the 8th IEEE International Conference on Wireless and Optical Communications Networks (WOCN'11), 2011.

[36] D. Chung, H. Choo and H. Y. Youn. Reduction of location update traffic using virtual layer in PCS. In Proc. of the International Conference on Parallel Processing (ICPP '01), pp. 331-338, 2001.

[37] V. Chvatel. Linear Programming. Freeman, New York, 1983.

[38] W. J. Cook, W. H. Cunningham, W. R. Pulleyblank, and A. Schrijver. Combinatorial Optimization. Wiley, 1997.

[39] J. Cowling. Dynamic Location Management in Heterogeneous Cellular Networks, Thesis, University of Sydney, 2004.

[40] A. Damnjanovic, J. Montojo, Y. Wei, T. Ji, T. Luo, M. Vajapeyam, T. Yoo, O. Song, and D. Malladi. A survey on 3GPP heterogeneous networks. IEEE Wireless Communications, 18(3): 10-21, 2011.

[41] G. B. Dantzig. Linear Programming and Extensions. Princeton University Press, 1963.

[42] I. Demirkol, C. Ersoy, M. U. Caglayan, and H. Delic. Location area planning in cellular networks using simulated annealing. In Proc. of IEEE International Conference on Computer Communications (INFOCOM '01), pp. 13-20, 2001.

[43] I. Demirkol, C. Ersoy, M. U. Caglayan and H. Delic. Location area planning and cell-to-switch assignment in cellular networks. IEEE Transactions on Wireless Communications, 3: 880-890, 2004.

[44] M. Ehrgott and X. Gandibleux (editors). Multiple Criteria Optimization: State of the Art Annotated Bibliographic Surveys. Kluwer Academic Publishers, 2002.

[45] G. Fan, X. Lu, and S. Ci. Mobility Management in Heterogeneous Networks, in Handbook of Computer Networks: LANs, MANs, WANs, the Internet, and Global, Cellular, and Wireless Networks. John Wiley \& Sons, Inc., 2007.

[46] G. Fan, I. Stojmenovic, and J. Zhang. Elimination of generalized pingpong effects using triple-layers of location areas in cellular networks. Computer Science and Information Systems, 5: 1-16, 2008. 
[47] J. Ferragut and J. Mangues-Bafalluy. A self-organized tracking area list mechanism for large-scale networks of femtocells. In Proc. of International Conference on Communications (ICC'12), 2012.

[48] P. C. Fishburn. Lexicographic orders, utilities and decision rules: A survey. Management Science, 20: 1442-1471, 1974.

[49] P. Garcia, V. Casares, and J. Mataix. Reducing location update and paging costs in a PCS network. IEEE Transactions on Wireless Communications, 1: 200-209, 2002.

[50] M. R. Garey and D. S. Johnson. Computers and Intractability: A Guide to the Theory of NP-Completeness. W. H. Freeman and Company, 1979.

[51] F. Glover. Tabu search - part 1. ORSA Journal on Computing, 1(2): 190206, 1989.

[52] F. Glover. Tabu search - part 2. ORSA Journal on Computing, 2(1): 4-32, 1990.

[53] F. Glover and M. Laguna. Tabu Search. Kluwer Academic Publishers, 1997.

[54] D. E. Goldberg. Genetic Algorithms in Search, Optimization, and Machine Learning. Addison-Welsey, 1989.

[55] P. R. L. Gondim. Genetic algorithms and location area partitioning problem in cellular networks. In Proc. of IEEE Vehicular Technology Conference (VTC '96), pp. 1835-1838, 1996.

[56] Gurobi 3.0.1. Reference Manual. 2010.

[57] F. S. Hillier and G. J. Lieberman. Introduction to Mathematical Programming. McGraw-Hill, 1991.

[58] D. S. Hochbaum. Approximation Algorithms for NP-hard Problems. PWS Publishing, 1995.

[59] J. H. Holland. Adaptation in Natural and Artificial Systems. The University of Michigan Press, Ann Arbor, 1975.

[60] ILOG CPLEX 10.0. User’s Manual. 2006. 
[61] H. Isermann. Proper efficiency and the linear vector maximum problem. Operations Research, 22: 189-191, 1974.

[62] J. K. Karlof. Integer Programming: Theory and Practice. CRC Press, 2006.

[63] N. Karmarkar. A new polynomial time algorithm for linear programming. Combinatorica, 4(4): 373-395. 1984.

[64] J. K. Karlof, C. D. Gelatt, and M. P. Vecchi. Optimization by simulated annealing. Science, 220(4598): 671-680, 1983.

[65] J. R. Koza. Survey of genetic algorithms and genetic programming. In Proc. of WESCON '95 - Conference Record: Microelectronics, Communications Technology, Producing Quality Products, Mobile and Portable Power, Emerging Technologies, 1995.

[66] K. Kyamakya and K. Jobmann. Location management in cellular networks: Classification of the most important paradigms, realistic simulation framework, and relative performance analysis. IEEE Transactions on Vehicular Technology, 54: 687-708, 2005.

[67] A. H. Land and A. G. Doig. An automatic method for solving discrete programming problems. Econometrica, 28: 479-520, 1960.

[68] C. Y. Lee, S. J. Kim and T. Park. A design of multi-layered location registration areas in micro-cellular systems, Telecommunication Systems, 14: 107-120, 2000.

[69] B. Liang and Z. Haas. Predictive distance-based mobility management for PCS networks. In Proc. of IEEE Conference on Computer Communications (INFOCOM '99), pp. 1377-1384, 1999.

[70] Y. B. Lin. Reducing location update cost in a PCS network, IEEE Transactions on Networking, 5: 25-33, 1997.

[71] R. -H. Liou, Y. -B. Lin, and S. -C. Tsai. An investigation on LTE mobility management. IEEE Transactions on Mobile Computing, 12: 166-175, 2013.

[72] J. Lundgren, M. Rönnqvist, and P. Värbrand. Optimization. Studentlitteratur, 2010. 
[73] G. L. Lyberopoulos, J. G. Markoulidakis, D. V. Polymeros, D. F. Tsirkas, and E. D. Sykas. Intelligent paging strategies in future mobile telecommunication networks, IEEE Transactions on Vehicular Technology, 44: 534-554, 1995.

[74] T. Mach and R. Tafazolli. Mass mobility signaling congestion avoidance mechanism using randomized time distribution of cell reselections. In Proc. of International Conference on Telecommunications (ICT '09), pp. 238-242, 2009.

[75] S. Martello and P. Toth. Knapsack Problems: Algorithms and Computer Implementations. John Wiley \& Sons, 1990.

[76] S. Mishra and O. K. Tonguz. Most recent interaction area and speedbased intelligent paging in PCS. In Proc. of IEEE Vehicular Technology Conference (VTC '97), pp. 505-509, 1997.

[77] J. E. Mitchell. Handbook of Applied Optimization. Oxford University Press, 2002.

[78] Mitsubishi Electric. Tracking Areas Sizes and Tracking Area List Optimization. Technical Report in 3GPP TSG RAN WG3 Meeting, R3071931, 2007.

[79] S. Modarres Razavi and D. Yuan. Performance improvement of LTE tracking area design: a re-optimization approach. In Proc. of the 6th ACM International Workshop on Mobility Management and Wireless Access (MobiWac '08), pp. 77-84, 2008.

[80] S. Modarres Razavi, D. Yuan, F. Gunnarsson, and J. Moe. Optimizing the trade-off between signaling and reconfiguration: A novel bi-criteria solution approach for revising tracking area design. In Proc. of IEEE Vehicular Technology Conference (VTC'09), 2009.

[81] S. Modarres Razavi, D. Yuan, F. Gunnarsson, and J. Moe. Exploiting tracking area list for improving signaling overhead in LTE. In Proc. of IEEE Vehicular Technology Conference (VTC '10), 2010.

[82] S. Modarres Razavi, D. Yuan, F. Gunnarsson, and J. Moe. Dynamic tracking area list configuration and performance evaluation in LTE. In Proc. of Global Communications (GLOBECOM) Workshops, 2010. 
[83] S. Modarres Razavi. Tracking Area Planning in Cellular Networks, Thesis, Linköping University, 2011.

[84] S. Modarres Razavi and D. Yuan. Mitigating mobility signaling congestion in LTE by overlapping tracking area lists. In Proc. of the 14th ACM International Conference on Modeling, Analysis and Simulation of Wireless and Mobile Systems (MSWiM'11), pp. 285-292, 2011.

[85] S. Modarres Razavi, D. Yuan, F. Gunnarsson, and J. Moe. Performance and cost trade-off in tracking area reconfiguration: A Paretooptimization approach. Journal of Computer Networks, 56: 157-168, 2012.

[86] S. Modarres Razavi and D. Yuan. Mitigating signaling congestion in LTE location management by overlapping tracking area lists. Journal of Computer Communications, 35: 2227-2235, 2012.

[87] IST-2000-28088 Momentum project. http://momentum.zib.de.

[88] Motorola. Long Term Evolution (LTE): A Technical Overview. Technical White Paper, 2007.

[89] Z. Naor and H. Levy. Minimizing the wireless cost of tracking mobile users: an adaptive threshold scheme. In Proc. of IEEE International Conference on Computer Communications (INFOCOM '98), pp. 720729, 1998.

[90] G. L. Nemhauser and L. A. Wolsey. Integer and Combinatorial Optimization. Wiley, 1988.

[91] NEC. Tracking Area Concepts and Influence on Paging Load. Technical Report in 3GPP TSG RAN2 Meeting, R2-070655, 2007.

[92] C. H. Park and S. Soni. A simulated annealing based solution approach for the two-layered location registration and paging areas partitioning problem in cellular mobile networks. European Journal of Operational Research, 178: 579-594, 2007.

[93] K. E. Parsopoulos, D. K. Tasoulis, and M. N. Vrahatis. Multiobjective optimization using parallel vector evaluated particle swarm optimization. In Proc. of IASTED International Conference on Artificial Intelligence and Applications (AIA '04), pp. 823-828, 2004. 
[94] J. Plehn. The design of location areas in a GSM network. In Proc. of IEEE Vehicular Technology Conference (VTC '95), pp. 871-875, 1995.

[95] G. P. Pollini and C. L. I. A profile-based location strategy and its performance. IEEE Journal on Selected Areas in Communications. 15: 14151424, 1997.

[96] R. Prakash, Z. Haas, and M. Singhal. Load-balanced location management for cellular mobile systems using quorums and dynamic hashing. Wireless Networks, 7: 497-512, 2001.

[97] QUALCOMM Europe and T-Mobile. New SON User Case: Tracking Area Optimization. Technical Report in 3GPP TSG RAN WG3 Meeting, R3071594, 2007.

[98] C. R. Reeves and J. E. Rowe. Genetic Algorithms, Principles and Perspectives: A Guide to GA Theory. Kluwer, 2002.

[99] C. Rose and R. Yates. Minimizing the average cost of paging under delay constraints. Wireless Networks, 1: 211-219, 1995.

[100] D. Saha, P. S. Bhattacharjee, and A. Mukherjee. Time efficient heuristics for cell-to-switch assignment in quasi-static/dynamic location area planning of mobile cellular network. Computer Communications, 30: 326-340, 2007.

[101] S. K. Sen, A. Bhattacharya, and S. K. Das. A selective location update strategy for PCS users. Wireless Networks, 5: 313-326, 1999.

[102] I. Shayea, M. Ismail, and R. Nordin. Advanced handover techniques in LTE-advanced system. In Proc. of International Conference on Computer and Communication Engineering (ICCCE '12), 2012.

[103] G. Sierksma. Integer and Linear Programming: Theory and Practice. Marcel Dekker, 1996.

[104] R. E. Steuer. Multiple Criteria Optimization: Theory, Computation, and Application. John Wiley \& Sons, 1986.

[105] R. E. Steuer, L. R. Gardiner, and J. Gray. A bibliographic survey of the activities and international nature of multiple criteria decision making. Journal of Multi-Criteria Decision Analysis, 5: 195-217, 1996. 
[106] S. Subramaniam and G. Krishnamurthi. Load balancing location management. In Proc. of IEEE International Conference on Communications (ICC '01), pp. 2835-2839, 2001.

[107] S. Tabbane. An alternative strategy for location tracking. IEEE Journal on Selected Areas in Communications, 13: 880-892, 1995.

[108] E. G. Talbi. Metaheuristics: From Design to Implementation. John Wiley \& Sons, 2009.

[109] D. W. Tcha, T. J. Choi, and Y. S. Myung. Location-area partition in a cellular radio network. Journal of the Operational Research Society, 48: 1076-1081, 1997.

[110] Telecom Cloud. LTE TA planning, 31. 11/100 56-HSC 105 50/1 Uen B, 2009.

[111] G. Wan and E. Lin. A dynamic paging scheme for wireless communication systems. In Proc. of the 3rd ACM International Conference on Mobile Computing and Networking (MobiCom '97), pp. 195-203, 1997.

[112] W. Wang, I. F. Akyildiz, G. L. Stüber, and B. Chung. Effective paging schemes with delay bound as QoS constraints in wireless systems. Wireless Networks, 7: 455-466, 2001.

[113] W. L. Winston and M. Venkataramanan. Introduction to Mathematical Programming. Brooks/Cole - Thomson Learning, 2003.

[114] T. Winter. Mobility management and network design for UMTS. In Proc. of IEEE International Symposium on Personal, Indoor and Mobile Radio Communications (PIMRC '04), 2004.

[115] L. A. Wolsey. Integer Programming. Wiley, 1998.

[116] V. W. -S. Wong and V. C. M. Leung. Location management for next generation personal communication networks. IEEE Network, 14: 18-24, 2000.

[117] V. W. -S. Wong and V. C. M. Leung. An adaptive distance-based location update algorithm for PCS networks. In Proc. of IEEE International Conference on Communications (ICC '01), pp. 2001-2005, 2001. 
[118] Y. Zhang and M. Fujise. Location management congestion problem in wireless networks. IEEE Transactions on Vehicular Technology, 56: 942954, 2007.

[119] K. Zhu, D. Niyato, P. Wang, E. Hossain, and D. I. Kin. Mobility and handoff management in vehicular networks: A survey. Wireless Communications and Mobile Computing, 11(4): 459-476, 2011.

[120] E. Zitzler and S. Kunzli. Indicator-based selection in multi-objective search. In Proc. of the 8th International Conference on Parallel Solving from Nature (PPSN'04), pp. 832-842, 2004.

[121] ZTE. Self Optimization for Tracking Areas, Technical Report in 3GPP TSG RAN WG3 Meeting, R3-071415, 2007. 



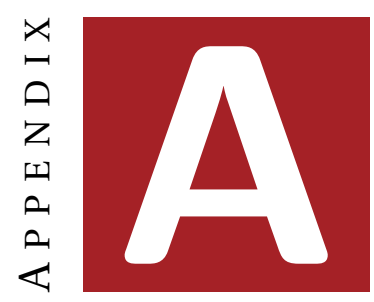

\section{Mathematical Models Notation}

\section{Parameters}

$\alpha \quad$ Call intensity factor (probability that a UE has to be paged)

$\beta \quad$ Allowed percentage increase on the total paging

$\gamma \quad$ Probability factor of UEs entering cell $i$ and have been a TAL in $Q_{i}$

$\delta \quad$ Probability factor of UEs in cell $i$ assigned to a TAL different from the one assigned by $i$

$\lambda \quad$ Smoothing factor

$\xi \quad$ Paging threshold value

$\sigma \quad$ Allowed percentage increase on the total TAU

$\tau \quad$ The time interval

$a_{i \ell} \quad$ Binary indicator that identifies if cell $i$ is in TAL $\ell$

$a_{\nu \ell} \quad$ Binary indicator that identifies if TA $v$ is in TAL $\ell$

$c^{p} \quad$ The overhead of a single paging

$c_{\text {paging }}(i) \quad$ The total paging of cell $i$

$c_{R}(\mathbf{t}) \quad$ The total cost of reconfiguration

$c_{S O}(\mathbf{t}) \quad$ The total signaling overhead of design $\mathbf{t}$

$c_{T A U}(i, j) \quad$ The total TAU overhead between $i$ and $j$

$c^{u} \quad$ The overhead of a single TAU

$\mathbf{d}\left(\mathbf{t}, \mathbf{t}^{0}\right) \quad$ Binary vector representing cells that have been assigned new TAs

$h_{i j} \quad$ Number of UEs having handover from cell $i$ to cell $j$

$n \quad$ Number of items in the knapsack problem

$n_{i} \quad$ length of a line segment starting with $i$ in the train scenario

$n_{v} \quad$ Number of cells in TA $v$ 


\section{Parameters (cont.)}

\begin{tabular}{ll}
$\mathbf{t}$ & Vector notation of cell-to-TA assignment \\
$\mathbf{t}^{0}$ & The TA design currently deployed \\
$t_{i}$ & The TA of cell $i$ \\
$u_{i}$ & Number of UEs in cell $i$ scaled by the time proportion spent in cell $i$ \\
$v_{i}$ & The value of item $i$ in the knapsack problem \\
$w_{i}$ & The weight of item $i$ in the knapsack problem \\
$z$ & Maximum TAU at TA boundaries or paging in a congestion scenario \\
$B$ & The budget value \\
$B^{\prime}$ & Total cell load factor corresponding to the budget level \\
$C^{P}$ & Total paging signaling overhead \\
$C^{P}(d)$ & Total paging signaling overhead of the data set $d$ \\
$C_{a d d i t i o n a l}^{P}$ & Total additional paging signaling overhead \\
$C_{M A X}^{P}$ & Maximum congestion in paging \\
$C^{U}$ & Total TAU signaling overhead \\
$C^{U}(d)$ & Total TAU signaling overhead in the data set $d$ \\
$C_{l}^{U}$ & Limit on additional increase in TAU \\
$C_{M A X}^{U}$ & Maximum congestion in TAU \\
$D$ & Number of data sets \\
$K$ & Number of iteration \\
$L_{t a l}$ & Length of the TAL \\
$M T A U$ & Maximum TAU \\
$N$ & Number of cells/sites \\
$N_{T A L}$ & Maximum number of TAs in TAL \\
POPSIZE & GA population size \\
PV & The Preference Value of the design \\
PV-MAX & The threshold on PV value \\
$\mathbf{S}(\mathbf{t})$ & Binary matrix presenting the TA configuration of all cells \\
$T$ & Number of TAs \\
$T P A G$ & Total additional paging \\
$W$ & Maximum weight that knapsack can carry \\
$X_{t a l}^{t a}$ & Percentage of UEs inside each $t a$ having tal as their list. \\
& \\
\hline &
\end{tabular}

Sets

$\mathscr{D} \quad$ Set of all data in a dynamic framework

$\mathscr{L} \quad$ Set of all possible TALs of $\mathscr{T}$

$\mathscr{N}$ Set of all cells/sites

$Q_{i} \quad$ Set of all neighbors of $i$ having $i$ in their TAL 


\section{Sets (cont.)}

$Q_{n i} \quad$ Set of neighbors of $n$ in $Q_{i}$ having both $i$ and $n$ in their TALs

$\mathscr{T}$ Set of TAs currently in use

\section{Variables}

$d_{i}\left(t, t^{0}\right) \quad$ Binary variable that identifies if cell $i$ has been assigned a new TA

$l_{i j} \quad$ Binary variable that identifies if cell $j$ is in the TAL of cell $i$

$p_{i t} \quad$ Binary variable that identifies if cell $i$ belongs to TA $t$

$p_{i t^{0}} \quad$ Binary variable that identifies if cell $i$ belongs to the current TA $t^{0}$

$s_{i j}(\mathbf{t}) \quad$ Binary variable that identifies if cell $i$ and $j$ are in the same TA

$s_{i j}^{d}(\mathbf{t}) \quad$ Binary variable that identifies if cell $i$ and $j$ are in the same TA in the data set $d$

$x_{i} \quad$ Binary variable that represents the knapsack problem

$x_{i j} \quad$ Continuous variable that represents the proportional use of TAL starting from $i$ to $j$ in a train scenario

$x_{i \ell} \quad$ Continuous variable that represents the proportional use of TAL $\ell$ by cell $i$

$x_{\ell} \quad$ Continuous variable that represents the proportional use of TAL $\ell$ by all cells of the TAs in $\ell$ 



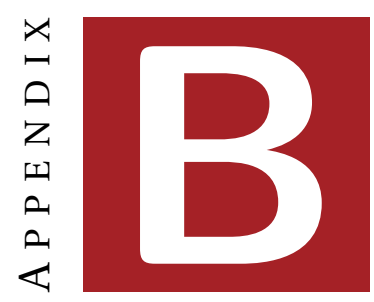

\section{UE-TRACES SCENARIO}

\section{B.1 Generating UE-traces Scenario}

Assuming that cell load, handover and the call intensity factor $(\alpha)$ are the available data of the UEs' mobility behaviors of a network in order to generate a reasonable UE-traces scenario, the following aspects should be considered.

- The UE is more probable to exist in cells with higher cell load. Equation (B.1), where $u_{i}$ is the load of cell $i$, estimates the probability ratio of cell $i$ being the starting cell of a UE in the specified time period.

$$
P_{\text {start }}(i)=\frac{u_{i}}{\sum_{j \in \mathscr{N}} u_{j}}
$$

- The UE tends to move towards neighbors with high handover value. Equation (B.2) gives the probability of a UE moving from cell $i$ to cell $j$, provided that the UE leaves cell $i$. The handover between $i$ and $j$ is defined by $h_{i j}$ and the set of neighbors of cell $i$ is denoted by $A_{i}$.

$$
P_{\text {move }}(i, j)=\frac{h_{i j}}{\sum_{p \in A_{i}} h_{i p}}
$$

- Among the UEs inside the UE-traces scenario, $\alpha$ of them are paged in the specified time period. 
In order to store a UE-traces scenario, a scenario matrix is defined. The number of UEs in the UE-traces scenario is denoted by $V$ and the time duration under investigation is denoted by $T$. Each row of the matrix represents one UE's movement during different time intervals of $T$. The serving cell of UE $v$ in time interval $\tau$ is stored as the element at $(\nu, \tau)$ of the scenario matrix. The length of each time interval is denoted by $\Delta \tau$, and the dimension of the scenario matrix is defined by $V \times \frac{T}{\Delta \tau}$. If the UE remains in the same cell for some time, the corresponding elements are identical in the intervals.

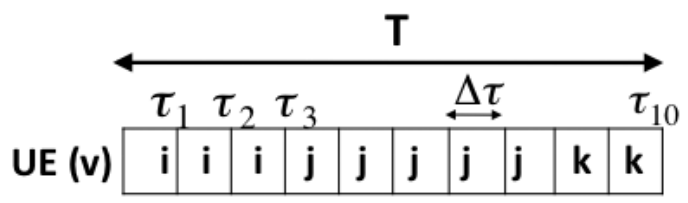

Figure B.1: An example of a row in the scenario matrix.

- Example: Figure B.1 illustrates an example of a row in the matrix. The time period $T$ is divided into ten equal time intervals $\left(\tau_{1}, \ldots, \tau_{10}\right)$. The UE stays in cell $i$ for $3 \Delta \tau$ and then it moves to cell $j$ and stays there for $5 \Delta \tau$. At $\tau_{8}$ the UE makes another move to cell $k$ and stays there for the rest of the time period.

The first column of the matrix are the starting cells of all UEs randomly generated based on Equation (B.1). The cell-to-cell movements of UEs are randomly generated according to (B.2). Based on the call intensity factor, $\lfloor\alpha V\rfloor$ UEs are randomly chosen to be paged in some intervals of $T$. The call duration for each paging is randomly chosen based on the Rayleigh distribution. During a call, the UE is active and the network knows the UE's location at cell level. Thus, there is no update overhead during a call.

\section{B.2 Aggregating Data from UE-traces Scenario}

Because the UE-traces scenario is generated based on the probabilities given by cell load and handover, the implied cell load and handover of the UEtraces scenario is not exactly the same as the original data. Thus, it is required to aggregate the cell load and handover from the UE-traces scenario.

The cell load $u_{i}$ is defined as the total number of UEs in cell $i$ scaled by the time proportions that the UEs spend in cell $i$. Therefore, the load of each 
cell in the network is aggregated by the scaled values of UEs staying in the cell using all the elements of the scenario matrix. The aggregated handover value is the number of moves from one cell to another.

- Example: Considering the example in Figure B.1 and that $\frac{\Delta \tau}{T}=0.1$, the aggregated cell load and handover from UE $v$ are:

$u_{i}=0.3, u_{j}=0.5, u_{k}=0.2, h_{i j}=1$, and $h_{j k}=1$. 
